\title{
THE CALTECH-NRAO STRIPE 82 SURVEY (CNSS) PAPER. I. THE PILOT RADIO TRANSIENT SURVEY IN $50 \mathrm{DEG}^{2}$
}

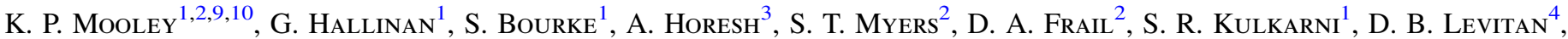

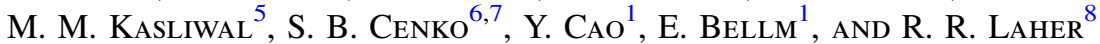 \\ ${ }^{1}$ Cahill Center for Astronomy, MC 249-17, California Institute of Technology, Pasadena, CA 91125, USA; kunal@astro.caltech.edu \\ ${ }^{2}$ National Radio Astronomy Observatory, P.O. Box O, Socorro, NM 87801, USA \\ ${ }^{3}$ Benoziyo Center for Astrophysics, Faculty of Physics, The Weizmann Institute for Science, Rehovot 76100, Israel \\ ${ }^{4}$ Microsoft, Bellevue, WA, USA \\ ${ }^{5}$ Carnegie Observatories, 813 Santa Barbara Street, Pasadena, CA 91101, USA \\ ${ }^{6}$ Astrophysics Science Division, NASA Goddard Space Flight Center, Mail Code 661, Greenbelt, MD 20771, USA \\ 7 Joint Space-Science Institute, University of Maryland, College Park, MD 20742, USA \\ ${ }^{8}$ Spitzer Science Center, California Institute of Technology, M/S 314-6, Pasadena, CA 91125, USA \\ Received 2015 April 29; accepted 2015 December 2; published 2016 February 11
}

\begin{abstract}
We have commenced a multiyear program, the Caltech-NRAO Stripe 82 Survey (CNSS), to search for radio transients with the Jansky VLA in the Sloan Digital Sky Survey Stripe 82 region. The CNSS will deliver five epochs over the entire $\sim 270 \mathrm{deg}^{2}$ of Stripe 82, an eventual deep combined map with an rms noise of $\sim 40 \mu \mathrm{Jy}$ and catalogs at a frequency of $3 \mathrm{GHz}$, and having a spatial resolution of $3^{\prime \prime}$. This first paper presents the results from an initial pilot survey of a $50 \mathrm{deg}^{2}$ region of Stripe 82, involving four epochs spanning logarithmic timescales between 1 week and $1.5 \mathrm{yr}$, with the combined map having a median rms noise of $35 \mu \mathrm{Jy}$. This pilot survey enabled the development of the hardware and software for rapid data processing, as well as transient detection and follow-up, necessary for the full $270 \mathrm{deg}^{2}$ survey. Data editing, calibration, imaging, source extraction, cataloging, and transient identification were completed in a semi-automated fashion within $6 \mathrm{hr}$ of completion of each epoch of observations, using dedicated computational hardware at the NRAO in Socorro and custom-developed data reduction and transient detection pipelines. Classification of variable and transient sources relied heavily on the wealth of multiwavelength legacy survey data in the Stripe 82 region, supplemented by repeated mapping of the region by the Palomar Transient Factory. A total of $3.9_{-0.9}^{+0.5 \%}$ of the few thousand detected point sources were found to vary by greater than $30 \%$, consistent with similar studies at 1.4 and $5 \mathrm{GHz}$. Multiwavelength photometric data and light curves suggest that the variability is mostly due to shock-induced flaring in the jets of active galactic nuclei (AGNs). Although this was only a pilot survey, we detected two bona fide transients, associated with an RS CVn binary and a dKe star. Comparison with existing legacy survey data (FIRST, VLA-Stripe 82) revealed additional highly variable and transient sources on timescales between 5 and $20 \mathrm{yr}$, largely associated with renewed AGN activity. The rates of such AGNs possibly imply episodes of enhanced accretion and jet activity occurring once every $\sim 40,000 \mathrm{yr}$ in these galaxies. We compile the revised radio transient rates and make recommendations for future transient surveys and joint radio-optical experiments.
\end{abstract}

Key words: catalogs - galaxies: active - radio continuum: galaxies - stars: activity - supernovae: general - surveys

\section{INTRODUCTION}

Owing to rapidly advancing detector technology and faster computing speeds, optical and high-energy astronomy have enjoyed a synoptic survey capability ${ }^{11}$ for over a decade. A rich discovery phase space has been revealed through synoptic experiments such as the Fermi Gamma-Ray Satellite (Michelson et al. 2010), the Swift Gamma-Ray Burst Alert Mission (Gehrels et al. 2009), and optical imagers such as the Catalina Real-Time Transient Survey, the Panoramic Survey Telescope Rapid Response System (Pan-Starrs), and the Palomar Transient Factory (PTF; Kasliwal 2012).

A similar science yield likely awaits centimeter radio surveys. In Table 1 we give a summary of known extragalactic and Galactic slow transients (timescale $>1 \mathrm{~s}$ ) at $\mathrm{GHz}$

\footnotetext{
${ }^{9}$ NRAO Grote Reber Fellow.

${ }^{10}$ Current address: Oxford Centre for Astrophysical Surveys, Denys Wilkinson Building, Keble Road, Oxford OX1 3RH.

${ }^{11}$ We refer to a "synoptic survey" here as a blind survey (as opposed to a targeted survey) sampling a large part of the sky (tens of square degrees or more) with a cadence of days to weeks.
}

frequencies (see also Murphy et al. 2013; Lazio et al. 2014). These known sources highlight that radio observations trace high-energy particles, arise in locations of high magnetic field, and probe the interaction of fast outflows with the surrounding medium. They are complementary to optical and high energies in that they are not affected by extinction and the emission from fast outflows is often not as narrowly beamed, providing reliable calorimetry and the true rates of cosmic explosions.

In recognition of these discovery opportunities, a new generation of centimeter-wavelength facilities have been built or are under way. These include the Karl G. Jansky Array (Jansky VLA; Perley et al. 2011), ASKAP (Johnston et al. 2008), MeerKAT (Booth \& Jonas 2012), and Apertif/WSRT (Oosterloo et al. 2010). The upgraded Jansky VLA is the first operational interferometer with the survey speed to routinely detect the extragalactic explosive population with modest time allocation. Unlike the legacy VLA, the Jansky VLA possesses frequency agility essential to characterize the broadband radio spectra of detected transients. It has a much improved snapshot multifrequency UV coverage; imaging artifacts and contamination by sidelobes (Bower et al. 2007; Frail et al. 2012) are no 
Table 1

Summary of Slow Radio Transient Phenomena at 1-6 GHz Frequencies

\begin{tabular}{|c|c|c|c|c|c|c|c|c|c|}
\hline \multirow[t]{2}{*}{ No } & \multirow[t]{2}{*}{ Object } & \multirow{2}{*}{$\begin{array}{l}\text { Variability } \\
\text { Process }\end{array}$} & \multirow{2}{*}{ Location } & \multicolumn{2}{|c|}{ Timescale } & \multicolumn{2}{|c|}{ Peak Luminosity } & \multirow{2}{*}{$\begin{array}{c}\text { Rate } \\
(>0.3 \mathrm{mJy}) \\
\left(\mathrm{deg}^{-2}\right)\end{array}$} & \multirow[t]{2}{*}{ References } \\
\hline & & & & Radio & Optical & $\begin{array}{c}\text { Radio } \\
\left(\mathrm{erg} \mathrm{s}^{-1} \mathrm{~Hz}^{-1} \text { ) }\right.\end{array}$ & $\begin{array}{c}\text { Optical } \\
\left(\mathrm{erg} \mathrm{s}^{-1}\right)\end{array}$ & & \\
\hline \multicolumn{10}{|c|}{ Extragalactic } \\
\hline 1 & AGN & (a) Quiescent & Nuclear & years-decades & years & $10^{27}-10^{34}$ & $10^{42}-10^{47}$ & 0.6 & $1-7$ \\
\hline & & (b) Shock-in-jet & $"$ & days-years & $\ldots$ & $10^{27}-10^{34}$ & $\ldots$ & 1.25 & $6-10$ \\
\hline & & (c) ISS & $"$ & minutes-days & $\ldots$ & $\ldots$ & $\ldots$ & 60 & $11-13$ \\
\hline & & (d) ESE & $"$ & months & $\ldots$ & $\ldots$ & $\ldots$ & 0.001 & $14-15$ \\
\hline & & (e) Jet-precession & $"$ & years & $\ldots$ & $\ldots$ & $\ldots$ & unknown & $16-17$ \\
\hline 2 & $\mathrm{SN}-\mathrm{II} / \mathrm{Ib} / \mathrm{c}$ & Shocked CSM & Traces SF & months-decades & days-months & $10^{25}-10^{29}$ & $10^{41}-10^{43}$ & $\begin{array}{c}0.04(\mathrm{II}), \\
6 \times 10^{-6}(\mathrm{Ib} / \mathrm{c})\end{array}$ & $18-23$ \\
\hline 3 & SN-Ia & Shocked CSM & Non-nuclear & days-weeks & days-months & $\lesssim 10^{23}$ & $10^{39}-10^{43}$ & $<10^{-5}$ & $23-28$ \\
\hline \multirow[t]{2}{*}{4} & Long-GRB & Shocked CSM/Jet & Traces SF & days-years & days-months & $10^{29}-10^{31}$ & $10^{43}-10^{47}$ & $6 \times 10^{-5}$ & 29 \\
\hline & $\mathrm{OA}$ & Shocked CSM & Traces SF & weeks-years & days? & $10^{29}-10^{30}$ & $10^{43}-10^{46}$ & $7 \times 10^{-4}$ & $30-32$ \\
\hline \multirow[t]{2}{*}{5} & Short-GRB & Shocked CSM/Jet & Non-nuclear & days-years? & days & $\sim 10^{25}$ & $10^{39} \sim 10^{41}$ & $<10^{-6}$ & $33-35$ \\
\hline & BNS merger & Shocked CSM & Non-nuclear & weeks-yr & days & $10^{22} \sim 10^{25}$ & $10^{39} \sim 10^{40}$ & $2 \times 10^{-4}$ & $31,36-37$ \\
\hline \multirow[t]{2}{*}{6} & TDE & Shocked CNM/Jet & Nuclear & yr? & months-yr? & $10^{30} \sim 10^{31}$ & $10^{42} \sim 10^{44}$ & 0.005 & $31,38-40$ \\
\hline & Off-axis TDE & Shocked CNM & Nuclear & yr? & unknown & $\sim 10^{30}$ & unknown & 0.01 & 31 \\
\hline 7 & AIC & Shocked CSM & Non-nuclear & months & day & unknown & $\sim 10^{39}$ & $6 \times 10^{-5}$ & $70-72$ \\
\hline \multicolumn{10}{|c|}{ Galactic } \\
\hline 1 & Active star & Flaring (magnetic) & Isotropic & hours-days & seconds-hours & $10^{12}-10^{15}$ & $10^{30} \sim 10^{33}$ & 0.02 & $7,41-44$ \\
\hline 2 & Active binary & Flaring (magnetic) & Isotropic & hours-days & hours-days & $10^{14}-10^{22}$ & $<10^{33} \sim 10^{34}$ & 0.01 & $7,41,43,46$ \\
\hline 3 & $\mathrm{X}$-ray binary & Mass accretion/flare & GP & days-weeks & minutes-hours & $10^{20} \sim 10^{21}$ & $\sim 10^{35}$ & 0.005 & $7,44,46-50$ \\
\hline 4 & $\mathrm{CV}$ & Dwarf nova/jet & GP & hours-days & days-weeks & $10^{16} \sim 10^{17}$ & $10^{33} \sim 10^{35}$ & 0.001 & $44,51-54$ \\
\hline 5 & YSO & Mass accretion/flare & GP & hours-weeks & hours-weeks & $10^{16}-10^{18}$ & $10^{31} \sim 10^{34}$ & $<0.05$ & $7,41,55$ \\
\hline 6 & Pulsar & Scattering & GP & weeks-months & $\ldots$ & $10^{15} \sim 10^{17}$ & $\ldots$ & 0.003 & $44,56,57$ \\
\hline 7 & Magnetar & Flaring (magnetic) & GP & weeks & unknown & $\sim 10^{20} ?$ & unknown & $<0.05$ & $7,58-60$ \\
\hline 8 & Brown Dwarf & Pulsing (magnetic) & Isotropic & seconds-hours & seconds-hours & $10^{13} \sim 10^{14}$ & $\sim 10^{24}$ & $<0.05$ & $7,61,62$ \\
\hline 9 & Novae & Ejecta/Shocked CSM & GP & $\mathrm{yr}$ & days-months & $\sim 10^{20}$ & $10^{38}-10^{40}$ & $<0.05$ & $23,63-65$ \\
\hline 10 & GCRT & Unknown & GP? & minutes-yr & unknown & unknown & unknown & unknown & 66-69 \\
\hline
\end{tabular}

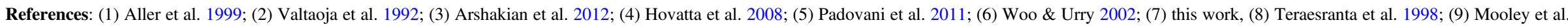

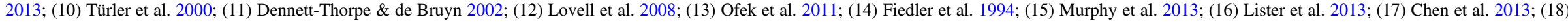

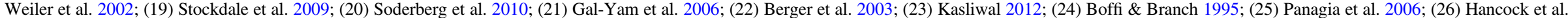

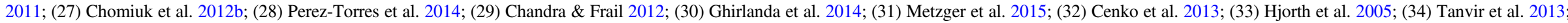

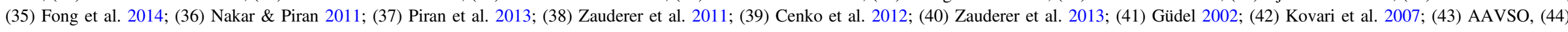

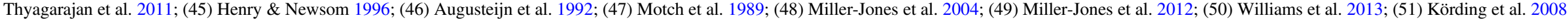

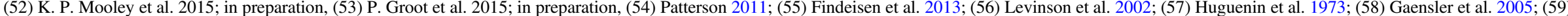

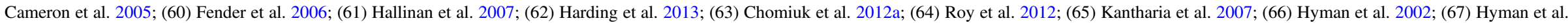
2005; (68) Hyman et al. 2007; (69) Hyman et al. 2009; (70) Piro \& Kulkarni 2013; (71) Metzger et al. 2009; (72) Darbha et al. 2010.

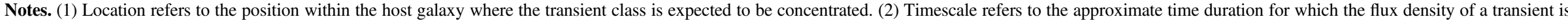

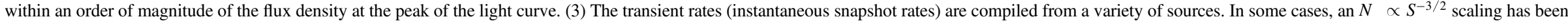

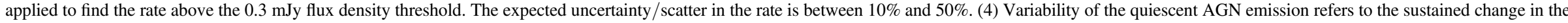

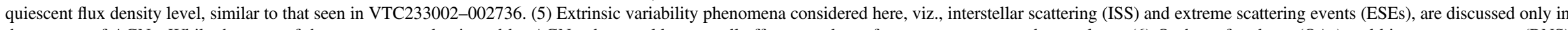

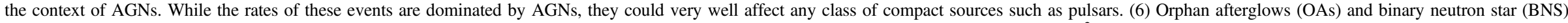

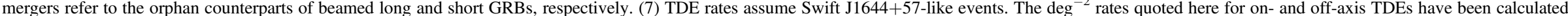

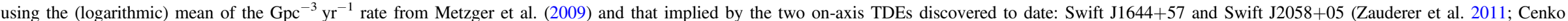
et al. 2012). 
longer major impediments in transient search. Furthermore, unlike the SKA pathfinders, the Jansky VLA has the spatial resolution to allow the accurate localization within a host galaxy (nuclear vs. non-nuclear) necessary to rule out the active galactic nucleus (AGN) activity that dominates the extragalactic transient and variable population.

To date, there have been rather few radio surveys dedicated to slow variables and transients, ${ }^{12}$ and they all have a number of limitations. The majority of these surveys were single, multiepoch interferometric pointings with a limited field of view, and as a result the number of variables and transients is low (e.g., Carilli et al. 2003; Mooley et al. 2013). Existing wide-area surveys are based on either archival data or the data reduction, and candidate source identification was carried out significantly delayed from the observing dates. This approach has drawbacks since without near-real-time data reduction and multiwavelength follow-up, the candidate lists contain ambiguous transient classifications (Bannister et al. 2011a, 2011b; Thyagarajan et al. 2011).

We note that wide-field, shallow surveys are superior to narrow field, deep surveys, since they have the advantage of bringing the detectable population of transients closer in distance, thus improving the ability to find optical/infrared counterparts and to characterize host galaxies and/or progenitors. In the Appendix we show mathematically that wide and shallow surveys are ideal for radio transient searches.

In the light of these factors and to address some of the limitations of past surveys, we have commenced a multiyear program, the Caltech-NRAO Stripe 82 Survey (CNSS), to search for slow radio transients with the Jansky VLA at $3 \mathrm{GHz}$ in the Sloan Digital Sky Survey Stripe 82 region. The CNSS is a dedicated transient survey carried out in five epochs (cadence of days, months, and years) over the entire $\sim 270 \mathrm{deg}^{2}$ of Stripe 82 , with a uniform single-epoch rms noise of $\sim 80 \mu \mathrm{Jy}$ and a spatial resolution of $\sim 3^{\prime \prime}$.

In this paper we present the CNSS pilot survey, a sub-mJy Jansky VLA survey at $3 \mathrm{GHz}$ in $50 \mathrm{deg}^{2}$ of Stripe 82 . This is a prototype survey to demonstrate the fast imaging capabilities of the VLA and to develop near-real-time data processing, source identification, and transient search. Another unique aspect of this radio survey is that it was undertaken with a contemporaneous high-cadence optical survey with the PTF so that a direct comparison could be made of the dynamic radio and optical skies. Technical details of the radio and the optical surveys are given in Section 2. The calibration, RFI flagging, imaging, and source cataloging of the Jansky VLA data carried out in nearreal-time, as well as during the final careful processing, is described in Section 3. Section 4 details the optical data processing for the contemporaneous survey with the PTF. A description of the radio transient search on timescales less than 1 week, 1 month, $1.5 \mathrm{yr}$, and longer timescales is provided in Section 5. A subset of the radio variable and transient sources representative of the full sample found in the pilot radio survey are discussed in detail in Section 5. The optical counterparts of radio sources from the Jansky VLA survey, as well as the optical variability of the radio transients from Section 5, are studied in Section 6 using photometry from PTF and SDSS. Section 6 also gives a brief discussion of the optical transients found in PTF independently of the radio survey. A summary of

\footnotetext{
12 http://www.tauceti.caltech.edu/kunal/radio-transient-surveys/index.html
}

the results from our joint radio-optical experiment and their implications are discussed in Section 7.

\section{THE SURVEY}

\subsection{Radio Observations}

The CNSS was designed to (1) logarithmically sample timescales roughly between 1 week and $1 \mathrm{yr}$, (2) have a high survey speed (which is a function of the bandwidth and the antenna system temperature), (3) have a relatively high angular resolution to facilitate a precise location of transients, (4) be carried out in a part of the sky where ample multiwavelength archival data were available so as to enable the identification of host galaxies or progenitors of the radio transients, (5) find nonthermal transients relatively early on in their evolution, and (6) be wide and sensitive enough to potentially detect or place strong constraints on binary neutron star (BNS) mergers and orphan long-duration gamma-ray burst afterglows (OAs).

For the pilot survey, we chose a $\sim 50 \mathrm{deg}^{2}$ region in SDSS Stripe 82 with similar characteristics. The region had to satisfy other scheduling constraints in the radio and optical, so we specifically chose the region bounded in R.A. by $329^{\circ} .127$ and $353^{\circ} .158$ and in decl. by $-1^{\circ} .132$ and $+1^{\circ} .167$, approximately. Observations were carried out across four epochs with the Jansky VLA in B array configuration, and S band was chosen to maximize survey speed. To maximize the continuum imaging sensitivity, the observing setup chosen was the Wideband Interferometric Digital Architecture (WIDAR) correlator with 16 spectral windows, $642 \mathrm{MHz}$-wide channels each to get $2 \mathrm{GHz}$ of total bandwidth centered on $3.0 \mathrm{GHz}$, and $1 \mathrm{~s}$ integrations.

The first three epochs were observed under the project code 12A-371 between 2012 July and August. Owing to telescope scheduling constraints, each of these epochs was divided into two nights of observing of $7 \mathrm{hr}$ each. All six $7 \mathrm{hr}$ observing blocks were centered on $23 \mathrm{hr}$ local sidereal time (LST). We refer to the three epochs as E1/2/3 and the regions observed on the two nights of the first epoch as R1 and R2, respectively. Observations were carried out at the same LST in the three epochs in order to minimize systematic effects associated with sidelobes and beam squint. Each of the regions, $\mathrm{R} 1$ and $\mathrm{R} 2$, was divided into 485 pointings arranged in a hexagonal mosaic pattern (Figure 1), optimized using the makeschedule task in CASA written by Andreas Brunthaler. In order to maximize the volume probed by our survey, we compromised on uniform-sensitivity coverage, placing neighboring pointings at $\geqslant 15^{\prime}$. The mosaic is therefore not precisely hexagonal close packed. ${ }^{13}$ Nearest neighbors having the same decl. are separated by $15^{\prime}$, and those having offset in decl. are $\sqrt{15^{\prime 2}+7 ! 5^{2}}=16^{\prime} .8$ apart. During each night of observing, one 39 s snapshot observation was obtained at each of the 485 target pointings. J2212+0152 and J2323-0317 were chosen as the phase calibrators for the two regions, respectively, and 3C 48 as the flux and bandpass calibrator. The phase calibrator was observed every 15 minutes. A summary of the first three observing epochs is given in Table 2.

The fourth epoch (E4) was observed under the project code $13 \mathrm{~B}-370$ and is essentially a co-addition of three epochs of observation carried out between 2013 November and 2014

\footnotetext{
13 Although epochs E1-E3 do not have uniform sensitivity across the survey region, the final CNSS survey is designed to have uniform rms noise.
} 


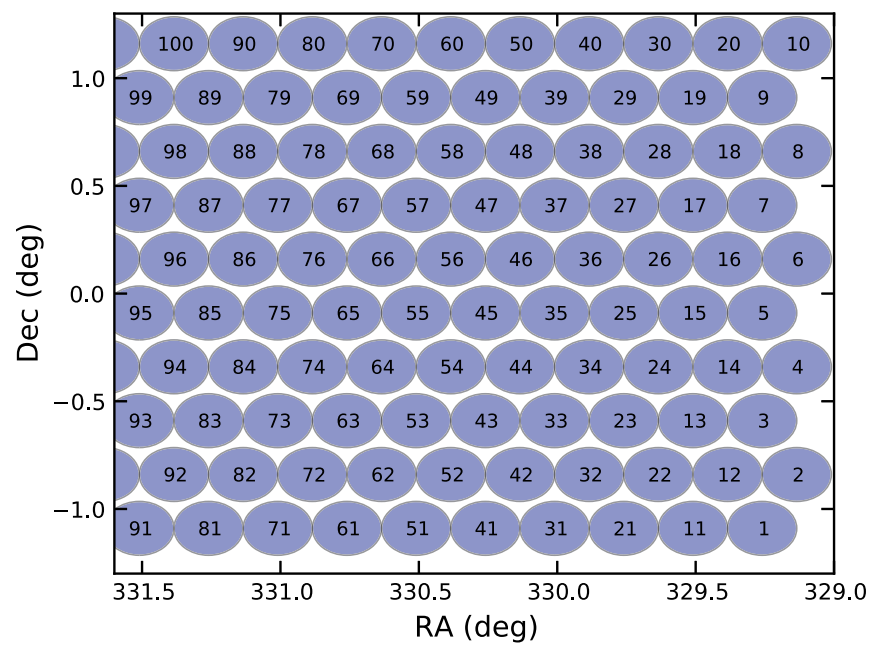

Figure 1. Setup of target pointings for a part of region R1 of the Jansky VLA survey. The circles indicate primary beam FWHM at the mean frequency of $2.9 \mathrm{GHz}$, and the pointing numbers are labeled. Nearest neighbors are separated by $15^{\prime}$. See Section 2.1 for details on the pointing setup.

February. For these observations, we took advantage of On-theFly mosaicing (OTF), ${ }^{14}$ available as a Resident Shared Risk Observing capability of the Jansky VLA. In the OTF mode, data were taken while the telescopes were slewing at a speed of $1^{\prime}$ per second along R.A., and the correlator phase center was stepped every $4 \mathrm{~s}$. In each observing block, $9^{\circ}$ in R.A. and $2^{\circ} .5$ in decl. centered on $0^{\circ}$ were observed to get a total areal coverage of $22.5 \mathrm{deg}^{2}$. Observations were carried out with the telescopes moving in a zig-zag basket-weave pattern on the sky: slewing $9^{\circ}$ in decreasing R.A. along a constant decl. strip at -1.25 , then stepping up in decl. by $10^{\circ} .6$ and slewing along increasing R.A., and so on. Covering 2.5 along decl. required 15 such zig-zag strips offset by $10{ }^{\prime} 6$ in decl. from nearest neighbors. This observing plan is designed to yield $20254 \mathrm{~s}$ long scans and a mosaic with a uniform rms noise of $\sim 80 \mu \mathrm{Jy}$ across $22.5 \mathrm{deg}^{2}$. Since these observations were carried out as part of a proposal to observe $270 \mathrm{deg}^{2}$ of SDSS Stripe 82, the details will be provided in a later publication (K. P. Mooley et al. 2015, in preparation). In this paper, we will be interested only in the region bounded in R.A. by $329^{\circ} .127$ and $353^{\circ} .158$ and in decl. by $-1^{\circ} .132$ and $+1^{\circ} .167$. A summary of the fourth epoch of observations is given in Table 2 .

\subsection{Optical Observations}

The optical survey, designed to be contemporaneous with the radio survey, was carried out with the $1.2 \mathrm{~m}$ (48-inch) Samuel Oschin Telescope at the Palomar Observatory as part of the PTF (Rahmer et al. 2008; Law et al. 2009; Rau et al. 2009). PTF uses a large field camera (CFH12k) consisting of a $6 \times 2$ array of $2048 \times 4096$ pixels $^{2}$ CCDs out of which one CCD is inactive. The camera subtends $\sim 7.2 \mathrm{deg}^{2}$ on the sky. The $50 \mathrm{deg}^{2}$ survey region was covered in 14 pointings, with each pointing overlapping by about $50 \%$ with its adjacent one (necessitated by one inactive CCD). The footprints of the 14 pointings (fields) are shown in Figure 2.

The PTF observations of the $50 \mathrm{deg}^{2}$ region were carried out between 2012 June 25 and September 25. The survey was done primarily in the $R$ band, but $g$-band observations are available

\footnotetext{
${ }^{14}$ https://science.nrao.edu/facilities/vla/docs/manuals/opt/otf
}

for some of the nights. The Stripe 82 fields were dynamically queued with the other PTF program fields, such that the frequency of observing was determined by the priorities of different PTF fields and the weather. As a result, each PTF field was observed a maximum of five times each night. The log of PTF observations of Stripe 82 carried out as part of our coordinated program is shown in Figure 3. Standard exposure time per frame is $60 \mathrm{~s}$, yielding $3 \sigma$ limiting magnitudes of 20.5 and 21 in the $R$ and $g$ band respectively.

\section{RADIO DATA PROCESSING}

With the new wideband system in place for the Jansky VLA, the data rates are quite large, and this poses a computational challenge in terms of rapid data processing needed for triggered transient follow-up. The size of each of our observation blocks (listed in Table 2) is about $250 \mathrm{~GB}$, and to enable near-real-time data processing, dedicated computational hardware was set in place at the NRAO in Socorro. In general, the calibration, source cataloging, and transient search were carried out over a single cluster node at Socorro, and the imaging was distributed across multiple (up to six) cluster nodes. All this processing was done using a custom-developed, semi-automated AIPSLite/Python-based pipeline developed at Caltech (e.g., Bourke et al. 2014). Details of the near-real-time data processing are given in Section 3.1. After the completion of all observing epochs, a more detailed calibration and imaging of the raw data was carried out using ${ }^{15}$ CASA 4.1 (Section 3.2).

\subsection{Near-real-time Processing}

For each observing block, the data were downloaded onto the NRAO lustre file system (typical download time of 20 minutes) immediately after they appeared on the VLA archive (only several minutes lag after the completion of the observations). Next, the raw data were calibrated on a single cluster node using AIPS/Obit tasks accessed via ObitTalk, ParselTongue, and AIPSLite. The calibration part of the pipeline follows the procedures outlined in Chapter $\mathrm{E}$ of the AIPS Cookbook. The first three spectral windows (SPWs), spanning 2.00-2.38 GHz in frequency, and the last three SPWs, spanning 3.62-4.00 GHz, were completely flagged. Calibration as per the prescription is carried out on a per-SPW basis and almost always failed for many of these SPWs. This was caused by strong satellite-induced RFI in these bands that was not automatically removed by RFLAG. Therefore, to speed up the data processing and meet the goal of rapid follow-up, we flagged these SPWs. For the fourth epoch, we recognized that the first SPW was recoverable with minor manual flagging and the entire SPW was not flagged.

Post-calibration imaging was carried out somewhat differently for the first three epochs and the fourth epoch, since different observing modes were used. For the first three epochs, the derived calibration was applied to each pointing during imaging. The imaging stage of the pipeline was distributed over 16 cores across two cluster nodes such that, at any point in time, 16 pointings were being imaged simultaneously using the AIPS task IMAGR. CLEANing was done with 800 iterations

\footnotetext{
$\overline{15}$ http://casa.nrao.edu/. Although calibration and imaging were seen to be faster in AIPSLite than in CASA, the latter was preferred owing to the userfriendliness of plotting the data and the availability of wide-band wide-field imaging algorithms. Through our tests, no significant difference is seen in the quality of data calibrated using either software.
} 
Table 2

Summary of the Jansky VLA Observations

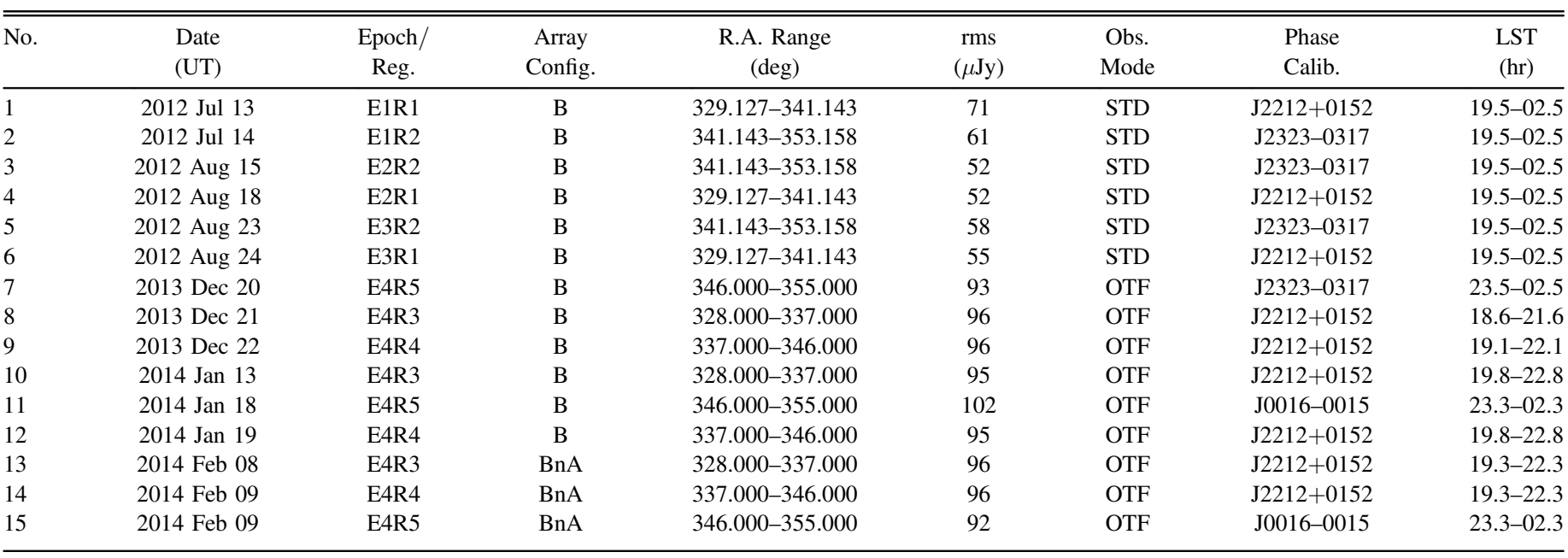

Note. The rms noise tabulated for epochs E1, E2, and E3 is before correcting the pointings for the primary beam. For the flat-sky rms noise, see Figure 6. For epoch E4, the rms noise for the individual sub-epochs from the near-real-time processing are tabulated. See Figure 6 for the rms noise of the final E4 co-add.

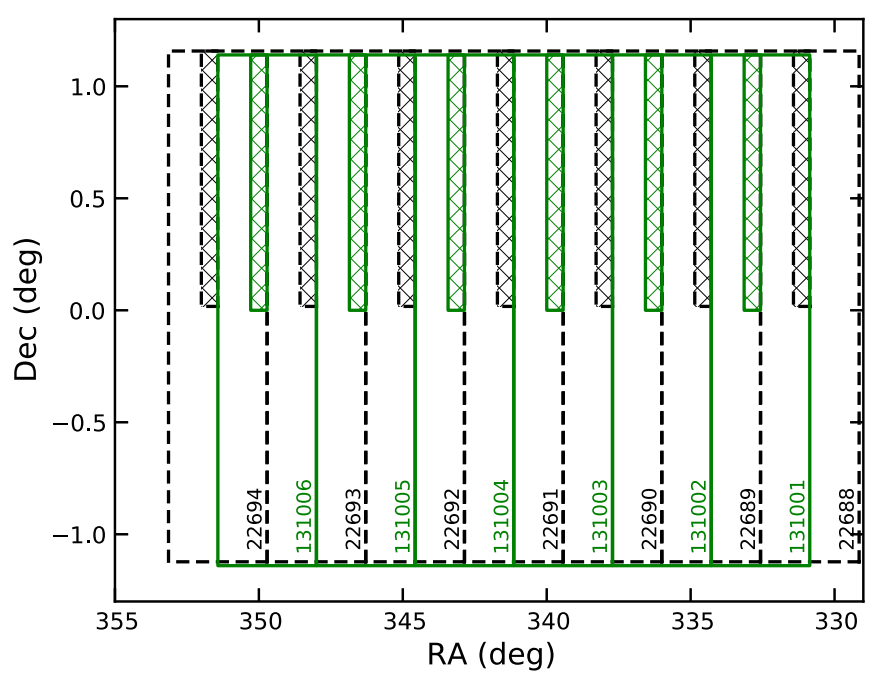

Figure 2. Setup of pointings for the PTF survey. The dashed and solid lines indicate the footprint of the different pointings. The PTF field IDs for the pointings are shown at the bottom right corner of the respective footprints. The shaded regions indicate the missing CCD.

per pointing and natural weighting. For each pointing, the CLEANed $4096 \times 4096$ pixels $^{2}, 0$ ". 58 pixel $^{-1}$ images, uncorrected for the primary beam to get a uniform rms noise across the image, were sub-imaged to $2048 \times 2048$ pixels $^{2}$ using the AIPS task SUBIM. After the imaging of each observing block, the 485 single-pointing sub-images were copied over to a local machine at Caltech, where the source cataloging and transient detection part of the pipeline was run. The thermal noise in each pointing is expected to be $\sim 50 \mu \mathrm{Jy}$, and our automated flagging produced data that are within $10 \%$ of this value, except for E1, where larger RFI is present. For source finding, the use of the sfind algorithm from MIRIAD was motivated by the short runtime and reasonable completeness and reliability of catalogs (Hancock et al. 2012; Huynh et al. 2012; Mooley et al. 2013). We cataloged all sources above the $5 \sigma$ threshold

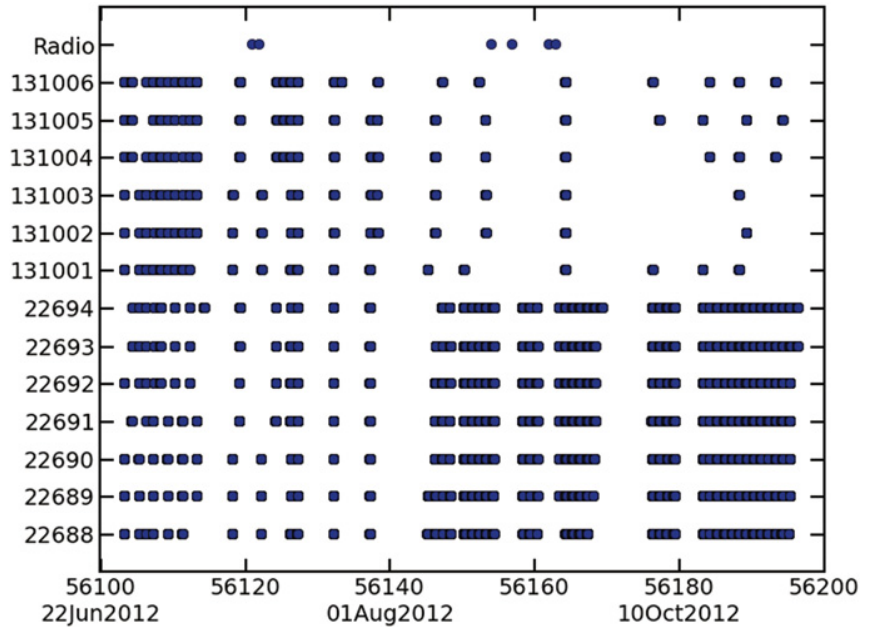

Figure 3. Log of PTF observations. MJD/Gregorian date is on the $x$-axis, and PTF field ID is on the $y$-axis. Each point corresponds to an observation. The first three Jansky VLA epochs (Table 2) are shown at the top for reference. See Section 2.2 for details.

and used these for the variability and transient search described in detail in Section 5.1.

The imaging for the fourth epoch was carried out with the CASA task clean after the calibrated data from AIPSLite were exported into UVFITS format and then imported into CASA as measurement sets. Before the imaging step, we clipped the visibility amplitudes of all SPWs at a threshold determined by the SPW with the least RFI. For each SPW, an RFI-proxy value was calculated as the sum of the mean and three times the standard deviation of the scalar-averaged amplitude. The clipping amplitude was then chosen to be the least of the RFI-proxy values. We carried out tests on several pointings to ensure that this clipping threshold was robust and did not alter the flux densities and spectral indices of sources. After the clipping of visibility amplitudes, all $4 \mathrm{~s}$ long scans, which have unique phase centers, were imaged individually. Imaging was distributed over 60 cores across five cluster nodes such that, at any given time, 60 pointings were being imaged 
simultaneously using the CASA task clean. CLEANing was done with 500 iterations per scan and natural weighting with a cyclefactor $^{16}$ of 4.5 chosen to mitigate imaging artifacts. A common $3^{\prime \prime}$ circular restoring beam was chosen to facilitate the co-addition of scans in the image plane. $2800 \times 2800$ pixels $^{2}$, 0 ."75 pixel $^{-1}$ images, uncorrected for the primary beam, were produced and then sub-imaged to $1700 \times 1700$ pixels $^{2}$ using the image analysis toolkit in CASA. A value of 1700 pixels corresponds to $20 \%$ of the primary beam at $3 \mathrm{GHz}$. The CASA images were exported to FITS format and combined into a mosaic using the AIPS task FLATN after correcting for the primary beam. The mean rms noise is $\sim 95 \mu \mathrm{Jy}$, while the theoretically expected value is $80 \mu \mathrm{Jy}$. The increased rms noise is likely due to low-level RFI and joint deconvolution not being incorporated during the CLEANing process. Sources beyond $5 \sigma$ were found using RMSD and SAD tasks in AIPS to get reasonably complete and reliable catalogs (Mooley et al. 2013). Once all the E4 observations were complete, the images were combined using the AIPS task $C O M B$ to make a final E4 mosaic. This mosaic was used to carry out a deeper variability and transient search with respect to epoch E2, the FIRST survey, and the VLA-Stripe 82 survey (Section 5).

Since computing time required for the various stages of data processing and transient search is critical for any near-real-time search, we briefly describe this aspect here. Data $\mathrm{I} / \mathrm{O}$ is the rate-determining factor in our near-real-time data processing. As a result, the processing time is a strong function of the percentage of free disk space on the NRAO lustre file system at Socorro that hosted the data processing for our survey. For the first three epochs, the lustre had $75 \%$ free disk space, and the calibration, imaging, cataloging, and transient search for each observing block, covering $25 \mathrm{deg}^{2}$ over 485 pointings or scans and amounting to $250 \mathrm{~GB}$ of visibility data, required about 4.5 , $0.5,0.5$, and $0.5 \mathrm{hr}$, respectively. Thus, we were able to detect transient candidates within $6 \mathrm{hr}$ from the completion of observations. This fast turnaround allowed same-night triggering of optical follow-up and also rapid broadband radio followup, which are crucial for understanding transients that evolve on relatively short timescales. For the fourth epoch, the lustre had 15\%-20\% free disk space and the imaging problem was much more lengthy owing to the 2025 scans to be imaged. The calibration and imaging for each observing block within E4, which had $250 \mathrm{~GB}$ of visibility data and covered a $22.5 \mathrm{deg}^{2}$ area on the sky, required 12 and $3 \mathrm{hr}$, respectively, while the transient search was completed in $1 \mathrm{hr}$.

\subsection{Final Processing}

During the near-real-time processing, the data editing and source identification (Section 3.1) were not done optimally in order to enable rapid follow-up of interesting radio transient candidates. Also, some problems with a small subset of the data were discovered after the near-real-time processing. Specifically, (1) the automated RFI algorithm and manual flagging likely excised both terrestrial interference and valid astronomical data, (2) the gain calibration for several pointings was affected by strong satellite signals, and (3) a subset of the initial calibrated data suffered from a systematic phase distortion

\footnotetext{
16 The cyclefactor parameter determines the number of minor cycles carried out between successive major clean cycles. A large cyclefactor value thus minimizes the confusion between sidelobes and true sources during minor cycles that may sometimes result in strong line or spike-shaped imaging artifacts.
}

(Section 3.2.3). Thus, following the completion of the observing epochs E1, E2, and E3, we carried out a more detailed processing of the raw data using CASA. Epoch E4 was not subjected to final detailed processing; calibrated data from the near-real-time processing were directly used for analysis.

\subsubsection{Calibration}

The final calibration was carried out using the NRAO calibration pipeline (pipeline version 1.2.0 implemented in CASA 4.1), modified to accommodate manual flagging and additional calibration diagnostic plots, and to bypass Hanning smoothing. The calibration was done with two iterative runs of the pipeline. Diagnostic plots for the flux and gain calibrators (plots of the gain calibration tables and calibrated phases and amplitudes) from the initial run were used to determine visibilities with incorrect amplitude and/or phase calibration or bad calibrator data. Antenna-, baseline-, correlation-, SPW-, and time-based manual flagging of the flux and gain calibrators was incorporated in the second run to remove these visibilities. Especially, five SPWs having frequencies between $2.12-2.38 \mathrm{GHz}$ and $3.62-4.00 \mathrm{GHz}$, for which the amplitude and phase calibration failed in most instances, were manually flagged before calibration. After the second iteration of the NRAO calibration pipeline followed by imaging, two issues persisted: (1) the amplitude gain solutions were not stable as a function of time likely owing to RFI-induced nonlinearities in the signal chain (see Section 3.2.2), and (2) a Y-shaped imaging artifact in the first 95 scans of the 2012 July 24 epoch, indicating bad raw phase data for the gain calibrator (see Section 3.2.3).

$$
\text { 3.2.2. } R F I
$$

Since Stripe 82 is close to the Clarke belt, radio observations are prone to severe RFI from satellites in geostationary and geosynchronous (GSO) orbits. The RFI in the frequency range $3.62-4.00 \mathrm{GHz}$ is low level in amplitude, but it distorts the phase information quite significantly. Those data that are irreparably affected are flagged for our final reduction. GSO satellites seen by the Jansky VLA have not been individually characterized in terms of downlink frequencies and polarizations, and our target data could potentially be affected.

The derived calibration also shows instability in the form of sporadically varying amplitude gains from the gain calibrator. This effect is pronounced in those gain calibrator scans that are severely affected by RFI. In epochs E1, E2, and E3, where the observations were carried out over the same LST range (Section 2.1), the aberration in gain is reproducible within a specific LST range. This suggests that the aberrant amplitude gains occur at a particular range of azimuth: between $124^{\circ}$ and $255^{\circ}$ for region R1 (J2212+0152 used as gain calibrator) and between $135^{\circ}$ and $220^{\circ}$ for region R2 (gain calibrator J2323-0317). The scans of the southern calibrator, J2323-0317, are more severely affected than those of J2212 +0152 owing to the proximity of the former to the Clarke belt. The left panel of Figure 4 shows the normalized gain amplitudes for J2323-0317 and the normalized rms noise in the target pointings as a function of the observing scan number (as a proxy for time). It is evident that the amplitude gains are correlated with the rms noise of the target pointings, suggestive of anomalous gain values. Our analysis of the calibrated data is complicated by the fact that, although the RFI is localized to 

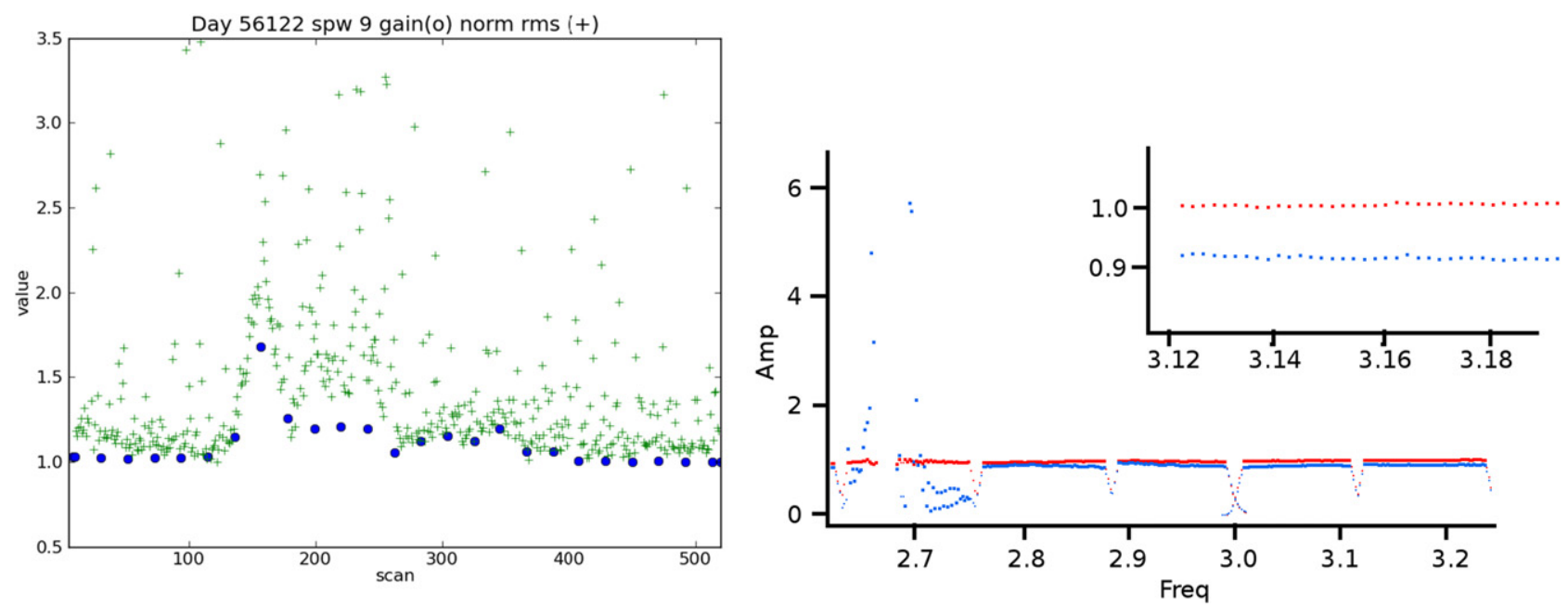

Figure 4. Demonstration of gain compression in the data. Left: the inverse of gain amplitudes derived for the gain calibrator J2323-0317 observed during the survey epoch 2012 July 14 (E1R2; blue circles) correlates well with the rms noise (green "plus" symbols) in the pointings (scans). The gain amplitudes and the rms have been arbitrarily normalized. Note that the scan number is a proxy for time. The large rms noise relative to the gain amplitudes between scans $200-250$ is likely due to large amount of low-level RFI. Right: calibrated and normalized amplitude of J2323-0317 (scan 157) observed during the 2012 July 23 (E3R2) survey epoch. RR and LL correlations are shown in red and blue, respectively. There is large RFI in the LL correlation of SPW 5 (between 2.65 and $2.75 \mathrm{GHz}$ ), which has affected the gains in the other spectral windows as well. The inset shows a zoom-in for SPW 9 (known to be RFI-free), where the gains in the LL correlation are seen to differ from the RR by almost $10 \%$ (the latter is assumed to have the true or expected gain).

typically a single SPW, amplitude gains in SPWs that are free from RFI are also significantly affected (irrespective of the baseband in which the RFI is present; right panel of Figure 4). We refer to this as "gain compression." The "compression" signifies that the amplitude gain values are reduced with respect to their true values and result in increased rms noise in the target fields. Initial assessment attributes the aberrant gains to nonlinearities in the amplification stage in the analog signal chain somewhere upstream from the correlator caused by the the high power of the satellite signals. However, this hypothesis has not been thoroughly tested.

We devised a fix for the gain compression in the gain calibrator scans by altering the amplitude gain calibration table generated by the NRAO calibration pipeline. Toward the end of each epoch there is a time interval lasting several minutes where the gains are relatively stable and close to unity (to within a few percent). We therefore applied constant amplitude gains of unity for the duration of each epoch, and for all epochs. With this correction, the rms noise values for epochs $\mathrm{E} 1, \mathrm{E} 2$, and $\mathrm{E} 3$ are more stable with time and are usually within $10 \%$ of the thermal noise. The spread in the baseline-based amplitude gains in the stable time duration is $<0.05$, and hence we estimate that this correction will affect the true amplitudes of the target sources by $<5 \%$. Given that gain calibrators in the vicinity of the Clarke belt are susceptible to gain compression, we have avoided using such gain calibrators for the final CNSS survey. Accordingly, there is no indication of severe gain compression in the gain calibrator scans from epoch E4. While the gain compression in the gain calibrator scans has a relatively straightforward workaround, the effect of gain compression on the target pointings, if present, is much more challenging to deduce. We investigate the possible issue of gain compression in target fields in Section 5.

\subsubsection{Imaging}

After calibration of the data using the NRAO pipeline, we clipped the visibility amplitudes of all SPWs in the manner described in Section 3.1. We then derived and applied a singleround phase-only self-calibration solution to every pointing containing a source brighter than $10 \mathrm{mJy}$ in the near-real-time image. The model for the self-calibration was constructed by making a preliminary image with 100 clean iterations using the CASA task clean. Self-calibration solutions were allowed to break at SPW and scan boundaries. Each self-calibrated pointing was then imaged by cleaning within the $1 \%$ power point of the primary beam of the lowest frequency, $2.0 \mathrm{GHz}$, using natural weighting in the clean task in CASA, choosing a cell size of 0 ". 6 and 500 clean iterations to minimize clean bias. A single Taylor term (nterms $=1$ ) was used, which was sufficient to achieve the desired dynamic range $\sim 10^{3}$. We also chose a cyclefactor of 4.5 to minimize imaging arifacts. Basic quality checks were made by visually inspecting the images for each pointing. Using Briggs weighting with a robust parameter of zero, we re-imaged those pointings in which imaging artifacts persisted. All the $4800 \times 4800$ pixels $^{2}$ cleaned singlepointing images at $2.8 \mathrm{GHz}$ were then sub-imaged to $2400 \times 2400$ pixels $^{2}$. A value of 2400 pixels corresponds to $42 \%, 15 \%$, and $1 \%$ of the power point of the primary beam at 2.0, 2.8, and $3.63 \mathrm{GHz}$, respectively (Figure 5). The median synthesized beam obtained for all three epochs is 3 !" $1 \times 2$ ". 0 , except for E1R1, where the median synthesized beam size is 3 ". $8 \times 2$ "! 1 likely owing to increased RFI. The mean rms noise per epoch is given in Table 2. The cumulative plot for rms noise (flat sky) as a function of survey area is shown in Figure 6. For epochs E2 and E3, more than $90 \%$ of the survey area has rms noise lower than $105 \mu \mathrm{Jy}$, while for $\mathrm{E} 4$ it is better than $75 \mu \mathrm{Jy}$. Epoch E1, having rms noise of $130 \mu \mathrm{Jy}$ or better over $90 \%$ of the survey area, is severely affected by low-level RFI compared to other epochs. Note that, for the first three epochs, neighboring pointings are quite far apart and do not contribute significantly to the sensitivity of the overlap region. Hence, single pointings were imaged separately and treated independently during the source finding step.

For the 2012 August 24 (epoch E3, region R1) epoch, the first $1.5 \mathrm{hr}$ of observations have been affected by a systematic 


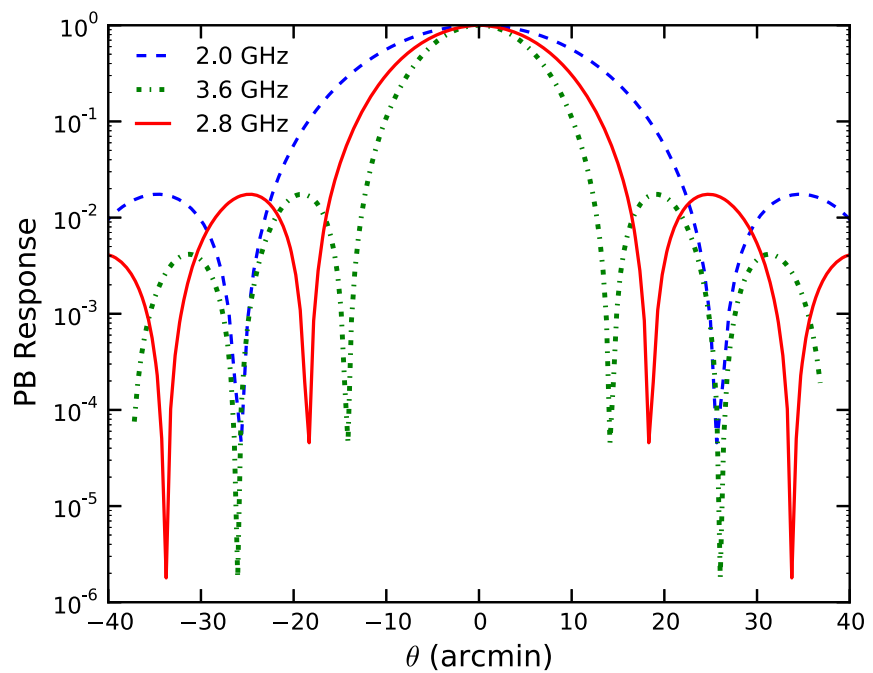

Figure 5. The primary beam response at the lowest (blue dashed line), highest (green dot-dashed), and reference (solid red) frequencies.

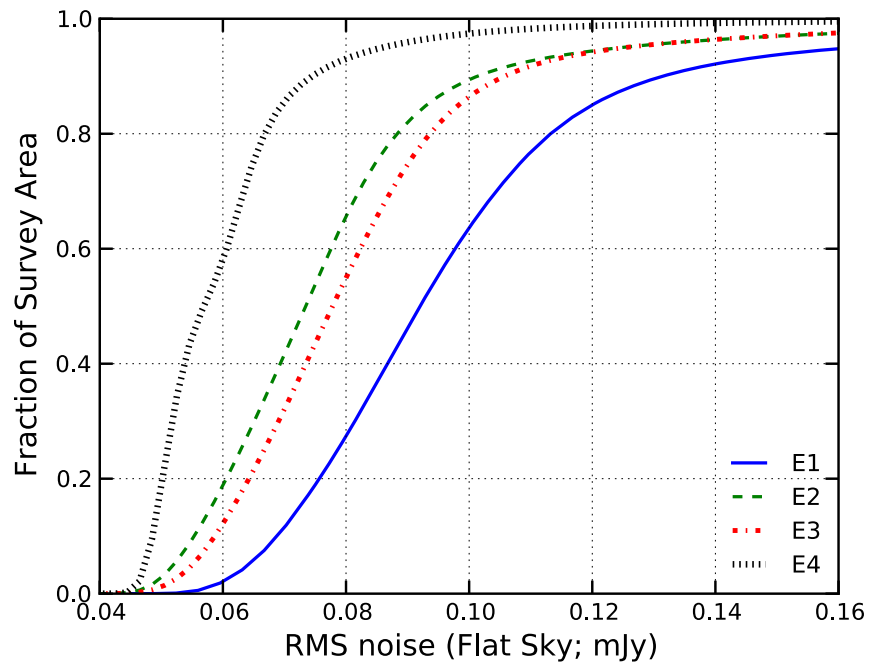

Figure 6. The survey area having the rms noise (flat sky) less than or equal to the given sensitivity.

phase distortion. About 80 target pointings and six phase calibrator (J2212+0152) scans have been thus affected. When the calibrated data are imaged, the phase distortion manifests as a three-pronged artifact such that point sources appear extended along three axes characteristic of the $\mathrm{Y}$ shape of the VLA configuration (left panel of Figure 7). We looked at the phase gain solutions per antenna derived from one of the affected phase calibrator scans and plotted it as a function of $x$ - and $y$ positions (east-west and north-south) of the respective antennas in the array. The center of the array has higher gain phase (degrees) than the arms, and along the arms, it decreases monotonically, in general. This is equivalent to a bowl-shaped or lenticular phase screen above the array. The exact cause of the phase distortion is unknown, but it may arise as a result of RFI, unfavorable weather, the ionosphere, or some internal error in the antenna phase recording. While the true phases can be restored through self-calibration, not all our affected pointings have bright sources to facilitate this. Therefore, for the affected pointings, we used the corresponding phases and amplitudes from the 2012 July 18 (epoch E2, region R1) observations as a model for self-calibration. The image of a bright source after such a calibration is applied is shown in the right panel of Figure 7 .

\subsubsection{Source Catalogs}

The AIPS task RMSD was used to create an rms image for each individual pointing for epochs E1, E2, and E3, and the resulting background images were supplied as input to SAD. Source finding was then carried out with SAD to find sources with signal-to-noise ratio $(\mathrm{S} / \mathrm{N})>5$. For sources present in adjacent pointings, only the catalog entries were retained in which the sources are closest to their respective pointing centers. Thus, the cataloged sources are within $9^{\prime}$ (within $40 \%$ of the $2.8 \mathrm{GHz}$ primary beam) from their nearest pointing centers. Approximately $10^{9}$ synthesized beams span the images from our four-epoch survey, from which we expect 500, 2, and $<1$ noise peaks above $5 \sigma, 6 \sigma$, and $7 \sigma$, respectively. While we used $5 \sigma$ source catalogs for the near-real-time transient search, we followed the recommendations of Frail et al. (2012), to filter our new source catalogs and keep only $\mathrm{S} / \mathrm{N}>7$ sources during the final processing. With the goal of variable and transient search in mind, we further retained only probable point sources. ${ }^{17}$ The resulting catalogs from each epoch were then merged into a single point-source catalog (PSC).

In many cases, it was found that the peak flux densities of sources in the PSC, as reported in the original SAD catalogs, were not in agreement with the peak flux densities as seen in the images. Since accurate peak flux densities are critical for any variability and transient search, we replaced the SAD peak flux density values for all objects in the PSC with the corresponding peak pixel values in the single-epoch images. The uncertainties in the peak flux densities of sources in the PSC were taken directly from their corresponding counterparts in the SAD catalogs or by measuring the image rms noise in the vicinity of the sources. The flux densities of all sources in the PSC and their associated uncertainties were corrected for the primary beam at $2.8 \mathrm{GHz}$ using parameters from the PBCOR task in AIPS. The PSC thus contains peak flux densities and the uncertainties of 3652 point sources having $S / N>7$ in any one of the four epochs. The histogram in the left panel of Figure 8 shows the distribution of flux densities from epoch E2 of sources in the PSC. We used this PSC for our variability and transient search (Section 5).

\subsubsection{Data Release}

As part of a preliminary data release, ${ }^{18}$ we provide to the astronomical community single-epoch images and our PSC for the four epochs of radio observations of the survey region.

\footnotetext{
${ }^{17}$ Sources having the ratio of their integrated flux densities to peak flux densities less than 1.5 were selected as probable point sources. During this step of filtering the source catalogs, we also rejected probable false positives associated with imaging artifacts in the vicinity of bright sources. Sources in the catalog that were elongated by a factor of 2.5 larger than the ratio of the major axis to minor axis of the convolved beam were rejected. All cataloged objects within 1 !" 6 of $>10 \mathrm{mJy}$ sources were rejected, retaining the $>10 \mathrm{mJy}$ sources themselves. In order to filter out false positives with flux densities greater than $10 \mathrm{mJy}$, all cataloged objects within 1 "' 6 of $>50$ mJy sources were rejected, retaining the $>50 \mathrm{mJy}$ sources themselves. This reduces our transient search area by less than one square degree, but gets rid of almost most false positives. Some sidelobes identified as such by eye were also rejected from the source catalogs. This false-positive rejection step eliminates 438 objects out of 4205 unique objects in our list of probable point sources. An additional 35 sources were discarded as being resolved after manual inspection of a subset of images from our survey.

${ }^{18}$ http://tauceti.caltech.edu/stripe82
} 

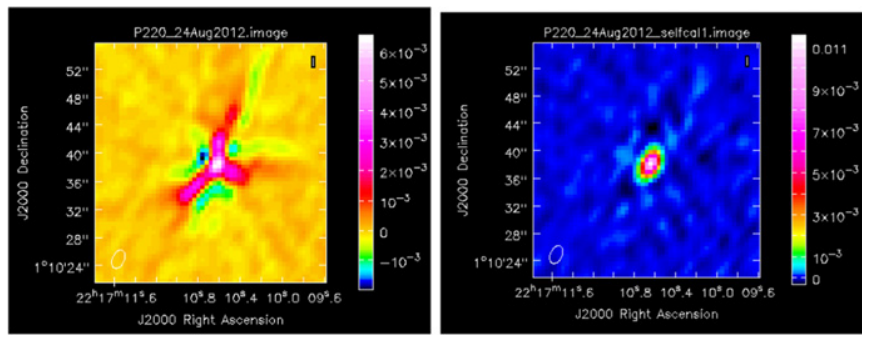

Figure 7. The Y-shaped imaging artifact discovered in the first $1.5 \mathrm{hr}$ of observations for the 2012 August 24 (E3R1) epoch. The image of a bright point source before (left) and after (right) the calibration described in Section 3.2.3. The field of view in both panels is identical and equal to $35^{\prime \prime}$. In the left panel, the color bar runs from -2.0 to $+6.5 \mathrm{mJy}$, while in the right panel it runs from -0.5 to $+12.0 \mathrm{mJy}$.
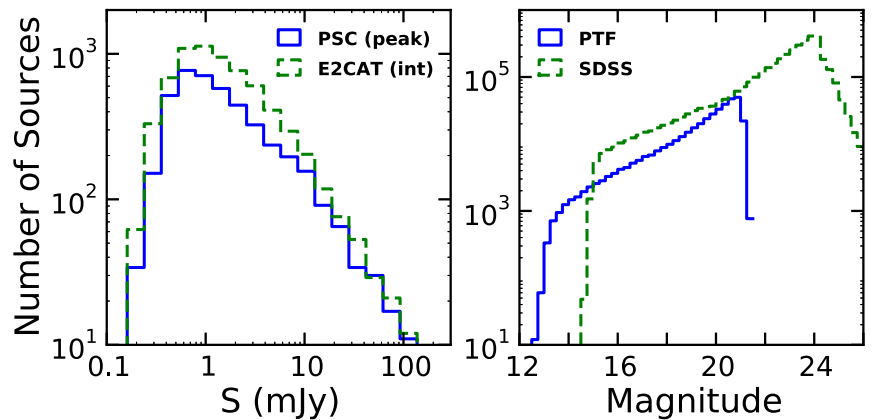

Figure 8. Left: histograms of the peak flux densities in epoch E2 of sources in the PSC and integrated flux densities for all sources in E2CAT. Right: PTF $R$ band and the SDSS (Stripe 82 co-add) $r$-band magnitudes of the optical sources considered in this work. The SDSS histogram between magnitudes 15 and 21 is higher than the PTF histogram owing to multiple entries of sources in SDSS in this magnitude range. Below a magnitude of 16, the inconsistency is due to the different saturation levels of the SDSS and PTF CCDs.

Additionally, we release a $5 \sigma$ catalog (E2CAT) of sources from epoch E2. E2CAT consists of 6846 sources after a preliminary elimination of false positives (probable sidelobes) as done with the PSC and described in Section 3.2.4. The histogram of the integrated flux densities recorded in E2CAT is shown in the left panel of Figure 8. The final data release, consisting of calibrated UV data, a deep mosaic made from the co-addition of all four epochs, and a deep source catalog, will be done in a later publication (A. Horesh et al. 2016, in preparation).

\section{OPTICAL DATA PROCESSING}

The PTF near-real-time reduction and transient search were carried out using a pipeline hosted at the Lawrence Livermore Berkeley Laboratory. Transient search was done by subtracting an older reference (deep co-add) image from the new observations. For further details on the PTF near-real-time operations, see Section 2.1 of Smith et al. (2011). The Stripe 82 data processed with the IPAC pipeline (Laher et al. 2014) and photometrically calibrated (Ofek et al. 2012) were used to make light curves reported in this work. The processed data and catalogs are available online at the NASA/IPAC Infrared Science Archive ${ }^{19}$ as part of PTF first data release. The histogram of the $R$-band magnitudes of PTF sources considered

\footnotetext{
$\overline{19}$ http://irsa.ipac.caltech.edu/applications/ptf
}

in this work are shown in the right panel of Figure 8 . The distribution of $r$-band magnitudes from the SDSS Stripe 82 coadd catalog is also given in Figure 8.

\section{RADIO VARIABLES AND TRANSIENTS}

In this paper we use the terms "variables" and "transients" somewhat interchangeably, making note of the fact that the processes listed in Table 1 are all transient processes. Here we use our four survey epochs to carry out two-epoch transient searches on different timescales. We are thus probing variability on all timescales that are approximately larger than the duration of each observation ( $40 \mathrm{~s}$ per pointing in our case) but less than the separation between the two epochs being compared. Accordingly, in the text below, we refer to a transient search on a "timescale of X days" synonymously with "timescale less than X days," but note that in Table 1 we refer to the precise "timescale of evolution" since the evolution of the light curves of these transients has been well studied through high-cadence targeted observations.

One of the primary goals of our survey was to understand the transient phenomena on timescales of 1 week, 1 month, and 1 yr. For this purpose, we performed a two-epoch comparison of flux densities of sources in our survey using the PSC from Section 3.2.4. Since radio interferometric noise is Gaussian distributed (e.g., Condon et al. 1998), we can compare the flux densities of a source between two epochs with the statistic $\left(S_{1}-S_{2}\right) / \sqrt{\sigma_{1}^{2}+\sigma_{2}^{2}}=\Delta S / \sigma$ under the null hypothesis that they are drawn from the same distribution (and hence the source is nonvariable between the two epochs that are being compared). From statistical theory we know that this quantity is distributed according to the Student's- $t$ distribution (Student 1908; Bevington \& Robinson 2003). We define a source as being a variable if the $t$-statistic lies beyond the $95 \%$ confidence interval, ${ }^{20}$ i.e., if

$$
V_{s}=\left|\frac{\Delta S}{\sigma}\right| \geqslant 4.3 .
$$

The choice of the confidence interval was motivated by the need for high reliability of the variable sources found, while making a possible compromise on the completeness. This issue of completeness and reliability is taken into account while calculating the rates of variable sources in Section 7.1. For the two-epoch comparison of variables selected using Equation (1), we use the modulation index as the measure of variability:

$$
m=\frac{\Delta S}{\bar{S}},
$$

where $\bar{S}$ is the mean of the flux densities, $S_{1} \pm \sigma_{1}$ and $S_{2} \pm \sigma_{2}$, in the two epochs being compared. We note that the definition of modulation index used here is proportional to the one used by Mooley et al. (2013), but larger by a factor of $\sqrt{2}$. The modulation index is related to the fractional variability, $f_{\mathrm{var}}$, by

\footnotetext{
${ }^{20}$ For two degrees of freedom this corresponds to a Gaussian probability of more than $\pm 4 \sigma$. For the Gaussian distribution, $4 \sigma$ corresponds to a probability of about $1 / 16,000$, while the number of measurements in our variability analysis (few thousand point sources multiplied by four epochs) is about 15,000 . For our variability analysis we could have used the $\chi^{2}$ statistic as previous studies have done (e.g., Ofek et al. 2011; Mooley et al. 2013), but for cases where the number of degrees of freedom are small, Student's $t$ is preferred. See also chapter 4 and Appendix C of Bevington \& Robinson (2003).
} 


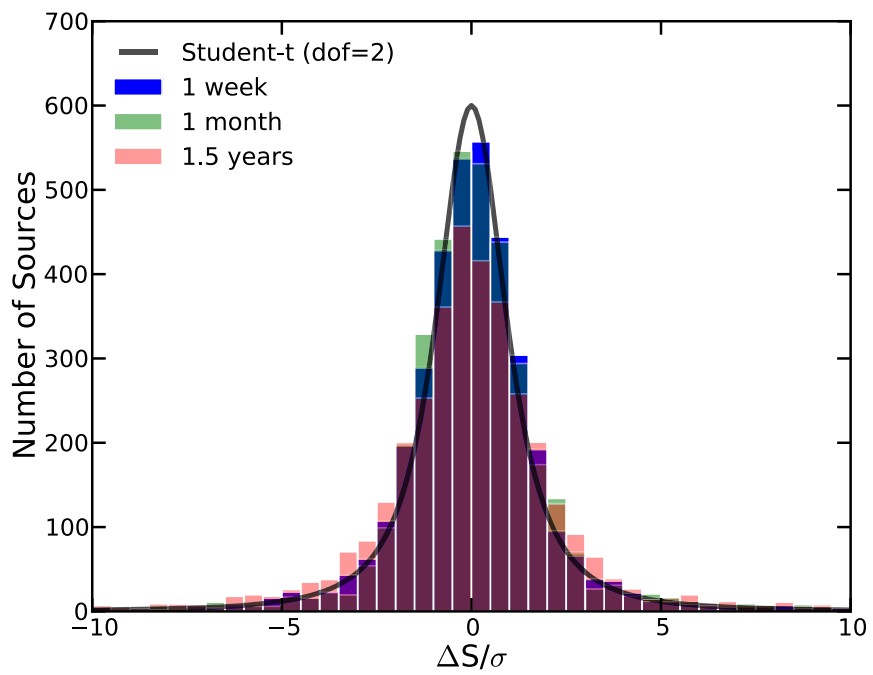

Figure 9. A histogram of the variability statistic, $V_{s}$, for sources in the PSC After the application of the corrections to the flux densities mentioned in Section $5, V_{s}$ agrees with the expected distribution, Student's $t$.

the following equation:

$$
m=2 \times \frac{S_{1} / S_{2}-1}{S_{1} / S_{2}+1}=2 \times \frac{f_{\mathrm{var}}-1}{f_{\mathrm{var}}+1} .
$$

\subsection{Near-real-time Search}

Initially, during the near-real-time transient and variability search, we used variability statistic $V_{s}$ (Equation (1)) and further selected sources with fractional variability larger than $50 \%$. As soon as a new epoch was observed, it was compared with all previous epochs. Among 6000 sources in our nearreal-time $5 \sigma$ single-epoch catalogs, we found $\sim 500$ to be variables. We note here, beforehand, that following the final data processing, we found that most of these candidates were falsely identified as variable owing to the RFI-induced amplitude calibration problem identified in Section 3.2.2.

The shortlist of follow-up candidates was generated from the near-real-time catalogs. For further filtering of variable candidates, we used multiwavelength archival data and PTF and inspected the radio image cutouts by eye. It turned out that the PTF data were not very useful for this purpose. As we show in Section 6, there is little overlap between variable radio sources and sources that vary in the optical, and almost all these jointly variable sources are AGNs. Optical spectra from the SDSS BOSS program (Dawson et al. 2013) were especially useful in filtering our list of candidates. In general, for those radio transients having BOSS spectra, we filtered out sources showing AGN features in their optical spectra. WISE colors were also used to eliminate possible AGNs. Preference was given to sources that are undetected in archival radio data.

We also compared our catalogs with those from past surveys at $1.4 \mathrm{GHz}$, viz., FIRST (White et al. 1997) and VLA-Stripe 82 (Hodge et al. 2011), and selected $\sim 10$ point sources with implied spectral indices more than 2.5 or less than -2.5 between 3 and $1.4 \mathrm{GHz}$. This was motivated by the search for transients such as supernovae and tidal disruption events (Swift J1644+57-like; Zauderer et al. 2011, 2013) that evolve on a timescale of years. However, most of these candidates turned out to be either GPS sources or flaring AGN's (see below).
Our final list of candidates for follow-up consisted of 20 objects that displayed a factor of two or more fractional variability between any two epochs, or were new bright sources compared with previous surveys at $1.4 \mathrm{GHz}$.

\subsection{Search for Variables and Transients after Final Data Processing}

After correcting for the aberrant complex gains during the final data processing (Section 3.2), we carried out a careful search for variables and transients bearing in mind the possibility of gain compression (Section 3.2.2) in the target fields. To maintain consistency during our variability and transient search, we only compared epochs E1, E3, and E4 with epoch E2 in order to probe variability on timescales of 1 month, 1 week, and $1.5 \mathrm{yr}$, respectively. Note that among the first three epochs, E2 was the least affected by RFI.

For each source in the PSC, we calculated the statistical quantity $V_{s}$ using Equation (1). There appeared to be a small deviation of this statistic from the expected distribution (Student's $t$ distribution). This may be a result of low-level gain compression in the target fields. Although the epoch-toepoch flux densities are not significantly discrepant, we addressed this issue by applying small, relative corrections to the ratios of and the differences in the flux densities of sources between the two epochs being compared.

For the 1 week and 1 month comparison, we made multiplicative corrections of $2 \% \pm 3 \%$ (median value with standard deviation) to the ratios of flux densities and additive corrections of $20 \mu \mathrm{Jy} \pm 50 \mu \mathrm{Jy}$ to the differences in the flux densities of sources in the PSC. These corrections were derived for the two-epoch comparisons by plotting the ratios and differences of flux densities of sources as a function of time of observation and demanding the ratios and differences to be centered at unity and zero, respectively. During the comparison between epochs E4 and E2, we found it necessary to make two independent sets of corrections to the ratios of flux densities. In the first set, multiplicative corrections of $3 \% \pm 4 \%$ were seen to be correlated with the decl. of the sources. This discrepancy of flux densities in epoch E4 with respect to epoch E2 is a result of the the OTF survey design and the near-real-time imaging process and will be described in detail in CNSS Paper II. In short, during the OTF observations carried out for epoch E4, the primary beam moved by $4^{\prime}$ within every scan; this was not accounted for in the imaging step. The resulting fractional change in flux density of a source depends on its position in the primary beam. This effect is manifested as a sinusoidal pattern when the flux density ratios are plotted as a function of source declinations. The second set of corrections consist of multiplicative corrections of $4 \% \pm 4 \%$ to the ratios of flux densities and may be attributed to gain compression. Additive corrections of $10 \mu \mathrm{Jy} \pm 30 \mu \mathrm{Jy}$ were applied to the differences in the flux densities between epochs E4 and E2. With the application of these small, first-order corrections we ensure to not overfit the data and remove real variability in flux densities of the sources in the PSC. The histogram showing the resultant distributions of the variability statistic, $V_{s}$, is shown in Figure 9. It is evident that, after the corrections, $V_{s}$ agrees well with the Student's t distribution (however, see note on the $1.5 \mathrm{yr}$ distribution below).

To determine additional sources of artificial variability, we ran further intensive tests with manual flagging and imaging. We found variations of up to a few percent in the flux densities 


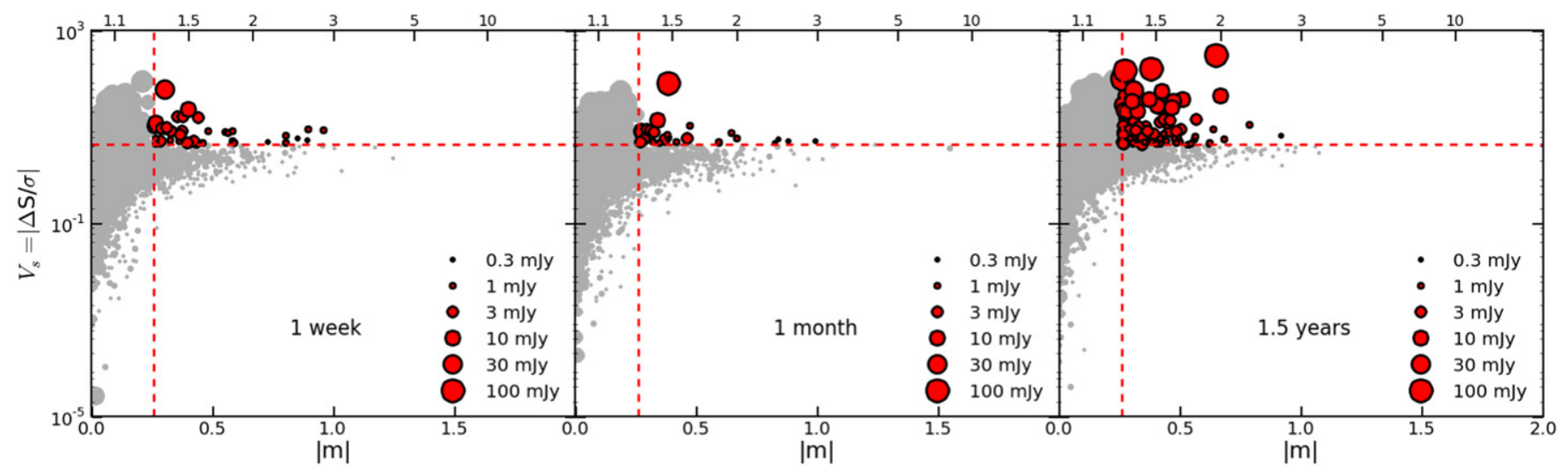

Figure 10. The variability statistic, $V_{s}$, as a function of the modulation index, $m$, for sources in the PSC for the various timescales considered in this work. The dashed red lines indicate our selection criteria for variables. Filled gray circles denote sources that are not variable, while red circles have been selected as variables. The sizes of the circles indicate the mean flux densities of the sources in the two epochs being compared. We find 38, 31, and 96 variable sources on timescales of 1 week, 1 month, and $1.5 \mathrm{yr}$, respectively, amounting to a total of 142 variable sources. The upper $x$-axis in each of the three panels represents the fractional variability as given by Equation (3). See Section 5 for details.

of sources just by choosing different values for the input parameters to the CASA clean task. Specifically, these parameters are cleaning iterations (niter) and Taylor terms (nterms). We also noticed variations by changing the values provided to the clipminmax parameter in the flagdata task. Additionally, we know that the flux density absolute calibration (we used 3C 48 as the flux density standard calibrator in our survey) is usually accurate to only $3 \%-5 \%$ (e.g., Ofek et al. 2011; Thyagarajan et al. 2011; Mooley et al. 2013).

All in all, owing to gain compression and other possible causes of artificial variability mentioned above, we adopt a conservative criterion for selection of our variable sources: fractional variability greater than $0.3(|m|>0.26)$. Our final variability selection criteria are $V_{s} \geqslant 4.3$ and $|m|>0.26$. The plots of the $V_{s}$ versus $m$ for sources in the PSC after applying the corrections mentioned earlier in this subsection are shown in Figure 10.

For the sources in the PSC satisfying the variability selection criteria, we manually inspected the image cutouts and their fitted Gaussian parameters, viz., major axes $(b)$, minor axes $(a)$, the peak flux densities $\left(S_{\text {peak }}\right)$, and the integrated flux densities $\left(S_{\text {int }}\right)$, in order to ensure that they are not resolved. For sources detected at a high significance level $(\mathrm{S} / \mathrm{N} \gtrsim 15)$, we used the criteria $S_{\text {int }} / S_{\text {peak }} \lesssim 1.1, \quad b \lesssim 1.1 \times \mathrm{BMAJ}$ and $a \lesssim 1.1 \times$ BMIN, where the variables in typewriter text denote the major and minor axes of the synthesized beam. For the sources detected at low significance, we relaxed the integratedto-peak flux density ratio to $1.3-1.5$. This procedure for selecting unresolved is motivated by the standard techniques used for deep radio surveys (e.g., Huynh et al. 2005; Franzen et al. 2015). While this approach (see Hodge et al. 2013) errs on the conservative side, we prefer to identify real variables rather than false positives caused by resolution effects.

We thus find 142 variables among 3652 sources in our PSC, or, in other words, about $3.8 \%$ of the sample has fractional variability more than $30 \%$ on timescales $<1.5 \mathrm{yr}$. The number of point sources varying on week, month, and 1.5 year timescales are 38, 31, and 96 respectively, or $1.0 \%, 0.8 \%$, and $2.6 \%$, respectively. These sources are listed in Table 3. A glance at Figure 9 suggests that the variability statistic, $V_{s}$, comparing epochs E2 and E4 (probing variability on a timescale of $1.5 \mathrm{yr}$ ) deviates from the Student's t distribution in the tail. This may be due to some calibration errors from the near-real-time processing that we have not tried correcting for, and therefore the fraction of variables on a $1.5 \mathrm{yr}$ timescale, $2.6 \%$, is likely an upper limit. Note that the variable sources are distributed uniformly on the sky with no pattern indicative of a particular region(s) of the sky being affected by calibration errors.

Since our $50 \mathrm{deg}^{2}$ survey region contains rich archival data from the FIRST and VLA-Stripe 82 surveys, we extended our transient search to timescales of up to $20 \mathrm{yr}$. We compared the $3 \mathrm{GHz}$ peak flux densities of sources in the PSC with the 1.4 GHz peak flux densities from these two surveys (Figure 11). The mean spectral index ${ }^{21}, \alpha_{\text {mean }}$, appears to be between -0.5 and -1 , as expected (e.g., Randall et al. 2012). With the view of finding only the extreme variable and transient candidates, we searched beyond implied spectral indices of $-1 \pm 2.5$ (i.e., less than -3.5 or greater than 1.5) with respect to the FIRST survey and beyond $-0.5 \pm 2.5$ with respect to the VLA-Stripe 82 survey. The threshold of \pm 2.5 in spectral index is somewhat arbitrary; it is motivated by our need for filtering out most of the sources and selecting only a few, extreme objects. Our search resulted in 11 unique objects. Two candidates are resolved out (closely separated radio lobes) in our data; one candidate is identified as such owing to its being erroneously absent in VLA-Stripe 82 catalog (but present in the image), and one candidate, VTC220456-000147, is identified as a variable also on a timescale of 1 month. Accordingly, out of the eight genuine variables, ${ }^{22}$ seven have been listed in Table 3 in the timescale $<20 \mathrm{yr}$ section.

Below, we discuss five candidates ${ }^{23}$ in detail that are meant to be broadly representative of our complete sample of 142 variables and transients. Additionally, we discuss about two transients found serendipitously. A summary of all the variable and transient sources found in this work is given in Table 3,

\footnotetext{
${ }^{21}$ We define the spectral index, $\alpha$, as $S \propto \nu^{\alpha}$.

22 Note that any extragalactic source variable on a timescale even as large as a decade will be a point source for an angular resolution of $1^{\prime \prime}$, given the lighttravel distance. Here we have presented only isolated point sources in our final list of variables.

${ }^{23}$ We adopt the following naming convention for our variable and transient sources: VTChhmmss+ddmmss, where "VTC" stands for "VLA transient candidate" and the R.A. and decl. are both rounded at the least significant digit.
} 
Table 3

Summary of the Radio Transient and Variable Sources Found in This Work

\begin{tabular}{|c|c|c|c|c|c|c|c|c|c|c|c|c|c|c|}
\hline VTC & $\begin{array}{l}\alpha_{\text {J2000 }} \\
\text { (deg) }\end{array}$ & $\begin{array}{l}\delta_{\text {I2000 }} \\
\text { (deg) }\end{array}$ & $\begin{array}{c}S_{\mathrm{E} 1} \\
(\mathrm{mJy})\end{array}$ & $\begin{array}{c}S_{\mathrm{E} 2} \\
(\mathrm{mJy})\end{array}$ & $\begin{array}{c}S_{\mathrm{E} 3} \\
(\mathrm{mJy})\end{array}$ & $\begin{array}{c}S_{\mathrm{E} 4} \\
(\mathrm{mJy})\end{array}$ & $m$ & $\begin{array}{l}\text { FIRST } \\
(\mathrm{mJy})\end{array}$ & $\begin{array}{l}\text { Hodge et al. (2011) } \\
(\mathrm{mJy})\end{array}$ & $\begin{array}{l}r_{\text {SDSS }} \\
(\mathrm{mag})\end{array}$ & PTF & $z_{\text {spec }}$ & $\begin{array}{c}\log \bar{L}_{\nu} \\
\left(\operatorname{erg} \mathrm{cm}^{-2} \mathrm{~s}^{-1} \text { ) }\right.\end{array}$ & Identificatior \\
\hline \multicolumn{15}{|c|}{ Timescale $<1$ week } \\
\hline 220210-000203 & 330.539900 & -0.034252 & $2.079 \pm 0.109$ & $1.064 \pm 0.077$ & $1.862 \pm 0.083$ & $1.382 \pm 0.059$ & -0.56 & $<0.50$ & & & $\ldots$ & & & AGN \\
\hline 220609-004654 & 331.536395 & -0.781711 & $3.970 \pm 0.154$ & $3.424 \pm 0.106$ & $4.637 \pm 0.112$ & $3.390 \pm 0.060$ & -0.33 & $1.83 \pm 0.14$ & $1.47 \pm 0.07$ & 20.7 & nv & 0.37 & 31.1 & AGN \\
\hline $221122+005202$ & 332.842184 & 0.867327 & $1.665 \pm 0.096$ & $2.037 \pm 0.070$ & $1.122 \pm 0.085$ & $2.325 \pm 0.041$ & 0.55 & $1.25 \pm 0.11$ & $0.52 \pm 0.07$ & 20.0 & $\mathrm{nv}$ & 0.31 & 30.7 & AGN \\
\hline $221136+011142$ & 332.898974 & 1.194886 & $3.420 \pm 0.077$ & $3.128 \pm 0.054$ & $4.383 \pm 0.064$ & $2.368 \pm 0.061$ & -0.36 & $1.64 \pm 0.14$ & $1.26 \pm 0.16$ & 24.1 & $\ldots$ & $\ldots$ & $\ldots$ & AGN \\
\hline $221226+004322$ & 333.107130 & 0.722873 & $2.408 \pm 0.067$ & $2.951 \pm 0.057$ & $2.112 \pm 0.058$ & $2.391 \pm 0.044$ & 0.31 & $2.50 \pm 0.11$ & $1.83 \pm 0.06$ & 23.1 & $\ldots$ & & & AGN \\
\hline $221406+002305$ & 333.524900 & 0.384618 & $0.809 \pm 0.063$ & $0.877 \pm 0.055$ & $0.464 \pm 0.049$ & $0.604 \pm 0.050$ & 0.58 & $<0.51$ & $<0.32$ & 18.4 & $\mathrm{nv}$ & 0.15 & 29.5 & AGN \\
\hline $221541+010856$ & 333.921049 & 1.148981 & $1.425 \pm 0.081$ & $1.041 \pm 0.053$ & $1.664 \pm 0.063$ & $1.213 \pm 0.059$ & -0.48 & $<0.69$ & $0.68 \pm 0.09$ & 19.9 & $\ldots$ & $0.32^{\mathrm{a}}$ & 30.5 & AGN \\
\hline 221713-002216 & 334.306166 & -0.371232 & $1.376 \pm 0.088$ & $1.320 \pm 0.068$ & $1.783 \pm 0.071$ & $1.027 \pm 0.053$ & -0.33 & $<0.53$ & $1.14 \pm 0.07$ & 23.8 & $\ldots$ & & & AGN \\
\hline $221913+011147$ & 334.805979 & 1.196250 & $1.015 \pm 0.085$ & $1.442 \pm 0.063$ & $0.539 \pm 0.074$ & $1.007 \pm 0.047$ & 0.90 & $1.05 \pm 0.14$ & $0.82 \pm 0.07$ & 19.1 & $\ldots$ & $0.20^{\mathrm{a}}$ & 30.0 & AGN \\
\hline 222009-010814 & 335.036567 & -1.137346 & $4.386 \pm 0.096$ & $3.873 \pm 0.071$ & $5.565 \pm 0.081$ & $4.895 \pm 0.061$ & -0.38 & $5.23 \pm 0.15$ & $3.13 \pm 0.10$ & 20.6 & $\mathrm{nv}$ & $\ldots$ & & QSO \\
\hline 222150-000325 & 335.460301 & -0.056997 & $2.239 \pm 0.121$ & $2.470 \pm 0.080$ & $1.819 \pm 0.086$ & $2.106 \pm 0.053$ & 0.29 & $2.70 \pm 0.11$ & $2.24 \pm 0.07$ & & $\ldots$ & & & AGN \\
\hline $222232+011104$ & 335.634687 & 1.184480 & $0.860 \pm 0.062$ & $0.844 \pm 0.048$ & $0.348 \pm 0.048$ & $0.944 \pm 0.048$ & 0.80 & $1.34 \pm 0.14$ & $1.50 \pm 0.06$ & 19.5 & $\ldots$ & 0.29 & 30.2 & AGN \\
\hline $222236+010556$ & 335.648844 & 1.098840 & $1.009 \pm 0.078$ & $1.054 \pm 0.056$ & $0.358 \pm 0.055$ & $0.927 \pm 0.050$ & 0.96 & $<0.72$ & $0.53 \pm 0.05$ & 18.4 & $\mathrm{nv}$ & 0.20 & 29.9 & AGN \\
\hline $222330+010227$ & 335.873148 & 1.040835 & $9.414 \pm 0.134$ & $8.921 \pm 0.091$ & $5.740 \pm 0.097$ & $5.391 \pm 0.044$ & 0.40 & $6.11 \pm 0.13$ & $6.82 \pm 0.06$ & 19.3 & $\mathrm{nv}$ & & & QSO \\
\hline $222520+004939$ & 336.331883 & 0.827551 & $3.807 \pm 0.115$ & $3.500 \pm 0.090$ & $5.326 \pm 0.085$ & $4.273 \pm 0.054$ & -0.44 & $4.69 \pm 0.12$ & $3.76 \pm 0.08$ & 22.3 & $\ldots$ & $0.80^{\mathrm{a}}$ & 31.9 & AGN \\
\hline $222758+003706$ & 336.992096 & 0.618294 & $48.766 \pm 0.240$ & $39.161 \pm 0.160$ & $51.889 \pm 0.155$ & $45.320 \pm 0.095$ & -0.30 & $83.75 \pm 0.10$ & $64.45 \pm 0.63$ & 18.1 & $\mathrm{nv}$ & $\ldots$ & $\ldots$ & QSO \\
\hline $223607+005259$ & 339.028010 & 0.883015 & $0.951 \pm 0.068$ & $0.941 \pm 0.056$ & $1.319 \pm 0.055$ & $0.940 \pm 0.053$ & -0.37 & $<0.50$ & $0.62 \pm 0.05$ & & $\ldots$ & & . & AGN \\
\hline $223624+004021$ & 339.099104 & 0.672614 & $0.886 \pm 0.071$ & $0.955 \pm 0.055$ & $0.595 \pm 0.060$ & $0.828 \pm 0.052$ & 0.43 & $<0.50$ & $0.57 \pm 0.07$ & 22.3 & $\ldots$ & $0.59^{\mathrm{a}}$ & 30.9 & AGN \\
\hline 224843-005459 & 342.178945 & -0.916381 & $1.644 \pm 0.085$ & $1.429 \pm 0.064$ & $1.778 \pm 0.085$ & $1.523 \pm 0.057$ & -0.27 & $<0.72$ & $0.69 \pm 0.07$ & $\ldots$ & $\ldots$ & $\ldots$ & $\ldots$ & AGN \\
\hline $224922+001805$ & 342.343018 & 0.301369 & $10.370 \pm 0.161$ & $10.029 \pm 0.137$ & $7.249 \pm 0.146$ & $9.759 \pm 0.059$ & 0.27 & $9.46 \pm 0.10$ & $11.51 \pm 0.06$ & $\ldots$ & $\ldots$ & $\ldots$ & $\ldots$ & AGN \\
\hline 225411-010651 & 343.544297 & -1.114119 & $0.644 \pm 0.063$ & $0.782 \pm 0.050$ & $0.300 \pm 0.056$ & $0.300 \pm 0.052$ & 0.85 & $<0.74$ & $<0.60$ & & $\ldots$ & $\ldots$ & $\ldots$ & AGN \\
\hline $230117-000204$ & 345.322041 & -0.034390 & $2.071 \pm 0.079$ & $2.600 \pm 0.062$ & $1.701 \pm 0.073$ & $1.946 \pm 0.054$ & 0.37 & $2.67 \pm 0.11$ & $2.09 \pm 0.07$ & 19.6 & $\mathrm{nv}$ & $0.28^{\mathrm{a}}$ & 30.6 & AGN \\
\hline $230133-002538$ & 345.387209 & -0.427294 & $0.676 \pm 0.082$ & $0.392 \pm 0.073$ & $0.876 \pm 0.079$ & $0.524 \pm 0.052$ & -0.80 & $<0.61$ & $0.34 \pm 0.06$ & $\ldots$ & $\ldots$ & $0.84^{\mathrm{a}}$ & 31.0 & AGN \\
\hline $230556-001652$ & 346.481658 & -0.281193 & $1.071 \pm 0.091$ & $0.900 \pm 0.074$ & $1.371 \pm 0.079$ & $1.031 \pm 0.064$ & -0.46 & $<0.51$ & $0.89 \pm 0.17$ & $\ldots$ & $\ldots$ & $\ldots$ & $\ldots$ & AGN \\
\hline 230636-002609 & 346.649268 & -0.435896 & $1.741 \pm 0.083$ & $1.150 \pm 0.074$ & $2.002 \pm 0.084$ & $1.607 \pm 0.062$ & -0.58 & $1.71 \pm 0.11$ & $1.48 \pm 0.09$ & $\ldots$ & $\ldots$ & $\ldots$ & $\ldots$ & AGN \\
\hline $230918+002642$ & 347.324430 & 0.445083 & $0.472 \pm 0.068$ & $0.350 \pm 0.055$ & $0.716 \pm 0.065$ & $0.433 \pm 0.065$ & -0.73 & $<0.50$ & $<0.39$ & $\ldots$ & $\ldots$ & $\ldots$ & $\ldots$ & AGN \\
\hline $231437+003844$ & 348.655518 & 0.645515 & $0.774 \pm 0.070$ & $0.805 \pm 0.053$ & $1.170 \pm 0.060$ & $1.026 \pm 0.090$ & -0.42 & $<0.53$ & $0.39 \pm 0.07$ & 24.9 & $\ldots$ & $\ldots$ & $\ldots$ & AGN \\
\hline $231444+004026$ & 348.685137 & 0.673960 & $0.233 \pm 0.060$ & $0.606 \pm 0.053$ & $0.222 \pm 0.055$ & $0.318 \pm 0.081$ & 0.89 & $<0.53$ & $0.34 \pm 0.06$ & 23.9 & $\ldots$ & $\ldots$ & $\ldots$ & AGN \\
\hline 231559-001205 & 348.994359 & -0.201415 & $4.176 \pm 0.105$ & $4.039 \pm 0.095$ & $5.282 \pm 0.098$ & $4.003 \pm 0.070$ & -0.31 & $4.90 \pm 0.10$ & $4.21 \pm 0.05$ & 23.1 & $\ldots$ & $\ldots$ & $\ldots$ & AGN \\
\hline $231746-005355$ & 349.441332 & -0.898619 & $1.045 \pm 0.081$ & $0.862 \pm 0.074$ & $1.284 \pm 0.085$ & $1.175 \pm 0.084$ & -0.44 & $<0.69$ & $0.44 \pm 0.05$ & - & $\ldots$ & & $\ldots$ & AGN \\
\hline 231942-004547 & 349.925567 & -0.763046 & $3.373 \pm 0.088$ & $4.311 \pm 0.076$ & $3.093 \pm 0.085$ & $3.372 \pm 0.074$ & 0.29 & $4.36 \pm 0.14$ & $3.89 \pm 0.06$ & 23.9 & $\ldots$ & $\ldots$ & $\ldots$ & AGN \\
\hline $232217+001252$ & 350.569589 & 0.214481 & $0.933 \pm 0.096$ & $0.700 \pm 0.078$ & $1.226 \pm 0.084$ & $1.081 \pm 0.060$ & -0.59 & $<0.49$ & $\ldots$ & & $\ldots$ & $\ldots$ & $\ldots$ & AGN \\
\hline $232226+010357$ & 350.606463 & 1.065718 & $3.082 \pm 0.193$ & $2.164 \pm 0.131$ & $3.145 \pm 0.168$ & $3.577 \pm 0.093$ & -0.42 & $4.96 \pm 0.13$ & & 17.4 & $\ldots$ & 0.12 & 30.0 & AGN \\
\hline 232634-010513 & 351.642405 & -1.086815 & $2.481 \pm 0.129$ & $1.565 \pm 0.121$ & $2.239 \pm 0.128$ & $2.241 \pm 0.086$ & -0.40 & $1.50 \pm 0.15$ & & 23.4 & $\ldots$ & $0.79^{\mathrm{a}}$ & 31.5 & AGN \\
\hline $232656-000438$ & 351.734689 & -0.077164 & $1.737 \pm 0.060$ & $1.477 \pm 0.056$ & $2.050 \pm 0.055$ & $1.549 \pm 0.065$ & -0.37 & $1.09 \pm 0.10$ & $\ldots$ & 24.1 & $\ldots$ & $\ldots$ & $\ldots$ & AGN \\
\hline $232723-000507$ & 351.843984 & -0.085213 & $1.373 \pm 0.071$ & $1.796 \pm 0.062$ & $1.316 \pm 0.062$ & $1.478 \pm 0.073$ & 0.27 & $1.25 \pm 0.10$ & & $\ldots$ & $\ldots$ & $\ldots$ & & AGN \\
\hline 232933-004002 & 352.386845 & -0.667239 & $8.785 \pm 0.165$ & $9.301 \pm 0.143$ & $6.812 \pm 0.149$ & $12.592 \pm 0.086$ & 0.26 & $6.93 \pm 0.14$ & & 22.4 & $\ldots$ & $\ldots$ & & AGN \\
\hline $233106+002607$ & 352.774153 & 0.435175 & $0.841 \pm 0.054$ & $0.644 \pm 0.044$ & $0.922 \pm 0.047$ & $0.648 \pm 0.057$ & -0.40 & $1.00 \pm 0.11$ & $\ldots$ & 22.2 & $\ldots$ & 0.74 & 31.1 & AGN \\
\hline \multicolumn{15}{|c|}{ Timescale $<1$ month } \\
\hline $220127+001402$ & 330.360610 & 0.233772 & $1.076 \pm 0.096$ & $0.448 \pm 0.071$ & $0.480 \pm 0.069$ & $0.660 \pm 0.053$ & 0.82 & $<0.49$ & & 23.9 & $\ldots$ & $\ldots$ & $\ldots$ & AGN \\
\hline 220456-000147 & 331.232587 & -0.029821 & $4.999 \pm 0.169$ & $6.578 \pm 0.096$ & $5.969 \pm 0.125$ & $6.192 \pm 0.076$ & -0.27 & $2.29 \pm 0.11$ & $2.45 \pm 0.26$ & & $\ldots$ & $\ldots$ & $\ldots$ & AGN \\
\hline 220643-003102 & 331.680375 & -0.517180 & $131.189 \pm 0.699$ & $194.029 \pm 0.340$ & $151.652 \pm 0.298$ & $134.492 \pm 0.182$ & -0.39 & $122.89 \pm 0.13$ & $73.21 \pm 0.76$ & 19.5 & $\mathrm{~V}$ & $\ldots$ & $\ldots$ & QSO \\
\hline 220910-001601 & 332.293354 & -0.267023 & $6.690 \pm 0.166$ & $9.455 \pm 0.120$ & $7.796 \pm 0.137$ & $8.323 \pm 0.054$ & -0.34 & $8.39 \pm 0.10$ & $5.78 \pm 0.06$ & 19.5 & $\mathrm{nv}$ & 1.11 & 32.4 & QSO \\
\hline $221308-010837$ & 333.284558 & -1.143507 & $0.921 \pm 0.075$ & $0.376 \pm 0.057$ & $0.614 \pm 0.067$ & $0.711 \pm 0.059$ & 0.84 & $<0.72$ & $0.95 \pm 0.05$ & 23.2 & $\ldots$ & & & AGN \\
\hline $221350-011130$ & 333.460186 & -1.191627 & $1.540 \pm 0.120$ & $0.835 \pm 0.086$ & $1.083 \pm 0.101$ & $1.139 \pm 0.052$ & 0.59 & $<0.71$ & $<0.28$ & 20.0 & $\ldots$ & 1.96 & 32.0 & QSO \\
\hline $221959+011045$ & 334.994454 & 1.179204 & $4.827 \pm 0.104$ & $3.679 \pm 0.081$ & $3.710 \pm 0.107$ & $4.981 \pm 0.052$ & 0.27 & $4.62 \pm 0.14$ & $3.42 \pm 0.07$ & 21.0 & $\ldots$ & 0.84 & 31.9 & QSO \\
\hline 222123-002509 & 335.345654 & -0.419074 & $3.255 \pm 0.145$ & $2.235 \pm 0.107$ & $2.724 \pm 0.119$ & $2.674 \pm 0.080$ & 0.37 & $3.62 \pm 0.10$ & $2.70 \pm 0.10$ & 23.4 & $\ldots$ & $\ldots$ & $\ldots$ & AGN \\
\hline $222942+003556$ & 337.423504 & 0.598817 & $3.266 \pm 0.089$ & $2.401 \pm 0.070$ & $2.772 \pm 0.073$ & $2.367 \pm 0.051$ & 0.31 & $2.63 \pm 0.11$ & $1.97 \pm 0.06$ & 24.5 & $\ldots$ & $\ldots$ & $\ldots$ & AGN \\
\hline 223317-005009 & 338.321408 & -0.835723 & $1.977 \pm 0.093$ & $1.344 \pm 0.068$ & $1.448 \pm 0.066$ & $1.344 \pm 0.066$ & 0.38 & $1.80 \pm 0.14$ & $1.27 \pm 0.06$ & $\ldots$ & $\ldots$ & $\ldots$ & & AGN \\
\hline $223612+001007$ & 339.050505 & 0.168559 & $0.804 \pm 0.086$ & $0.271 \pm 0.071$ & $0.179 \pm 0.073$ & $0.098 \pm 0.057$ & 0.99 & $<0.52$ & $<0.39$ & 9.7 & $\ldots$ & $\ldots$ & $\ldots$ & RS CVn \\
\hline $224036+010852$ & 340.150070 & 1.147826 & $2.005 \pm 0.070$ & $2.729 \pm 0.054$ & $2.411 \pm 0.056$ & $1.686 \pm 0.057$ & -0.31 & $2.13 \pm 0.14$ & $1.41 \pm 0.06$ & 21.0 & $\ldots$ & 2.99 & 32.7 & QSO \\
\hline 224448-000619 & 341.200443 & -0.105397 & $8.637 \pm 0.086$ & $6.604 \pm 0.074$ & $5.485 \pm 0.079$ & $6.716 \pm 0.064$ & 0.27 & $6.90 \pm 0.10$ & $7.21 \pm 0.07$ & 18.9 & nv & $\ldots$ & & QSO \\
\hline $224657+005240$ & 341.737102 & 0.877754 & $2.641 \pm 0.063$ & $1.928 \pm 0.057$ & $1.702 \pm 0.071$ & $1.982 \pm 0.054$ & 0.31 & $1.40 \pm 0.13$ & $0.75 \pm 0.06$ & $\ldots$ & $\ldots$ & $\ldots$ & & AGN \\
\hline 225649-005401 & 344.205944 & -0.900221 & $1.935 \pm 0.086$ & $1.397 \pm 0.074$ & $1.364 \pm 0.077$ & $1.480 \pm 0.059$ & 0.32 & $1.63 \pm 0.14$ & $1.74 \pm 0.06$ & & $\ldots$ & & $\ldots$ & AGN \\
\hline 225707-010238 & 344.280373 & -1.043805 & $0.602 \pm 0.073$ & $1.218 \pm 0.069$ & $0.914 \pm 0.065$ & $1.192 \pm 0.056$ & -0.68 & $<0.74$ & $<0.39$ & 20.1 & $\mathrm{nv}$ & 1.56 & 31.8 & QSO \\
\hline
\end{tabular}


Table 3

(Continued)

\begin{tabular}{|c|c|c|c|c|c|c|c|c|c|c|c|c|c|c|}
\hline VTC & $\begin{array}{c}\alpha_{\mathrm{J} 2000} \\
(\mathrm{deg})\end{array}$ & $\begin{array}{r}\delta_{\mathrm{J} 2000} \\
(\mathrm{deg})\end{array}$ & $\begin{array}{c}S_{\mathrm{E} 1} \\
(\mathrm{mJy})\end{array}$ & $\begin{array}{c}S_{\mathrm{E} 2} \\
(\mathrm{mJy})\end{array}$ & $\begin{array}{c}S_{\mathrm{E} 3} \\
(\mathrm{mJy})\end{array}$ & $\begin{array}{c}S_{\mathrm{E} 4} \\
(\mathrm{mJy})\end{array}$ & $m$ & $\begin{array}{r}\text { FIRST } \\
(\mathrm{mJy})\end{array}$ & $\begin{array}{l}\text { Hodge et al. (2011) } \\
(\mathrm{mJy})\end{array}$ & $\begin{array}{l}r_{\mathrm{SDSS}} \\
(\mathrm{mag})\end{array}$ & PTF & $z_{\text {spec }}$ & $\begin{array}{c}\log \bar{L}_{\nu} \\
\left(\operatorname{erg~cm}^{-2} \mathrm{~s}^{-1}\right)\end{array}$ & Identification \\
\hline $230236+005739$ & 345.647977 & 0.960878 & $4.146 \pm 0.142$ & $3.165 \pm 0.114$ & $3.344 \pm 0.144$ & $4.485 \pm 0.054$ & 0.27 & $3.43 \pm 0.11$ & $4.92 \pm 0.06$ & & $\ldots$ & & & AGN \\
\hline 230803-000145 & 347.012572 & -0.029111 & $0.376 \pm 0.058$ & $0.698 \pm 0.053$ & $0.453 \pm 0.056$ & $0.351 \pm 0.062$ & -0.60 & $<0.52$ & $<0.29$ & 18.5 & $\mathrm{nv}$ & 0.15 & 29.5 & AGN \\
\hline $230810+002117$ & 347.039702 & 0.354644 & $1.200 \pm 0.063$ & $1.771 \pm 0.055$ & $1.322 \pm 0.061$ & $0.903 \pm 0.078$ & -0.38 & $1.33 \pm 0.11$ & $1.03 \pm 0.06$ & 21.1 & $\mathrm{nv}$ & $0.40^{\mathrm{a}}$ & 30.7 & AGN \\
\hline $231242+002451$ & 348.174063 & 0.414044 & $4.347 \pm 0.095$ & $3.198 \pm 0.079$ & $3.767 \pm 0.083$ & $2.386 \pm 0.062$ & 0.30 & $1.19 \pm 0.10$ & $0.86 \pm 0.06$ & 18.7 & $\mathrm{nv}$ & 1.90 & 32.5 & QSO \\
\hline 231334-001645 & 348.390098 & -0.279113 & $2.343 \pm 0.061$ & $1.440 \pm 0.054$ & $1.353 \pm 0.061$ & $1.657 \pm 0.064$ & 0.48 & $1.87 \pm 0.10$ & $2.20 \pm 0.05$ & 22.6 & $\ldots$ & 0.74 & 31.4 & AGN \\
\hline $231455+002456$ & 348.728453 & 0.415556 & $1.177 \pm 0.071$ & $0.757 \pm 0.064$ & $0.873 \pm 0.064$ & $0.855 \pm 0.075$ & 0.43 & $0.96 \pm 0.10$ & $0.81 \pm 0.06$ & 24.8 & $\ldots$ & & & AGN \\
\hline 232125-004845 & 350.352737 & -0.812522 & $2.527 \pm 0.072$ & $1.829 \pm 0.065$ & $1.822 \pm 0.068$ & $1.905 \pm 0.071$ & 0.32 & $1.94 \pm 0.14$ & $\ldots$ & 21.8 & $\ldots$ & $0.58^{\mathrm{a}}$ & 31.2 & AGN \\
\hline 232526-003623 & 351.359745 & -0.606393 & $2.039 \pm 0.092$ & $2.672 \pm 0.075$ & $2.098 \pm 0.078$ & $2.535 \pm 0.076$ & -0.27 & $3.26 \pm 0.13$ & $\ldots$ & 21.4 & $\mathrm{nv}$ & 0.63 & 31.4 & AGN \\
\hline $232548-011134$ & 351.451958 & -1.192766 & $5.404 \pm 0.148$ & $7.212 \pm 0.145$ & $5.776 \pm 0.140$ & $5.925 \pm 0.083$ & -0.29 & $6.21 \pm 0.14$ & $\ldots$ & 20.7 & $\ldots$ & 0.46 & 31.5 & AGN \\
\hline \multicolumn{15}{|c|}{ Timescale $<1.5 \mathrm{yr}$} \\
\hline $215701+005124$ & 329.252431 & 0.856537 & $0.923 \pm 0.074$ & $1.142 \pm 0.052$ & $0.811 \pm 0.059$ & $0.764 \pm 0.043$ & 0.40 & $1.06 \pm 0.10$ & $\ldots$ & 22.9 & & $0.57^{\mathrm{a}}$ & 30.9 & AGN \\
\hline $215732+010748$ & 329.384654 & 1.130011 & $1.219 \pm 0.099$ & $1.195 \pm 0.060$ & $1.048 \pm 0.065$ & $0.806 \pm 0.053$ & 0.39 & $<0.78$ & $\ldots$ & 19.1 & $\mathrm{nv}$ & 0.30 & 30.4 & AGN \\
\hline $215929+004723$ & 329.872536 & 0.789814 & $1.777 \pm 0.118$ & $1.586 \pm 0.095$ & $1.500 \pm 0.087$ & $2.091 \pm 0.043$ & -0.27 & $1.52 \pm 0.11$ & $\ldots$ & $\ldots$ & $\ldots$ & $\ldots$ & $\ldots$ & AGN \\
\hline $215951+010041$ & 329.963762 & 1.011278 & $1.405 \pm 0.102$ & $1.375 \pm 0.075$ & $1.300 \pm 0.074$ & $2.005 \pm 0.044$ & -0.37 & $2.03 \pm 0.12$ & $\ldots$ & $\ldots$ & $\ldots$ & $\ldots$ & $\ldots$ & AGN \\
\hline $220005+002309$ & 330.022192 & 0.385698 & $1.357 \pm 0.068$ & $1.316 \pm 0.054$ & $1.217 \pm 0.066$ & $0.962 \pm 0.056$ & 0.31 & $<0.51$ & $\ldots$ & & $\ldots$ & $\ldots$ & $\ldots$ & AGN \\
\hline 220017-000134 & 330.072365 & -0.026093 & $5.912 \pm 0.100$ & $5.995 \pm 0.080$ & $4.965 \pm 0.087$ & $4.612 \pm 0.067$ & 0.26 & $7.06 \pm 0.09$ & $\ldots$ & 19.8 & $\mathrm{nv}$ & 0.61 & 31.7 & QSO \\
\hline $220109+010124$ & 330.288600 & 1.023416 & $12.343 \pm 0.147$ & $11.386 \pm 0.107$ & $11.627 \pm 0.096$ & $15.358 \pm 0.049$ & -0.30 & $37.55 \pm 0.14$ & $\ldots$ & $\ldots$ & $\ldots$ & $\ldots$ & $\ldots$ & AGN \\
\hline $220110+002547$ & 330.293140 & 0.429823 & $2.504 \pm 0.077$ & $2.715 \pm 0.058$ & $2.335 \pm 0.056$ & $2.049 \pm 0.056$ & 0.28 & $3.76 \pm 0.11$ & $\ldots$ & $\ldots$ & $\ldots$ & $\ldots$ & $\ldots$ & AGN \\
\hline $220221+001114$ & 330.587782 & 0.187335 & $1.438 \pm 0.078$ & $1.623 \pm 0.065$ & $1.616 \pm 0.060$ & $1.212 \pm 0.045$ & 0.29 & $2.00 \pm 0.11$ & $\ldots$ & 24.2 & $\ldots$ & $\ldots$ & $\ldots$ & AGN \\
\hline $220445+005129$ & 331.187680 & 0.857985 & $3.041 \pm 0.089$ & $2.913 \pm 0.066$ & $2.641 \pm 0.069$ & $3.801 \pm 0.050$ & -0.26 & $4.76 \pm 0.13$ & $3.10 \pm 0.15$ & & $\ldots$ & & & AGN \\
\hline $220708+010126$ & 331.784654 & 1.023799 & $7.029 \pm 0.173$ & $6.286 \pm 0.122$ & $6.481 \pm 0.131$ & $8.313 \pm 0.053$ & -0.28 & $15.68 \pm 0.13$ & $10.72 \pm 0.06$ & 18.8 & $\mathrm{nv}$ & 2.91 & 33.2 & QSO \\
\hline $220804+000556$ & 332.018501 & 0.098983 & $6.253 \pm 0.163$ & $5.422 \pm 0.118$ & $5.585 \pm 0.128$ & $7.053 \pm 0.058$ & -0.26 & $10.06 \pm 0.11$ & $7.99 \pm 0.07$ & 23.2 & $\ldots$ & $\ldots$ & $\ldots$ & AGN \\
\hline $220904+004607$ & 332.266020 & 0.768647 & $1.317 \pm 0.160$ & $1.712 \pm 0.133$ & $1.042 \pm 0.143$ & $1.008 \pm 0.043$ & 0.52 & $<0.51$ & $1.00 \pm 0.05$ & & $\ldots$ & $\ldots$ & $\ldots$ & AGN \\
\hline $221160-003139$ & 332.998688 & -0.527367 & $1.362 \pm 0.077$ & $1.073 \pm 0.057$ & $1.174 \pm 0.061$ & $1.414 \pm 0.051$ & -0.27 & $2.10 \pm 0.10$ & $2.12 \pm 0.05$ & 23.9 & & & $\ldots$ & AGN \\
\hline 221257-005711 & 333.235700 & -0.953020 & $1.897 \pm 0.160$ & $1.319 \pm 0.125$ & $1.807 \pm 0.136$ & $2.194 \pm 0.055$ & -0.50 & $3.51 \pm 0.15$ & $2.83 \pm 0.06$ & & & & $\ldots$ & AGN \\
\hline $221548-001031$ & 333.948344 & -0.175409 & $0.882 \pm 0.102$ & $0.755 \pm 0.083$ & $1.083 \pm 0.081$ & $1.283 \pm 0.051$ & -0.52 & $1.30 \pm 0.10$ & $1.36 \pm 0.06$ & 22.6 & $\ldots$ & $0.54^{\mathrm{a}}$ & 30.9 & AGN \\
\hline 221609-005708 & 334.037121 & -0.952234 & $9.000 \pm 0.158$ & $7.481 \pm 0.149$ & $7.805 \pm 0.149$ & $11.971 \pm 0.064$ & -0.46 & $6.56 \pm 0.15$ & $5.35 \pm 0.05$ & 17.5 & nv & 2.40 & 33.2 & QSO \\
\hline 221642-004904 & 334.174200 & -0.817899 & $1.734 \pm 0.069$ & $1.828 \pm 0.055$ & $2.074 \pm 0.062$ & $2.383 \pm 0.054$ & -0.26 & $1.24 \pm 0.15$ & $2.46 \pm 0.06$ & 22.8 & & $0.69^{\mathrm{a}}$ & 31.4 & AGN \\
\hline 222038-001209 & 335.158511 & -0.202623 & $1.916 \pm 0.205$ & $1.596 \pm 0.157$ & $1.922 \pm 0.158$ & $2.574 \pm 0.047$ & -0.47 & $10.26 \pm 0.11$ & $7.29 \pm 0.07$ & & $\ldots$ & & & AGN \\
\hline 222109-001940 & 335.286275 & -0.327789 & $0.959 \pm 0.125$ & $0.907 \pm 0.095$ & $0.892 \pm 0.105$ & $1.563 \pm 0.061$ & -0.53 & $1.59 \pm 0.10$ & $1.20 \pm 0.08$ & 22.4 & $\ldots$ & 2.36 & 32.2 & QSO \\
\hline 222127-001530 & 335.362235 & -0.258239 & $1.779 \pm 0.121$ & $1.720 \pm 0.085$ & $1.558 \pm 0.085$ & $2.316 \pm 0.055$ & -0.30 & $2.06 \pm 0.11$ & $1.67 \pm 0.10$ & 23.2 & $\ldots$ & $0.46^{\mathrm{a}}$ & 31.0 & AGN \\
\hline 222201-005008 & 335.505891 & -0.835608 & $8.209 \pm 0.141$ & $7.158 \pm 0.113$ & $8.512 \pm 0.109$ & $12.122 \pm 0.070$ & -0.51 & $5.56 \pm 0.15$ & $4.02 \pm 0.07$ & 20.1 & nv & $0.33^{\mathrm{a}}$ & 31.4 & AGN \\
\hline $222359+011148$ & 335.997306 & 1.196745 & $1.044 \pm 0.116$ & $1.006 \pm 0.087$ & $1.118 \pm 0.084$ & $1.478 \pm 0.054$ & -0.38 & $1.70 \pm 0.14$ & $0.75 \pm 0.15$ & 22.4 & & & & AGN \\
\hline $222443+002858$ & 336.180822 & 0.482741 & $1.718 \pm 0.109$ & $1.832 \pm 0.075$ & $1.917 \pm 0.084$ & $2.447 \pm 0.044$ & -0.29 & $2.12 \pm 0.11$ & $1.52 \pm 0.06$ & 19.5 & nv & $0.26^{\mathrm{a}}$ & 30.5 & AGN \\
\hline 222524-001837 & 336.348824 & -0.310389 & $0.934 \pm 0.080$ & $1.058 \pm 0.056$ & $0.862 \pm 0.060$ & $0.704 \pm 0.055$ & 0.40 & $<0.55$ & $1.11 \pm 0.06$ & $\ldots$ & $\ldots$ & $\ldots$ & $\ldots$ & AGN \\
\hline $222546+004038$ & 336.439976 & 0.677156 & $1.572 \pm 0.069$ & $1.607 \pm 0.060$ & $1.667 \pm 0.060$ & $1.146 \pm 0.060$ & 0.34 & $2.18 \pm 0.10$ & $2.92 \pm 0.07$ & & $\ldots$ & & & AGN \\
\hline 222605-010441 & 336.521610 & -1.078109 & $4.218 \pm 0.075$ & $4.657 \pm 0.053$ & $4.261 \pm 0.054$ & $3.465 \pm 0.055$ & 0.29 & $5.22 \pm 0.15$ & $4.06 \pm 0.06$ & 22.9 & $\ldots$ & $0.42^{\mathrm{a}}$ & 31.3 & AGN \\
\hline 222630-001248 & 336.625294 & -0.213342 & $4.690 \pm 0.134$ & $4.418 \pm 0.104$ & $4.561 \pm 0.121$ & $5.855 \pm 0.052$ & -0.28 & $10.75 \pm 0.11$ & $7.66 \pm 0.07$ & & $\ldots$ & & & AGN \\
\hline $222647+005211$ & 336.694041 & 0.869751 & $359.852 \pm 0.879$ & $324.569 \pm 0.639$ & $362.536 \pm 0.610$ & $433.361 \pm 0.246$ & -0.29 & $617.48 \pm 0.10$ & $285.28 \pm 5.57$ & 21.3 & $\mathrm{nv}$ & 2.26 & 34.7 & QSO \\
\hline $222704+011055$ & 336.766725 & 1.182021 & $1.665 \pm 0.150$ & $1.488 \pm 0.098$ & $1.302 \pm 0.090$ & $2.291 \pm 0.065$ & -0.42 & $1.11 \pm 0.14$ & $<3.06$ & 17.4 & & $0.06^{\mathrm{a}}$ & 29.1 & AGN \\
\hline $222907+001908$ & 337.277758 & 0.318936 & $1.107 \pm 0.098$ & $0.918 \pm 0.086$ & $1.031 \pm 0.079$ & $1.423 \pm 0.048$ & -0.43 & $1.51 \pm 0.11$ & $0.91 \pm 0.06$ & & $\mathrm{nv}$ & 1.80 & 32.0 & QSO \\
\hline 222930-000845 & 337.373252 & -0.145791 & $3.183 \pm 0.141$ & $3.171 \pm 0.106$ & $3.020 \pm 0.100$ & $4.163 \pm 0.059$ & -0.27 & $4.40 \pm 0.11$ & $2.66 \pm 0.06$ & 22.9 & & $0.56^{\mathrm{a}}$ & 31.5 & AGN \\
\hline $223024+004334$ & 337.598552 & 0.726077 & $1.417 \pm 0.082$ & $1.180 \pm 0.069$ & $1.210 \pm 0.066$ & $1.586 \pm 0.047$ & -0.29 & $1.44 \pm 0.10$ & $0.67 \pm 0.08$ & 18.3 & $\mathrm{nv}$ & 0.13 & 29.7 & AGN \\
\hline $223047+003160$ & 337.694749 & 0.533246 & $2.088 \pm 0.153$ & $1.877 \pm 0.122$ & $1.881 \pm 0.126$ & $2.860 \pm 0.047$ & -0.41 & $3.93 \pm 0.11$ & $3.70 \pm 0.06$ & 17.4 & nv & 0.09 & 29.7 & AGN \\
\hline $223140+002305$ & 337.917233 & 0.384585 & $0.775 \pm 0.092$ & $0.641 \pm 0.071$ & $0.616 \pm 0.068$ & $1.125 \pm 0.057$ & -0.55 & $1.83 \pm 0.10$ & $1.36 \pm 0.06$ & & & & $\ldots$ & AGN \\
\hline $223225+003431$ & 338.103745 & 0.575289 & $1.785 \pm 0.086$ & $1.871 \pm 0.071$ & $1.385 \pm 0.067$ & $1.370 \pm 0.048$ & 0.31 & $2.19 \pm 0.11$ & $2.13 \pm 0.06$ & & $\ldots$ & $\ldots$ & $\ldots$ & AGN \\
\hline $223409+010618$ & 338.537649 & 1.105054 & $14.140 \pm 0.123$ & $16.928 \pm 0.104$ & $16.556 \pm 0.110$ & $22.451 \pm 0.069$ & -0.28 & $27.15 \pm 0.13$ & $24.37 \pm 0.24$ & 22.0 & $\ldots$ & & & AGN \\
\hline 223516-005849 & 338.817742 & -0.980367 & $3.230 \pm 0.144$ & $3.480 \pm 0.110$ & $3.026 \pm 0.114$ & $4.715 \pm 0.059$ & -0.30 & $2.91 \pm 0.15$ & $4.54 \pm 0.06$ & 19.1 & $\mathrm{nv}$ & 1.18 & 32.2 & QSO \\
\hline $223908+003232$ & 339.784225 & 0.542353 & $4.063 \pm 0.249$ & $4.083 \pm 0.167$ & $4.267 \pm 0.184$ & $5.843 \pm 0.058$ & -0.35 & $3.83 \pm 0.10$ & $4.01 \pm 0.08$ & 19.3 & $\mathrm{nv}$ & $0.24^{\mathrm{a}}$ & 30.9 & AGN \\
\hline 224023-003555 & 340.096938 & -0.598702 & $2.583 \pm 0.100$ & $2.467 \pm 0.079$ & $2.953 \pm 0.093$ & $3.737 \pm 0.069$ & -0.41 & $5.67 \pm 0.13$ & $3.17 \pm 0.08$ & 18.9 & $\mathrm{nv}$ & 1.16 & 32.0 & QSO \\
\hline 224628-001214 & 341.615430 & -0.203797 & $56.423 \pm 0.343$ & $56.155 \pm 0.291$ & $55.536 \pm 0.307$ & $73.059 \pm 0.112$ & -0.26 & $70.42 \pm 0.10$ & $100.88 \pm 0.65$ & 21.8 & $\mathrm{nv}$ & $0.55^{\mathrm{a}}$ & 32.7 & AGN \\
\hline $224730+000006$ & 341.875813 & 0.001783 & $281.818 \pm 1.200$ & $272.963 \pm 0.769$ & $260.412 \pm 1.024$ & $545.119 \pm 0.403$ & -0.67 & $322.29 \pm 0.10$ & $397.62 \pm 2.48$ & 18.1 & $\mathrm{~V}$ & 0.97 & 34.1 & QSO \\
\hline $224733+010817$ & 341.885647 & 1.138013 & $1.415 \pm 0.061$ & $1.364 \pm 0.053$ & $1.202 \pm 0.065$ & $0.973 \pm 0.054$ & 0.34 & $1.78 \pm 0.14$ & $0.86 \pm 0.09$ & & $\ldots$ & & & AGN \\
\hline $224803+003959$ & 342.013923 & 0.666301 & $7.771 \pm 0.131$ & $6.594 \pm 0.099$ & $6.750 \pm 0.111$ & $9.238 \pm 0.068$ & -0.33 & $11.93 \pm 0.11$ & $8.39 \pm 0.06$ & 22.3 & $\ldots$ & $0.82^{\mathrm{a}}$ & 32.2 & AGN \\
\hline $225103+000156$ & 342.760572 & 0.032354 & $2.359 \pm 0.126$ & $2.126 \pm 0.114$ & $2.042 \pm 0.119$ & $3.040 \pm 0.060$ & -0.35 & $5.33 \pm 0.10$ & $3.64 \pm 0.06$ & 23.3 & $\ldots$ & & & AGN \\
\hline 225307-010950 & 343.277710 & -1.163845 & $2.320 \pm 0.079$ & $2.124 \pm 0.060$ & $2.011 \pm 0.071$ & $1.464 \pm 0.053$ & 0.37 & $1.39 \pm 0.14$ & $1.73 \pm 0.07$ & 20.5 & $\mathrm{nv}$ & 0.34 & 30.7 & AGN \\
\hline
\end{tabular}


Table 3

(Continued)

\begin{tabular}{|c|c|c|c|c|c|c|c|c|c|c|c|c|c|c|}
\hline VTC & $\begin{array}{l}\alpha_{\text {J22000 }} \\
(\operatorname{deg})\end{array}$ & $\begin{array}{c}\delta_{12000} \\
(\mathrm{deg})\end{array}$ & $\begin{array}{l}S_{\mathrm{E} 1} \\
(\mathrm{mJy})\end{array}$ & $\underset{(\mathrm{mJy})}{S_{\mathrm{E} 2}}$ & $\underset{(\mathrm{EJy})}{S_{\mathrm{E} 3}}$ & $\underset{(\mathrm{mJy})}{S_{\mathrm{E} 4}}$ & $m$ & $\begin{array}{c}\text { FIRST } \\
(\mathrm{mJy})\end{array}$ & $\begin{array}{l}\text { Hodge et al. (2011) } \\
\text { (mJy) }\end{array}$ & $\begin{array}{l}r_{\text {SDSS }} \\
\text { (mag) }\end{array}$ & PTF & $z_{\text {spec }}$ & $\begin{array}{c}\log \bar{L}_{\nu} \\
\left(\operatorname{erg} \mathrm{cm}^{-2} \mathrm{~s}^{-1}\right)\end{array}$ & Identification \\
\hline 225438-001641 & 343.658007 & -0.277990 & $3.564 \pm 0.080$ & $3.759 \pm 0.072$ & $3.375 \pm 0.077$ & $2.759 \pm 0.074$ & 0.31 & $2.92 \pm 0.11$ & $3.52 \pm 0.09$ & 22.0 & $\ldots$ & $0.38^{\mathrm{a}}$ & 31.1 & AGN \\
\hline $225510+002526$ & 343.791849 & 0.423809 & $0.548 \pm 0.067$ & $0.762 \pm 0.056$ & $0.495 \pm 0.060$ & $0.401 \pm 0.046$ & 0.62 & $<0.53$ & $0.42 \pm 0.07$ & & $\ldots$ & & & AGN \\
\hline 225525-000956 & 343.854026 & -0.165458 & $4.053 \pm 0.128$ & $3.776 \pm 0.104$ & $3.613 \pm 0.112$ & $5.175 \pm 0.081$ & -0.31 & $6.19 \pm 0.10$ & $4.77 \pm 0.12$ & 22.6 & $\ldots$ & $0.71^{\mathrm{a}}$ & 31.8 & AGN \\
\hline $225621-004110$ & 344.085666 & -0.686069 & $0.982 \pm 0.107$ & $1.318 \pm 0.091$ & $0.960 \pm 0.098$ & $0.866 \pm 0.050$ & 0.41 & $1.22 \pm 0.15$ & $0.94 \pm 0.07$ & 21.5 & $\mathrm{nv}$ & 0.56 & 30.9 & AGN \\
\hline $225934+010821$ & 344.891408 & 1.139151 & $0.742 \pm 0.060$ & $0.966 \pm 0.052$ & $0.984 \pm 0.052$ & $0.610 \pm 0.046$ & 0.45 & $<0.69$ & $<0.34$ & 23.7 & $\ldots$ & $\ldots$ & $\ldots$ & AGN \\
\hline 225936-003356 & 344.898924 & -0.565593 & $2.109 \pm 0.128$ & $1.989 \pm 0.110$ & $1.844 \pm 0.124$ & $2.683 \pm 0.066$ & -0.30 & $5.78 \pm 0.12$ & $4.94 \pm 0.06$ & $\ldots$ & $\ldots$ & $\ldots$ & $\ldots$ & AGN \\
\hline $230112-002112$ & 345.299432 & -0.353462 & $1.611 \pm 0.108$ & $1.523 \pm 0.084$ & $1.484 \pm 0.087$ & $2.122 \pm 0.070$ & -0.33 & $5.52 \pm 0.11$ & $2.97 \pm 0.06$ & $\ldots$ & $\ldots$ & $\ldots$ & $\ldots$ & AGN \\
\hline 230132-010319 & 345.382529 & -1.055362 & $2.802 \pm 0.106$ & $2.772 \pm 0.091$ & $2.890 \pm 0.106$ & $4.338 \pm 0.056$ & -0.44 & $11.78 \pm 0.14$ & $7.11 \pm 0.07$ & $\ldots$ & $\ldots$ & $\ldots$ & $\ldots$ & AGN \\
\hline $230158+000352$ & 345.490838 & 0.064493 & $7.164 \pm 0.143$ & $5.936 \pm 0.123$ & $6.574 \pm 0.154$ & $12.024 \pm 0.059$ & -0.68 & $5.38 \pm 0.11$ & $5.02 \pm 0.07$ & 23.1 & $\ldots$ & & & AGN \\
\hline 230218-005817 & 345.576721 & -0.971495 & $2.119 \pm 0.152$ & $2.245 \pm 0.122$ & $2.255 \pm 0.126$ & $2.936 \pm 0.063$ & -0.27 & $14.39 \pm 0.15$ & $10.93 \pm 0.09$ & & & $0.91^{\mathrm{a}}$ & 31.8 & AGN \\
\hline 230334-004006 & 345.890039 & -0.668195 & $3.817 \pm 0.155$ & $3.400 \pm 0.120$ & $3.223 \pm 0.140$ & $4.479 \pm 0.070$ & -0.27 & $7.41 \pm 0.14$ & $5.79 \pm 0.07$ & 22.0 & $\ldots$ & $0.70^{\mathrm{a}}$ & 31.7 & AGN \\
\hline 230423-000417 & 346.096113 & -0.071504 & $4.115 \pm 0.066$ & $3.415 \pm 0.065$ & $3.576 \pm 0.066$ & $2.608 \pm 0.064$ & 0.27 & $2.44 \pm 0.10$ & $3.84 \pm 0.08$ & 19.7 & nv & 1.05 & 32.0 & QSO \\
\hline $230748+002213$ & 346.950170 & 0.370314 & $4.502 \pm 0.083$ & $4.657 \pm 0.069$ & $4.229 \pm 0.077$ & $2.940 \pm 0.103$ & 0.45 & $7.68 \pm 0.10$ & $6.26 \pm 0.06$ & 22.4 & & $0.71^{\mathrm{a}}$ & 31.8 & AGN \\
\hline $230847+010904$ & 347.196936 & 1.151171 & $1.371 \pm 0.063$ & $1.303 \pm 0.054$ & $1.295 \pm 0.059$ & $0.872 \pm 0.064$ & 0.40 & $2.44 \pm 0.14$ & $1.52 \pm 0.14$ & $\ldots$ & $\ldots$ & $\ldots$ & $\ldots$ & AGN \\
\hline $231014+002531$ & 347.559929 & 0.425187 & $17.643 \pm 0.098$ & $18.021 \pm 0.074$ & $16.694 \pm 0.080$ & $13.110 \pm 0.117$ & 0.32 & $65.78 \pm 0.11$ & $37.53 \pm 0.43$ & & $\ldots$ & $\ldots$ & $\ldots$ & AGN \\
\hline $231210-003135$ & 348.042870 & -0.526485 & $2.677 \pm 0.073$ & $2.661 \pm 0.070$ & $2.580 \pm 0.079$ & $1.596 \pm 0.098$ & 0.50 & $5.86 \pm 0.10$ & $5.50 \pm 0.07$ & 23.3 & $\ldots$ & $\ldots$ & $\ldots$ & AGN \\
\hline $231517+002630$ & 348.822686 & 0.441566 & $3.696 \pm 0.080$ & $3.709 \pm 0.065$ & $3.481 \pm 0.065$ & $2.853 \pm 0.100$ & 0.26 & $8.88 \pm 0.10$ & $9.35 \pm 0.06$ & $\ldots$ & & & $\ldots$ & AGN \\
\hline 231537-003726 & 348.902796 & -0.623911 & $1.678 \pm 0.134$ & $1.286 \pm 0.126$ & $1.564 \pm 0.127$ & $3.021 \pm 0.091$ & -0.81 & $2.05 \pm 0.14$ & $1.20 \pm 0.06$ & & $\mathrm{nv}$ & & & AGN \\
\hline $231542+002937$ & 348.923546 & 0.493578 & $12.632 \pm 0.170$ & $13.768 \pm 0.137$ & $12.830 \pm 0.135$ & $9.258 \pm 0.120$ & 0.39 & $17.92 \pm 0.11$ & $17.03 \pm 0.06$ & 21.0 & nv & 1.35 & 32.8 & QSO \\
\hline $231557+005001$ & 348.986515 & 0.833474 & $1.945 \pm 0.095$ & $2.160 \pm 0.082$ & $2.012 \pm 0.089$ & $1.516 \pm 0.069$ & 0.35 & $<0.57$ & $3.41 \pm 0.07$ & 19.7 & & 2.52 & 32.5 & QSO \\
\hline $231713+000256$ & 349.305229 & 0.048771 & $13.192 \pm 0.181$ & $12.635 \pm 0.161$ & $11.598 \pm 0.160$ & $16.734 \pm 0.078$ & -0.28 & $31.86 \pm 0.11$ & $27.90 \pm 0.20$ & & & & & AGN \\
\hline $231846-000755$ & 349.690889 & -0.131884 & $4.550 \pm 0.086$ & $3.684 \pm 0.072$ & $3.976 \pm 0.080$ & $5.134 \pm 0.074$ & -0.33 & $4.18 \pm 0.11$ & $3.14 \pm 0.06$ & 19.5 & nv & 0.86 & 31.9 & QSO \\
\hline $232025+002744$ & 350.106120 & 0.462184 & $30.309 \pm 0.157$ & $25.698 \pm 0.134$ & $26.054 \pm 0.122$ & $35.256 \pm 0.095$ & -0.31 & $35.98 \pm 0.10$ & $21.68 \pm 0.27$ & & & 2.89 & 33.8 & QSO \\
\hline $232037+001335$ & 350.153115 & 0.226407 & $0.771 \pm 0.081$ & $0.957 \pm 0.062$ & $0.788 \pm 0.070$ & $0.542 \pm 0.066$ & 0.55 & $<0.51$ & $<0.39$ & & $\mathrm{nv}$ & $0.42^{\mathrm{a}}$ & 30.5 & AGN \\
\hline 232236-000712 & 350.650440 & -0.119883 & $11.177 \pm 0.163$ & $10.612 \pm 0.144$ & $10.984 \pm 0.125$ & $14.273 \pm 0.087$ & -0.29 & $24.53 \pm 0.10$ & $\ldots$ & 25.3 & $\ldots$ & $\ldots$ & $\ldots$ & AGN \\
\hline $232311-003122$ & 350.794080 & -0.522647 & $1.702 \pm 0.082$ & $1.786 \pm 0.077$ & $1.369 \pm 0.076$ & $1.289 \pm 0.066$ & 0.32 & $1.80 \pm 0.13$ & $\ldots$ & & & & $\ldots$ & AGN \\
\hline $232324+003328$ & 350.849824 & 0.557710 & $14.938 \pm 0.124$ & $13.590 \pm 0.099$ & $12.629 \pm 0.109$ & $9.361 \pm 0.063$ & 0.37 & $14.55 \pm 0.11$ & $\ldots$ & $\ldots$ & & & $\ldots$ & AGN \\
\hline $232656+000303$ & 351.732481 & 0.050827 & $2.510 \pm 0.131$ & $1.996 \pm 0.118$ & $2.536 \pm 0.119$ & $2.968 \pm 0.054$ & -0.39 & $0.93 \pm 0.10$ & $\ldots$ & 23.6 & $\ldots$ & $\ldots$ & $\ldots$ & AGN \\
\hline $232804+001904$ & 352.017137 & 0.317754 & $7.877 \pm 0.090$ & $6.876 \pm 0.079$ & $6.344 \pm 0.100$ & $4.273 \pm 0.067$ & 0.47 & $6.15 \pm 0.10$ & $\ldots$ & 21.6 & & & $\ldots$ & AGN \\
\hline 233210-003101 & 353.043262 & -0.516945 & $1.307 \pm 0.079$ & $1.573 \pm 0.075$ & $1.229 \pm 0.076$ & $1.102 \pm 0.064$ & 0.35 & $2.50 \pm 0.13$ & $\ldots$ & 20.3 & $\mathrm{nv}$ & 0.45 & 30.8 & AGN \\
\hline $233227-010443$ & 353.111298 & -1.078566 & $2.130 \pm 0.101$ & $2.225 \pm 0.093$ & $1.983 \pm 0.096$ & $1.543 \pm 0.075$ & 0.36 & $3.24 \pm 0.15$ & $\ldots$ & $\ldots$ & $\mathrm{nv}$ & 0.26 & 30.5 & AGN \\
\hline $233260-005129$ & 353.249596 & -0.857978 & $15.958 \pm 0.120$ & $14.168 \pm 0.102$ & $14.493 \pm 0.101$ & $21.498 \pm 0.098$ & -0.41 & $45.33 \pm 0.13$ & $\ldots$ & $\ldots$ & $\ldots$ & & $\ldots$ & AGN \\
\hline 233301-004501 & 353.255643 & -0.750179 & $2.952 \pm 0.134$ & $2.697 \pm 0.122$ & $3.097 \pm 0.132$ & $4.850 \pm 0.076$ & -0.57 & $6.75 \pm 0.14$ & $\ldots$ & $\ldots$ & & & $\ldots$ & AGN \\
\hline \multicolumn{15}{|c|}{ Timescale $<20 \mathrm{yr}$} \\
\hline $221650+005429$ & 334.210170 & 0.908094 & $1.673 \pm 0.067$ & $1.765 \pm 0.059$ & $1.598 \pm 0.064$ & $1.925 \pm 0.050$ & -0.09 & $<0.57$ & $<0.31$ & 21.4 & $\mathrm{nv}$ & $0.55^{\mathrm{a}}$ & 31.1 & AGN \\
\hline $221711+011038$ & 334.294275 & 1.177200 & $14.097 \pm 0.108$ & $15.139 \pm 0.081$ & $13.095 \pm 0.077$ & $18.508 \pm 0.057$ & -0.20 & $3.84 \pm 0.15$ & $7.51 \pm 0.07$ & 22.2 & $\ldots$ & $0.49^{\mathrm{a}}$ & 32.0 & AGN \\
\hline 221813-010344 & 334.554017 & -1.062315 & $8.724 \pm 0.082$ & $8.955 \pm 0.066$ & $7.971 \pm 0.072$ & $8.745 \pm 0.057$ & 0.02 & $<0.72$ & $0.79 \pm 0.10$ & $\ldots$ & $\ldots$ & $\ldots$ & $\ldots$ & AGN \\
\hline 223041-001644 & 337.672656 & -0.278969 & $3.805 \pm 0.084$ & $3.508 \pm 0.068$ & $3.763 \pm 0.070$ & $3.240 \pm 0.057$ & 0.08 & $<0.53$ & $0.49 \pm 0.06$ & $\ldots$ & $\ldots$ & & & AGN \\
\hline 223514-001425 & 338.806545 & -0.240294 & $3.858 \pm 0.161$ & $4.042 \pm 0.138$ & $4.265 \pm 0.169$ & $4.090 \pm 0.075$ & -0.01 & $10.45 \pm 0.11$ & $8.36 \pm 0.10$ & $\ldots$ & & 0.14 & 30.3 & AGN \\
\hline 230113-002941 & 345.306025 & -0.494588 & $9.942 \pm 0.094$ & $9.409 \pm 0.084$ & $9.257 \pm 0.092$ & $10.574 \pm 0.066$ & -0.12 & $2.54 \pm 0.13$ & $2.51 \pm 0.06$ & $\ldots$ & $\ldots$ & & & AGN \\
\hline $233002-002736$ & 352.507328 & -0.460065 & $5.492 \pm 0.157$ & $5.342 \pm 0.143$ & $5.742 \pm 0.147$ & $5.510 \pm 0.073$ & -0.03 & $<0.52$ & enter & 21.4 & $\ldots$ & 1.65 & 32.5 & QSO \\
\hline \multicolumn{15}{|c|}{ Serendipitous } \\
\hline 221515-005028 & 333.811588 & -0.841078 & $2.569 \pm 0.086$ & $1.989 \pm 0.062$ & $1.778 \pm 0.071$ & $1.787 \pm 0.058$ & 0.11 & $<0.68$ & $0.94 \pm 0.05$ & 21.4 & $\mathrm{nv}$ & $0.44^{\mathrm{a}}$ & 31.0 & AGN \\
\hline $223634-003352$ & 339.141345 & -0.564383 & $0.114 \pm 0.150$ & $0.897 \pm 0.123$ & $0.373 \pm 0.122$ & $0.394 \pm 0.069$ & 0.78 & $<0.66$ & $<0.63$ & 8.9 & & & $\ldots$ & RS CVn \\
\hline $230241+003450$ & 345.672648 & 0.580639 & $0.090 \pm 0.078$ & $0.422 \pm 0.069$ & $0.046 \pm 0.076$ & $0.127 \pm 0.065$ & 1.61 & $<0.51$ & $<0.28$ & 10.9 & & & $\ldots$ & $\mathrm{dKe}$ \\
\hline
\end{tabular}

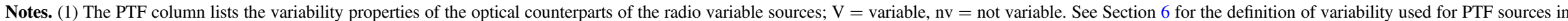

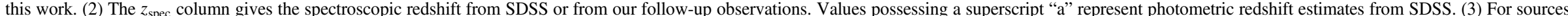

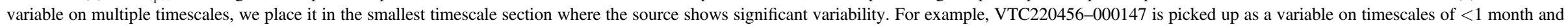

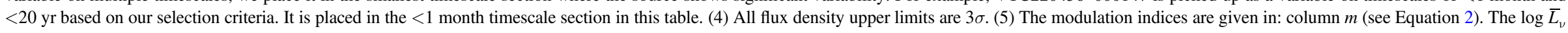
column gives the radio luminosity averaged over the four epochs of the pilot survey. 


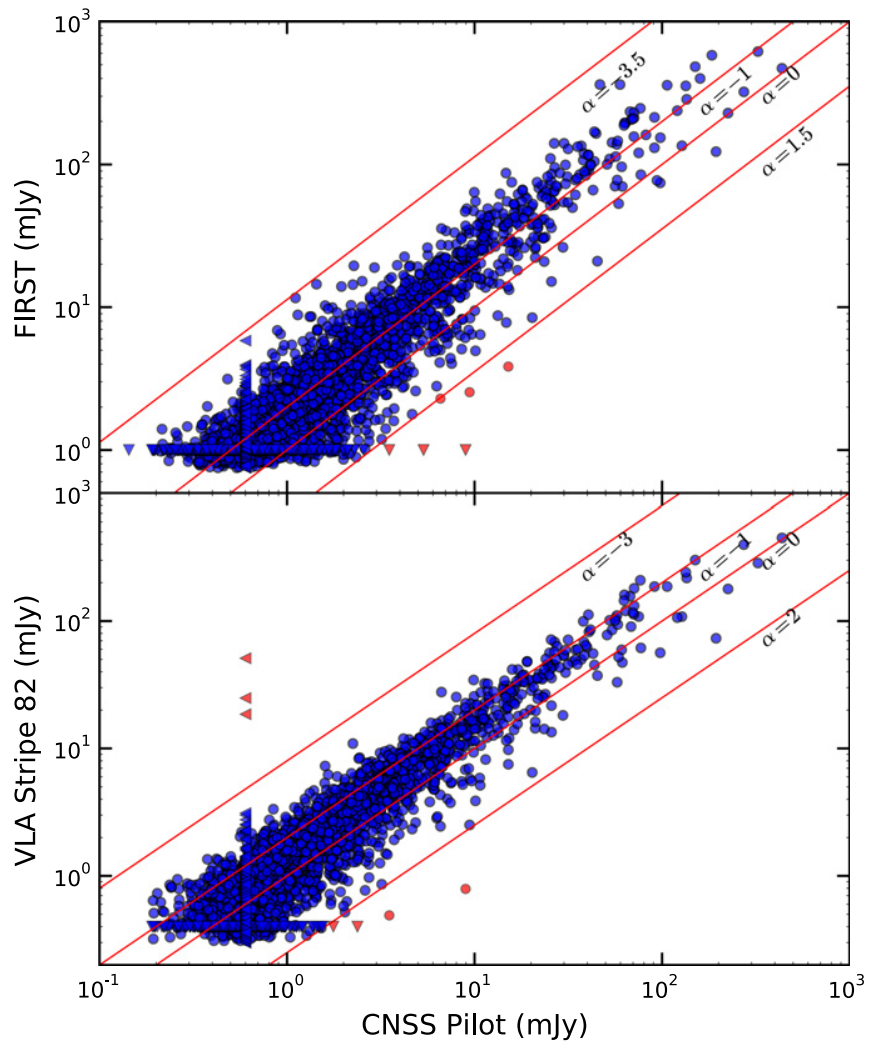

Figure 11. A comparison of the $3 \mathrm{GHz}$ peak flux densities of sources in the PSC with the $1.4 \mathrm{GHz}$ peak flux densities from the FIRST (top) and VLAStripe 82 (bottom) surveys. Triangles denote upper limits to the peak flux density. Red color denotes sources selected as variable candidates by our search criteria. See Section 5.2 for details.

and a summary of radio follow-up observations is given in Table 4.

\subsection{Transients On Timescales $<1$ Week}

\subsubsection{VTC225411-010651: AGN Flare}

This variable source has flux densities $0.644 \pm 0.063$, $0.782 \pm 0.050, \quad 0.300 \pm 0.056$, and $0.300 \pm 0.052 \mathrm{mJy}$ in epochs E1 to E4, and in the first two epochs it has spectral indices $-1.68 \pm 0.95$ and $0.39 \pm 0.68$, respectively, at $3 \mathrm{GHz}$. At the location of VTC225411-010651, the $1.4 \mathrm{GHz}$ peak pixel values from the FIRST and Hodge et al. (2011) surveys are $0.30 \pm 0.17$ and $0.12 \pm 0.10 \mathrm{mJy}$, respectively. To investigate further, we obtained follow-up observations of this object between 1 and $15 \mathrm{GHz}$ in two epochs (on 2012 September 02 and 17, 10 days and 25 days, respectively, after the epoch E3R2) with the VLA. The continuum radio spectra are shown in Figure 12. The first follow-up observation reveals a flat spectrum source in the $2-15 \mathrm{GHz}$ frequency range. The second observation indicates a spectrum peaked at about $1.5 \mathrm{GHz}$, with the optically thin part having a spectral index of $-0.99 \pm 0.16$. The spectrum is flat beyond $4 \mathrm{GHz}$. Submillimeter $(100 \mathrm{GHz})$ follow-up observations on 2012 September 09 with CARMA ${ }^{24}$ gave a nondetection with a $3 \sigma$ upper limit of $2.1 \mathrm{mJy}$.

There is no optical counterpart in PTF, but a faint SDSS DR7 (Abazajian et al. 2009) source having $r \simeq 23.3$

\footnotetext{
${ }^{24}$ All CARMA data were reduced with MIRIAD and VLA follow-up data with the NRAO CASA pipeline 1.2.0.
}

Table 4

Summary of the Radio Follow-up Observations of Variable and Transient Sources Reported in This Work

\begin{tabular}{|c|c|c|c|}
\hline Obs. Date & $\begin{array}{l}\text { Freq. } \\
(\mathrm{GHz})\end{array}$ & $\begin{array}{c}\mathrm{S} \\
(\mathrm{mJy})\end{array}$ & $\begin{array}{c}\sigma_{S} \\
(\mathrm{mJy})\end{array}$ \\
\hline \multicolumn{4}{|c|}{ VTC225411-010651 } \\
\hline 2012 Sep 01 & 2.4 & 0.504 & 0.039 \\
\hline 2012 Sep 01 & 3.2 & 0.604 & 0.028 \\
\hline 2012 Sep 01 & 3.8 & 0.569 & 0.033 \\
\hline 2012 Sep 01 & 4.5 & 0.596 & 0.024 \\
\hline 2012 Sep 01 & 5.1 & 0.623 & 0.023 \\
\hline 2012 Sep 01 & 7.1 & 0.641 & 0.021 \\
\hline 2012 Sep 01 & 7.7 & 0.646 & 0.021 \\
\hline 2012 Sep 01 & 13.2 & 0.584 & 0.035 \\
\hline 2012 Sep 01 & 13.8 & 0.672 & 0.032 \\
\hline 2012 Sep 01 & 14.2 & 0.639 & 0.035 \\
\hline 2012 Sep 17 & 1.2 & 1.130 & 0.079 \\
\hline 2012 Sep 17 & 1.8 & 1.363 & 0.077 \\
\hline 2012 Sep 17 & 2.4 & 1.109 & 0.051 \\
\hline 2012 Sep 17 & 3.1 & 0.841 & 0.040 \\
\hline 2012 Sep 17 & 3.8 & 0.671 & 0.068 \\
\hline 2012 Sep 17 & 4.5 & 0.596 & 0.049 \\
\hline 2012 Sep 17 & 5.1 & 0.623 & 0.047 \\
\hline 2012 Sep 17 & 7.1 & 0.641 & 0.044 \\
\hline 2012 Sep 17 & 7.7 & 0.646 & 0.045 \\
\hline \multicolumn{4}{|c|}{ VTC232939-004755 } \\
\hline 2012 Sep 01 & 2.4 & 0.739 & 0.065 \\
\hline 2012 Sep 01 & 3.0 & 0.701 & 0.048 \\
\hline 2012 Sep 01 & 3.4 & 0.740 & 0.054 \\
\hline 2012 Sep 01 & 4.8 & 0.778 & 0.027 \\
\hline 2012 Sep 01 & 7.4 & 0.741 & 0.021 \\
\hline 2012 Sep 01 & 13.5 & 0.598 & 0.026 \\
\hline 2012 Sep 01 & 14.5 & 0.568 & 0.027 \\
\hline
\end{tabular}

\begin{tabular}{|c|c|c|c|}
\hline \multicolumn{4}{|c|}{ VTC233002-002736 } \\
\hline 2012 Sep 01 & 2.4 & 6.846 & 0.095 \\
\hline 2012 Sep 01 & 3.2 & 9.294 & 0.071 \\
\hline 2012 Sep 01 & 3.8 & 9.641 & 0.108 \\
\hline 2012 Sep 01 & 4.5 & 10.519 & 0.073 \\
\hline 2012 Sep 01 & 5.1 & 10.558 & 0.071 \\
\hline 2012 Sep 01 & 7.1 & 9.612 & 0.070 \\
\hline 2012 Sep 01 & 7.7 & 9.381 & 0.073 \\
\hline 2012 Sep 01 & 13.2 & 7.269 & 0.102 \\
\hline 2012 Sep 01 & 13.8 & 6.836 & 0.097 \\
\hline 2012 Sep 01 & 14.2 & 6.487 & 0.103 \\
\hline 2012 Sep 01 & 14.8 & 6.217 & 0.098 \\
\hline 2012 Sep 17 & 1.2 & 1.829 & 0.189 \\
\hline 2012 Sep 17 & 1.8 & 3.791 & 0.179 \\
\hline 2012 Sep 17 & 2.4 & 7.437 & 0.082 \\
\hline 2012 Sep 17 & 3.1 & 9.327 & 0.067 \\
\hline 2012 Sep 17 & 3.8 & 10.380 & 0.123 \\
\hline 2014 May 29 & 1.3 & 2.396 & 0.179 \\
\hline 2014 May 29 & 1.9 & 4.253 & 0.240 \\
\hline 2014 May 29 & 2.4 & 7.095 & 0.188 \\
\hline 2014 May 29 & 3.0 & 7.511 & 0.109 \\
\hline 2014 May 29 & 3.4 & 7.272 & 0.094 \\
\hline 2014 May 29 & 8.4 & 4.984 & 0.051 \\
\hline 2014 May 29 & 9.4 & 4.562 & 0.053 \\
\hline 2014 May 29 & 10.4 & 3.955 & 0.055 \\
\hline 2014 May 29 & 11.4 & 3.474 & 0.081 \\
\hline 2014 May 29 & 13.2 & 3.295 & 0.074 \\
\hline 2014 May 29 & 13.8 & 3.505 & 0.070 \\
\hline 2014 May 29 & 15.7 & 2.937 & 0.076 \\
\hline 2014 May 29 & 16.3 & 2.949 & 0.076 \\
\hline
\end{tabular}


Table 4

(Continued)

\begin{tabular}{lccc}
\hline \hline Obs. Date & $\begin{array}{l}\text { Freq. } \\
(\mathrm{GHz})\end{array}$ & $\begin{array}{c}\mathrm{S} \\
(\mathrm{mJy})\end{array}$ & $\begin{array}{c}\sigma_{S} \\
(\mathrm{mJy})\end{array}$ \\
\hline & VTC221515-005028 & & \\
\hline 2014 May 29 & 1.3 & 2.759 & 0.137 \\
2014 May 29 & 1.9 & 2.549 & 0.175 \\
2014 May 29 & 2.4 & 2.654 & 0.080 \\
2014 May 29 & 3.0 & 2.373 & 0.069 \\
2014 May 29 & 3.4 & 1.935 & 0.067 \\
2014 May 29 & 4.5 & 2.155 & 0.061 \\
2014 May 29 & 5.1 & 2.058 & 0.055 \\
2014 May 29 & 7.1 & 1.337 & 0.047 \\
2014 May 29 & 7.7 & 1.198 & 0.048 \\
2014 May 29 & 13.2 & 0.487 & 0.059 \\
2014 May 29 & 13.8 & 0.710 & 0.054 \\
2014 May 29 & 15.7 & 0.554 & 0.060 \\
2014 May 29 & 16.3 & 0.464 & 0.059 \\
\hline
\end{tabular}

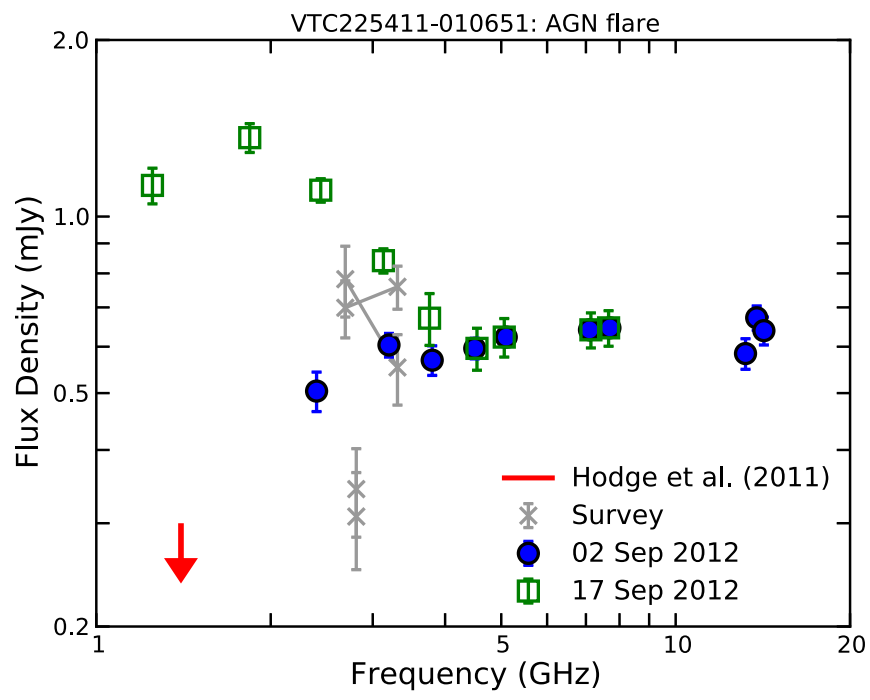

Figure 12. Radio continuum spectrum of VTC225411-010651 at two followup epochs (blue circles and green squares). The $3 \sigma$ upper limit at $1.4 \mathrm{GHz}$ from Hodge et al. (2011) is shown in red. The measurements from the four survey epochs are shown in gray. The first two epochs have spectral indices of $-1.68 \pm 0.95$ and $0.39 \pm 0.68$ within the $S$ band, respectively, and are indicated by gray crosses joined by straight lines between the two frequencies used for spectral index measurement. See Section 5.3.1 for details.

mag lies 1"! 8 away from the radio source position of VTC225411-010651. The photometric redshift from SDSS is $0.64 \pm 0.14$. Assuming a $1.4 \mathrm{GHz}$ quiescent flux density of $0.2 \mathrm{mJy}$, we can calculate the radio-to-optical flux density ratio for the host galaxy to be $\log \left(S_{1.4 \mathrm{GHz}} / S_{g}\right)=2.1$, which is typical of radio-loud AGNs (e.g., Padovani et al. 2011). WISE colors $(W 1-W 2=0.37 \pm 0.29 \mathrm{mag}$,

$W 2-W 3=3.90 \pm 0.51 \mathrm{mag}$ ) of the host galaxy are consistent with a LINER/(U)LIRG/spiral galaxy (Wright \& Eisenhardt 2010; Cutri et al. 2012).

The flat spectrum of this source in the first follow-up epoch indicates a jet with unresolved knots. Assuming a $3 \mathrm{GHz}$ quiescent flux density of $0.3 \mathrm{mJy}$, we obtain a radio luminosity of $(5.3 \pm 2.5) \times 10^{23} \mathrm{erg} \mathrm{s}^{-1} \mathrm{~Hz}^{-1}$ at the SDSS photometric redshift. It is thus likely that this source is a low-luminosity radio-loud $\mathrm{AGN}$, in which case the peaked spectrum in the
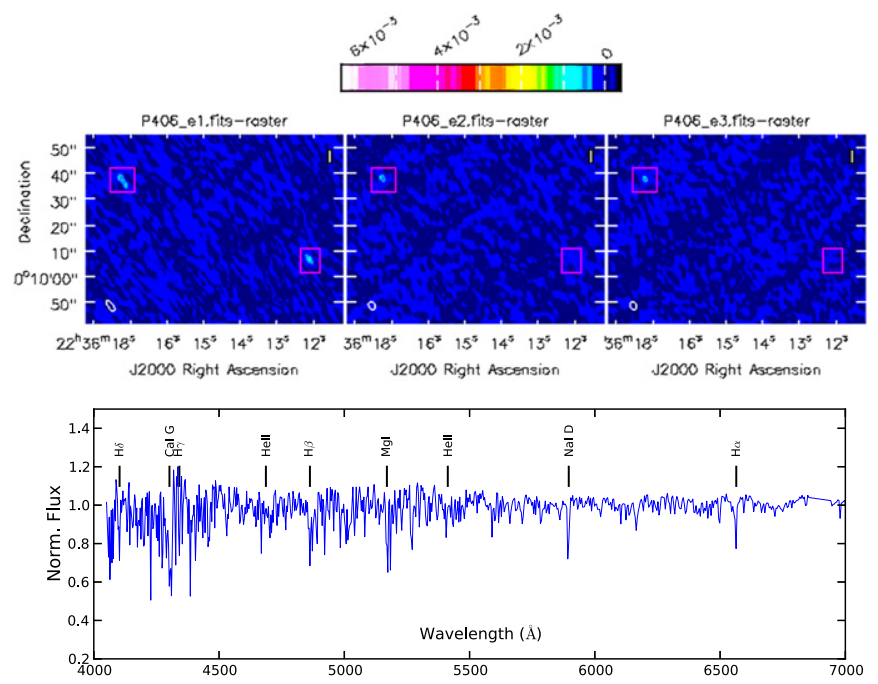

Figure 13. Top: the radio image cutouts from three epochs, E1, E2, and E3, showing VTC223612+001006 and a comparison $0.5 \mathrm{mJy}$ persistent source. The transient is detected with an $\mathrm{S} / \mathrm{N}$ of 9 in the first epoch and is below $\mathrm{S} / \mathrm{N}$ of 4 in the other two epochs. The color bar has units of Jy. Bottom: the continuum-normalized optical spectrum of VTC223612+001006 observed with ESI at Keck II on 2012 September 13. The spectrum has been binned with 20 data points in each bin. We classify this star as a late $\mathrm{G}$ to early $\mathrm{K}$ subgiant or giant. See Section 5.4.1 for details.

second follow-up epoch can be naturally explained as an intrinsic AGN flaring phenomenon. The flaring spectrum in Figure 12 is reminiscent of the shock-in-jet model that has been extensively used to explain flaring in bright quasars (Marscher \& Gear 1985; Türler et al. 2000; Fromm et al. 2011). The strong variability of VTC225411-010651 in the $S$ band between epochs E2 and E3 of our survey indicates that the flares in this AGN evolve on a timescale of less than 1 week. We note that current radio variability surveys are primarily focused toward the monitoring of blazars and bright quasars, where the flares at $\mathrm{GHz}$ frequencies evolve typically on timescales of a few months (e.g., Hovatta et al. 2008; Richards et al. 2011), and that VTC225411-010651 presents the first ever direct evidence of shocks propagating down the jet in submJy AGNs on a relatively short timescale.

\subsection{Transients On Timescales $<1$ Month}

\subsubsection{VTC223612+001006: RS CVn Flare}

VTC223612+001006 is detected as a transient on 2012 July 13 (epoch E1, regions R1). The peak pixel values at the location of the transient in epochs E1-E4 are $0.804 \pm 0.086$, $0.271 \pm 0.071, \quad 0.179 \pm 0.073, \quad$ and $\quad 0.098 \pm 0.057 \mathrm{mJy}$, respectively. The image cutouts near the source location for the three epochs are shown in Figure 13. A comparison source about 1.15 away from this transient candidate has relatively stable peak flux densities, 0.604, 0.584, 0.551, and $0.488 \mathrm{mJy}$ in epochs E1-E4, respectively, indicating that the variability of the candidate is secure. In the FIRST and Hodge et al. (2011) surveys, the peak pixel values at the location of the transient candidate are $0.13 \pm 0.11$ and $0.16 \pm 0.06 \mathrm{mJy}$, respectively.

The nearest optical counterpart of VTC223612 is the 10th $V$-band magnitude star HD $214129\left(\sim 1^{\prime \prime}\right.$ away), documented as a K0IV star by Torres et al. (2006) and as a G5/6III star by Kharchenko \& Roeser (2009). HD 214129 is a known visual binary and possibly a triple stellar system (e.g., Mason et al. 

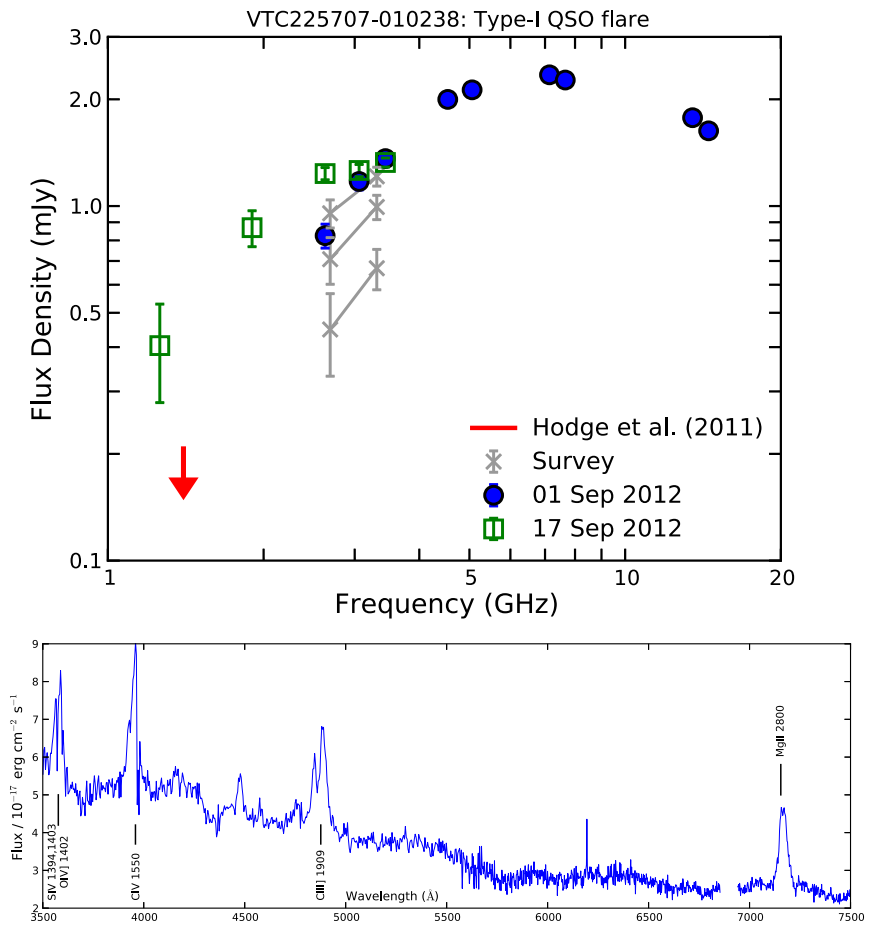

Figure 14. Top: the radio continuum spectrum of VTC225707-010238 at two follow-up epochs (blue circles and green squares). The $3 \sigma$ upper limit at 1.4 GHz from Hodge et al. (2011) is shown in red. The measurements from the first three survey epochs are shown in gray. See Section 5.4.2 for details. Bottom: the optical spectrum of VTC225707-010238 observed with LRIS at Keck I on 2012 August 18. The spectrum is typical of a blue quasar. We derive a redshift of 1.56

2001) and has been detected in the X-ray by ROSAT (1RXS J223612.5+001008; Voges et al. 1999). HD 214129 is saturated in PTF and SDSS, precluding the study of optical variability of this Galactic radio transient. We obtained an optical spectrum for this star using the Echelle spectrograph (ESI) at Keck II on 2012 September 13, which suggests a spectral type similar to K0IV. The spectrum does not show any strong emission lines within our spectral coverage between 4000 and $10000 \AA$. Figure 13 shows the binned optical spectrum between 4000 and $7000 \AA$. The narrow absorption line profiles and absence of Lorentzian wings indicate that the star is a subgiant or a giant. Fitting a blackbody to the photometric data from the SDSS, NOMAD, and WISE catalogs gives an effective temperature of $5000 \pm 200 \mathrm{~K}$.

If this were a main-sequence star, it would have a photometric parallax distance of $d \sim 65 \mathrm{pc}$, while if it is a giant, $d \sim 700$ pc. A subgiant can have any value between these extremes. An independent constraint on the distance comes from the space velocity. Using the proper motion and radial velocity, $\mu_{\alpha}, \mu_{\delta}=127.3,-39.4 \mathrm{mas} \mathrm{yr}^{-1}$ and $v_{r}=-3.3 \mathrm{~km} \mathrm{~s}^{-1}$ (Hog et al. 2000; Torres et al. 2006), we can calculate the space velocity (heliocentric) for $d \sim 65 \mathrm{pc}$ and $d \sim 700 \mathrm{pc}$ as $v \sim 40$ and $v \sim 440 \mathrm{~km} \mathrm{~s}^{-1}$, respectively (Johnson \& Soderblom 1987). Typically for stars we expect heliocentric space velocities between 0 and $100 \mathrm{~km} \mathrm{~s}^{-1}$ (e.g., Dehnen 1998). Therefore, we expect $40<d \lesssim 160$. Here we adopt a distance of $100 \mathrm{pc}$.

We used a $1 \mathrm{keV}$ collisionally excited plasma (APEC) model and an absorbing Galactic hydrogen column of $N_{\mathrm{H}}=5.05 \times$ $10^{20} \mathrm{~cm}^{-2}$ (derived from the $n h$ task in HEASoft) to convert the ROSAT/PSPC count rate from 1RXS $\left(0.23\right.$ counts $\left.\mathrm{s}^{-1}\right)$ in the
0.1-2.4 keV energy band to an unabsorbed flux of $3.2 \times 10^{-12} \mathrm{erg} \mathrm{cm}^{-2} \mathrm{~s}^{-1}$ using WebPIMMS. ${ }^{25}$ This corresponds to an X-ray luminosity of $3.8 \times 10^{30} \mathrm{erg} \mathrm{s}^{-1}$ at 100 pc. The X-ray luminosity, the X-ray-to-optical flux ratio of 1.4 $\times 10^{-3}$, and the spectral type all suggest that HD 214129 is an RS CVn-type binary. Accordingly, we expect the quiescent $3 \mathrm{GHz}$ radio luminosity of the transient to be $L_{R} \lesssim$ $10^{15 \pm 1} \mathrm{erg} \mathrm{s}^{-1} \mathrm{~Hz}^{-1}$ (Benz \& Güdel 1994; Güdel 2002; however, strictly speaking, their $L_{X}-L_{R}$ relationship is valid for $5 \mathrm{GHz}$ flux density). From epoch E4 we get the $3 \sigma$ upper limit on the quiescent $3 \mathrm{GHz}$ flux density of this transient as $2.4 \times 10^{15} \mathrm{erg} \mathrm{s}^{-1} \mathrm{~Hz}^{-1}$. Using a distance of $100 \mathrm{pc}$, we can estimate the flaring radio luminosity in epoch E1 as $1.1 \times 10^{16}$ erg $\mathrm{s}^{-1} \mathrm{~Hz}^{-1}$. From the binarity, the optical spectrum, radio, and X-ray luminosities, X-ray hardness ratios from ROSAT, and the X-ray-to-optical flux ratio, we conclude that this radio transient is a flare from an active binary system.

\subsubsection{VTC225707-010238: Flaring Type-I QSO}

VTC225707-010238 is a persistent but variable source with flux densities $0.602 \pm 0.073,1.218 \pm 0.069,0.914 \pm 0.065$, and $1.192 \pm 0.056 \mathrm{mJy}$ in the four epochs of our survey. The spectral indices within the $S$ band for the first three epochs are $1.93 \pm 1.42,1.16 \pm 0.53$, and $1.64 \pm 0.82$. The $1.4 \mathrm{GHz}$ peak flux densities from the FIRST and Hodge et al. (2011) surveys are $0.17 \pm 0.14$ and $0.17 \pm 0.07 \mathrm{mJy}$, respectively. We followed up this transient with the VLA on 2012 September 01 (2-15 GHz) and 2012 September 17 (1-4 GHz). The continuum radio spectra are shown in the top panel of Figure 14. Both follow-up spectra are peaked at a few GHz. On 2012 September 01, the peak is $2.5 \mathrm{mJy}$ at $7 \mathrm{GHz}$, while the 2012 September 17 spectrum is likely peaked at $3 \mathrm{GHz}$ with a flux density of $1.5 \mathrm{mJy}$. This might suggest that this object is a flaring gigahertz-peaked spectrum (GPS; young AGN) source. Submillimeter $(100 \mathrm{GHz})$ follow-up observations on 2012 September 09 with CARMA gave a nondetection with $3 \sigma$ supper limit of $2.1 \mathrm{mJy}$.

The optical counterpart of VTC225707-010238 is a blue point source in SDSS having $r=20.1 \mathrm{mag}$. The optical light curve from PTF shows intraday small-amplitude (submagnitude) variability. Follow-up spectroscopy with Keck I LRIS on 2012 August 18 (bottom panel of Figure 14) shows that this is a quasar at a redshift of 1.56. We therefore conclude that this transient is a flaring type-I quasar.

\subsection{Transients on Timescales $<1.5$ Year}

\subsubsection{VTC224730+000008: Flaring AGN}

VTC224730+000008 is a variable source with flux densities $284.2 \pm 1.2,272.9 \pm 0.8,260.3 \pm 1.0$, and $535.2 \pm 0.4 \mathrm{mJy}$ in the four epochs of our survey. Its $1.4 \mathrm{GHz}$ peak flux densities from the FIRST and VLA-Stripe 82 surveys are $317.1 \pm 0.1 \mathrm{mJy}$ and $388.7 \pm 0.1 \mathrm{mJy}$, respectively. The optical counterpart of VTC224730+000008 is a blue quasar, classified as a BL Lac-type object. Blazar-like optical variability is indeed evident in the PTF and SDSS photometric data. Optical spectra from the SDSS BOSS program indicate that the redshift is $z \sim 1$. Given this information, we conclude that VTC224730+000008 is an intermediate-redshift blazar.

\footnotetext{
${ }^{25}$ https://heasarc.gsfc.nasa.gov/cgi-bin/Tools/w3pimms/w3pimms.pl
} 

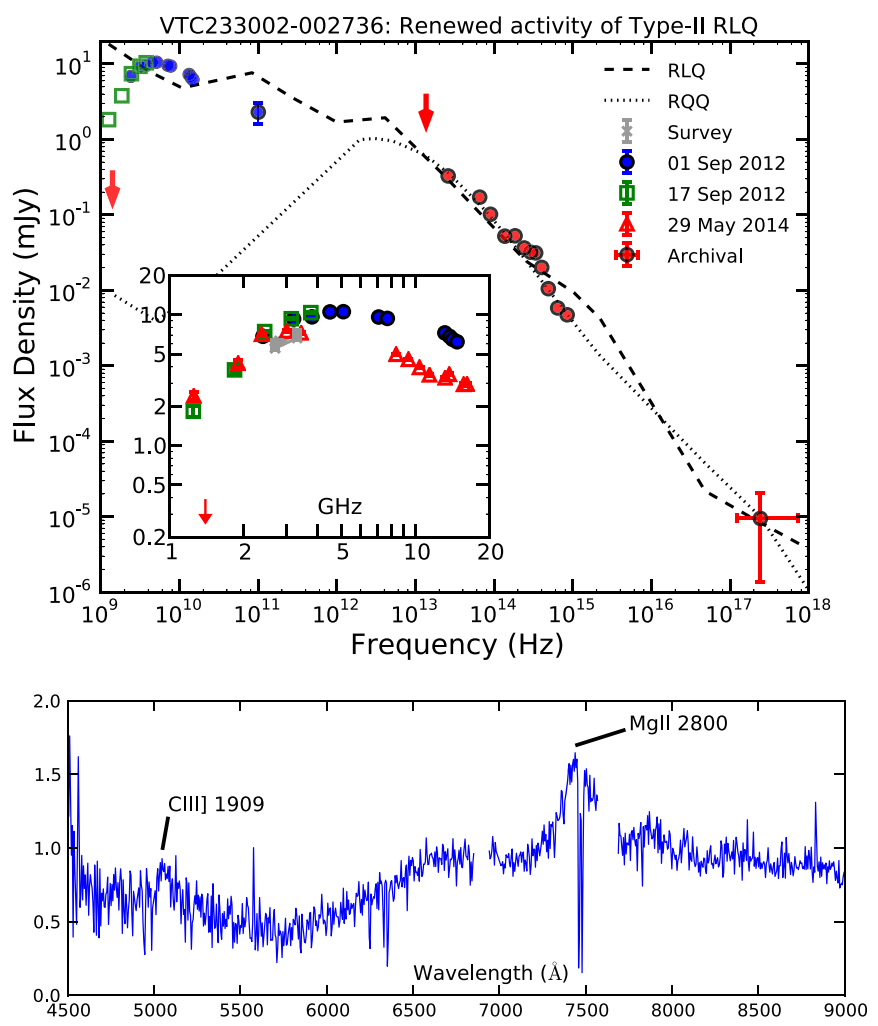

Figure 15. Top: the multi-frequency continuum spectrum of VTC233002-002736. Archival photometry is shown as filled red circles. The $3 \sigma$ upper limit at $1.4 \mathrm{GHz}$ from the FIRST survey and the upper limit from the WISE W4 filter are shown as red arrows. Blue circles and green squares indicate the first and second follow-up observations. Black dashed and dotted lines are average spectra for radio-loud and radio-quiet quasars from Elvis et al. (1994). The inset shows the zoom-in of the follow-up radio continuum spectra from the three epochs. Red triangles show data from the third follow-up epoch, and the measurements from the four survey epochs appear in gray. Bottom: the optical spectrum of VTC233002-002736 observed with DIEMOS at Keck II on 2012 September 14. The $y$-axis is flux in arbitrary units. Broad emission lines indicate a quasar at a redshift of 1.65. See Section 5.6.1 for details.

\subsection{Transients on Timescales $\lesssim 20$ Years}

\subsubsection{VTC233002-002736: Renewed Activity of Type-II QSO}

This is a transient with respect to the FIRST survey. Its flux density is $5.492 \pm 0.157,5.342 \pm 0.143,5.742 \pm 0.147$, and $5.510 \pm 0.073 \mathrm{mJy}$ in our four survey epochs, E1-E4, with spectral indices $1.42 \pm 0.23,0.86 \pm 0.22$, and $0.63 \pm 0.21$ in epochs E1-E3, within the $S$ band. The flux density in the FIRST survey at the location of VTC233002-002736 is $0.34 \pm 0.13$ mJy (mean epoch 1999.2). This implies an order of magnitude or more increase in flux density at $1.4 \mathrm{GHz}$ over the past decade and relatively stable flux density at $3 \mathrm{GHz}$ over the past 2 yr. We obtained follow-up observations with the VLA (1-15 GHz) on 2012 September 01, 17, and 2014 May 29. These observations reveal a GPS source with an optically thick spectral index of 2.1 between 1 and $3 \mathrm{GHz}$. On 2012 September 01, the spectrum peaks at $5 \mathrm{GHz}$ with a flux density of about $10 \mathrm{mJy}$, and the optically thin spectral index is -0.6 between 7 and $15 \mathrm{GHz}$. In the 2014 May 29 observations, spectral flattening is observed between 2 and $15 \mathrm{GHz}$ with respect to 2012 September 01 and the spectral peak appears to be at $3 \mathrm{GHz}$, suggesting a significant evolution in the spectrum beyond $3 \mathrm{GHz}$. The radio continuum spectra from the survey and follow-up observations are shown in the inset of the top panel of Figure 15. Follow-up observations from CARMA at $100 \mathrm{GHz}$ on 2012 September 09 give a detection at $2.3 \pm 0.7 \mathrm{mJy}$.

The SDSS counterpart of VTC233002-002736 is a reddish point source with $r=21.3 \mathrm{mag}$. The detection in the PTF is only marginal, and no meaningful optical variability information can be obtained. The SDSS light curve between 1998 and 2008 reveals submagnitude variability, typical of AGNs, on shortest timescales of a few weeks. VTC233002-002736 also has an X-ray counterpart in XMM-Newton (LaMassa et al. 2013). The multifrequency continuum spectrum of VTC233002-002736 is shown in the top panel of Figure 15. The optical to mid-infrared part of the spectrum agrees well with a type-II quasar template from Polletta et al. (2007). A comparison between the radio and optical flux densities $(R \simeq 2.4)$ indicates that this is a radio-loud quasar. We obtained a follow-up optical spectrum on 2012 September 14 with DIEMOS at Keck II (Figure 15, bottom panel). The spectrum shows broad $\mathrm{C}$ III] and $\mathrm{Mg}$ II spectral lines allowing us to calculate the redshift, $z=1.65$.

From the nature of the radio spectrum, the timescale of its evolution, and the nature of the host galaxy, we conclude that VTC233002-002736 is due to renewed jet activity from a typeII radio loud quasar, where the GPS spectrum is indicative of a young jet. The order-of-magnitude increase in flux density with respect to the FIRST survey could be indicative of an enhanced accretion phenomenon leading to an intensified jet. The flattening of spectral index at $\mathrm{GHz}$ frequencies between 2012 and 2014 may imply cessation in the increased accretion episode and/or the interaction of the jet with the circumnuclear material in the host galaxy.

\subsection{Transients Found Serendipitously}

This subsection describes two transients that were found while manually inspecting archival radio images and analyzing our $5 \sigma$ source catalogs. Being below the cutoff values that we have used, our variability criteria are not able to recover them. These two exemplars highlight the possibility that there are many more transients in our pilot survey data than have been reported in this work.

\subsubsection{VTC221515-005028: Extreme Variability of Flat- spectrum AGNs}

VTC221515-005028 has flux densities 2.569 \pm 0.086 , $1.989 \pm 0.062,1.778 \pm 0.071$, and $1.787 \pm 0.058 \mathrm{mJy}$ and spectral indices $0.24 \pm 0.30,0.03 \pm 0.31,0.68 \pm 0.38$, and $-0.45 \pm 0.72$ in epochs E1-E4 of our survey. It is absent in the FIRST survey (mean epoch 1996.1), where the flux density at that location is $0.25 \pm 0.10 \mathrm{mJy}$, but present in the $1.4 \mathrm{GHz}$ Stripe 82 survey catalog (Hodge et al. 2011) with a peak flux density of $0.79 \mathrm{mJy}$. For the transient search on timescales $\lesssim 20 \mathrm{yr}$ (Section 5.6) we assumed the upper limit to the flux density in FIRST as $1 \mathrm{mJy}$ and hence did not recover this source as a transient using our spectral index criterion. We carried out radio follow-up observations with the VLA (1-15 GHz) on 2014 May 29. The radio continuum spectrum is shown in Figure 16. The source appears to have a flat spectrum between 1 and $5 \mathrm{GHz}$ and a spectral index of -1.1 between 5 and $15 \mathrm{GHz}$.

The optical counterpart of VTC221515-005028 is a faint red galaxy having $r \simeq 20.8$ mag. From the PTF light curve it is 


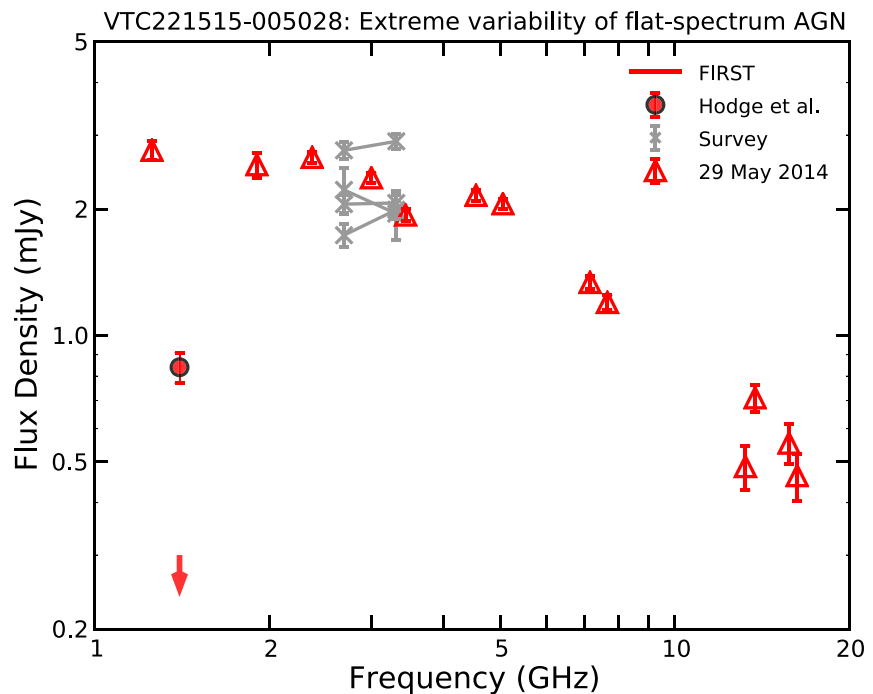

Figure 16. The radio continuum spectrum of VTC221515-005028 at the follow-up epoch 2014 May 29 (red triangles). The $3 \sigma$ upper limit from the FIRST survey is shown with the red arrow, and the detection from the Hodge et al. (2011) survey is marked by a red circle. The measurements from the four survey epochs are shown in gray. See Section 5.7.1 for details.

seen to exhibit stochastic variability with a maximum amplitude of one magnitude in the $R$ band, and having a shortest timescale of about 3 days. The SDSS light curve between 1999 and 2008 reveals submagnitude variability and intermittent flares with amplitudes up to $r=2$ mag on a $\sim 1$ day timescale. The photometric redshift from SDSS is 0.37. The radio-to-optical flux density ratio for the host galaxy, $R=\log \left(S_{1.4 \mathrm{GHz}} / S_{g}\right) \simeq 2.8$, and the radio luminosity at $1.4 \mathrm{GHz}, 9.0 \times 10^{29} \mathrm{erg} \mathrm{s}^{-1} \mathrm{~Hz}^{-1}$, suggest a low-luminosity radio-loud AGN. We thus conclude that VTC221515-005028 is an extremely variable flat-spectrum AGN.

\subsubsection{VTC230241+003450: Flare from dKe Star}

VTC230241+003450 is detected as a $6 \sigma$ source on 2012 August 15 (epoch E2, regions R2) and is not detected in the other epochs. The peak pixel values at the location of this transient in epochs E1-E4 are $0.090 \pm 0.078,0.422 \pm 0.069$, $0.046 \pm 0.076$, and $0.127 \pm 0.065 \mathrm{mJy}$, respectively. In the FIRST and Hodge et al. (2011) surveys, the peak pixel values at the location of the transient candidate are $0.13 \pm 0.11$ and $0.16 \pm 0.06 \mathrm{mJy}$, respectively. The optical counterpart of this transient is an 11th magnitude star, SDSS J230241.41 +003450.2 , classified as a K4Ve star by Torres et al. (2006). There is no evidence for binarity. Fitting a blackbody to the photometric data from SDSS, the NOMAD catalog, and WISE gives an estimate of the effective temperature, $3800 \pm 500 \mathrm{~K}$, and the distance, $70 \pm 20 \mathrm{pc}$ (assuming the radius of a main-sequence star). For SDSS J2302+00 we derive a $v \sin (i)$ of $85 \mathrm{~km} \mathrm{~s}^{-1}$. This star has a ROSAT counterpart, 1RXS $\mathrm{J} 230240.3+003453,17^{\prime \prime} \pm 12^{\prime \prime}$ away, whose hardness ratios are consistent with those of a coronal emitter. We used a $1 \mathrm{keV}$ APEC model in WebPIMMS to convert the ROSAT/PSPC count rate to a flux of $1.1 \times 10^{-12} \mathrm{erg} \mathrm{cm}^{-2} \mathrm{~s}^{-1}$ in the $0.1-2.4 \mathrm{keV}$ energy band. The $\mathrm{X}$-ray-to-optical flux ratio is 0.002 , and the X-ray luminosity at a distance of $70 \mathrm{pc}$ is $6.4 \times 10^{29} \mathrm{erg} \mathrm{s}^{-1}$, both values being consistent with a $\mathrm{dKe}$ star. The quiescent $3 \mathrm{GHz}$ radio luminosity of VTC230241+003450 can therefore be estimated as

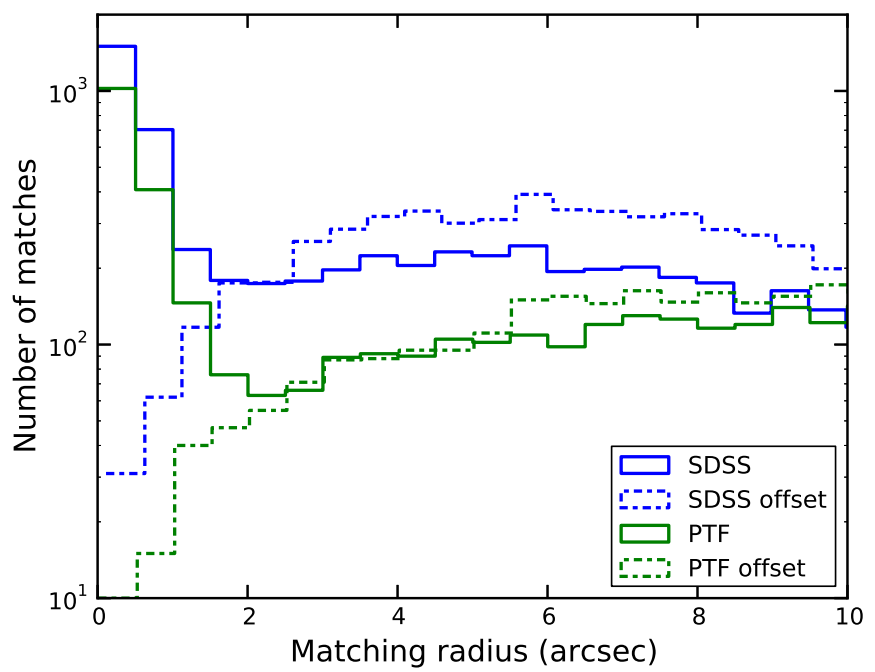

Figure 17. The number of optical matches for the radio sources in E2CAT for different matching radii. Solid lines indicate the matching process carried out using the actual radio source positions, and the dot-dashed lines indicate background matches found by offsetting the radio source positions by $1^{\prime}$ in an arbitrary direction. Blue and green lines show radio vs. SDSS and PTF matching, respectively.

$6.4 \times 10^{14 \pm 1} \mathrm{erg} \mathrm{s}^{-1} \mathrm{~Hz}^{-1}$ (Benz \& Güdel 1994; Güdel 2002). This can be compared with the flaring luminosity, $5.9 \times 10^{15} \mathrm{erg} \mathrm{s}^{-1} \mathrm{~Hz}^{-1}$. We conclude that this transient is a flare from a dKe star.

\section{OPTICAL PROPERTIES OF RADIO SOURCES}

PTF carried out a concurrent optical survey that resulted in the identification of a few hundred thousand sources per epoch down to a limiting magnitude of $R \simeq 21 \mathrm{mag}^{26}$ Our radio survey further benefits from the presence of SDSS deep co-add images containing more than 1 million objects over the $50 \mathrm{deg}^{2}$ to $r \simeq 23.5 \mathrm{mag}$ (Annis et al. 2014). To find optical counterparts of radio sources, we followed the procedure from Hodge et al. (2011). We matched each source in the E2CAT with the SDSS and PTF catalogs using a 15" matching radius and selected only the nearest match. To understand the false matching rate, we repeated the search by offsetting the radio source positions by $1^{\prime}$ in an arbitrary direction. The resulting number of matches as a function of the matching radius is plotted as a histogram in Figure 17. Based on these results, we choose a matching radius of $1^{\prime \prime}$ for SDSS and 1". 5 for PTF to achieve a false matching rate of less than $3 \%$ and completeness better than $85 \%$. The PTF source positions for the faintest objects are known to have a larger scatter than theoretically expected, and hence the larger matching radius of 1.15 is reasonable. Using these matching radii, we found the optical counterparts of sources in the PSC. The corresponding matching fraction in SDSS as a function of the $r$-band magnitude is shown graphically in Figure 18. Forty-nine percent of the radio sources in the PSC have an optical counterpart down to the SDSS $r$-band limit. ${ }^{27}$ Hodge et al. (2011), using the deep co-added SDSS images (limit $i$

\footnotetext{
26 Note that, for the $60 \mathrm{~s}$ snapshots taken by PTF, the CCDs are saturated at $\sim 10-11$ mag. This represents the lower limiting magnitude of the optical study carried out here.

27 If all the radio sources (components) from the E2CAT are matched with SDSS sources, then the completeness is much lower, $35 \%$.
} 


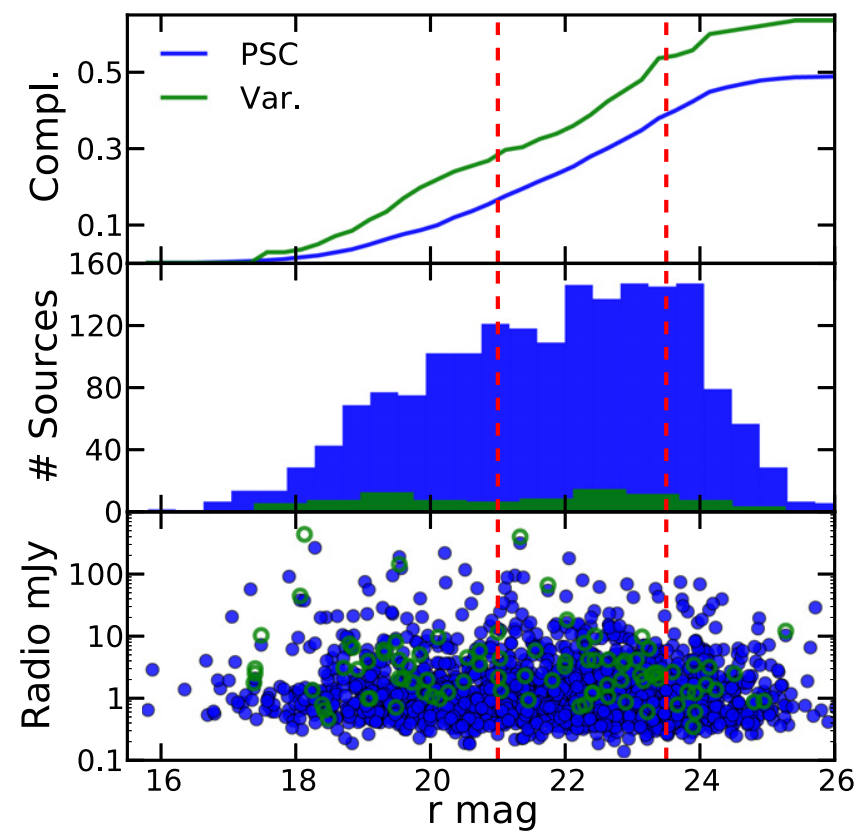

Figure 18. Optical counterparts of persistent (blue) and variable (green) radio sources in the PSC. The $x$-axis is $r$-band magnitude from SDSS. The top panel gives the completeness of the radio-optical cross-matching, the middle panel shows histograms of persistent and variable radio sources with optical counterparts, and the bottom panel plots the radio flux densities vs. optical magnitudes. The approximate limiting magnitudes for PTF (per epoch; $R=21 \mathrm{mag}$ ) and SDSS (Stripe 82 co-add; $r=23.5 \mathrm{mag}$ ) are shown as red dashed lines.

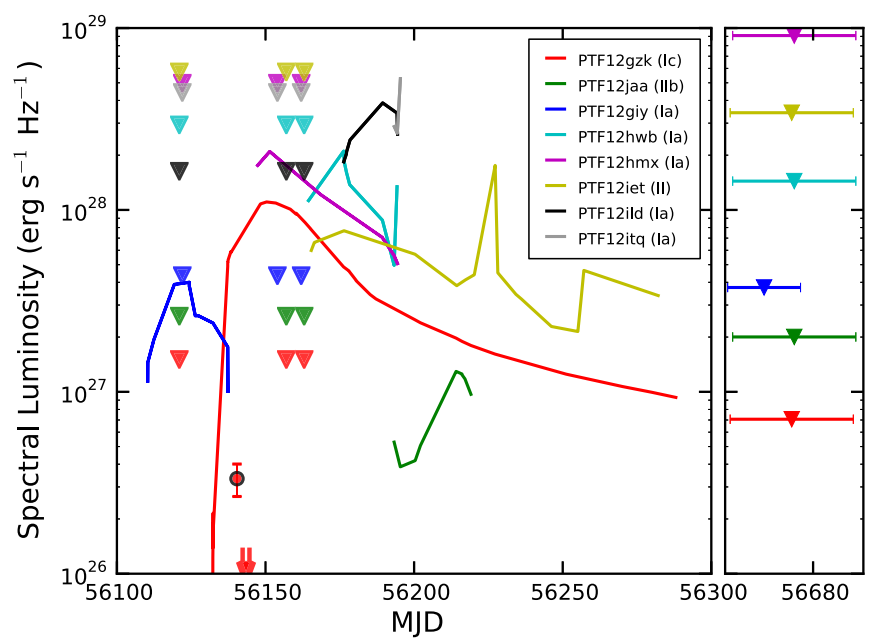

Figure 19. Light curves of the eight spectroscopically confirmed supernovae found in the PTF survey. Each supernova is represented by a unique color. Optical photometric data from PTF and follow-up observations are shown as solid lines. The $3 \sigma$ radio upper limits from the Jansky VLA survey (four epochs) are shown as downward-pointing triangles. For PTF12gzk, the radio detection (red circle and error bar) and $3 \sigma$ upper limits (downward-pointing red arrows) at $5 \mathrm{GHz}$ from Horesh et al. (2013) are also marked. See Section 6 for details.

$\sim 23.5 \mathrm{mag}$ ), found a matching ratio (within a $1^{\prime \prime}$ radius) of $44.4 \%$. For radio variable sources, we found a somewhat higher matching fraction of $63 \%$ (Figure 18), which is also in agreement with Hodge et al. (2011).

The modest optical matching rate in our medium-wide, medium-deep survey was not unexpected. Ivezic et al. (2002)

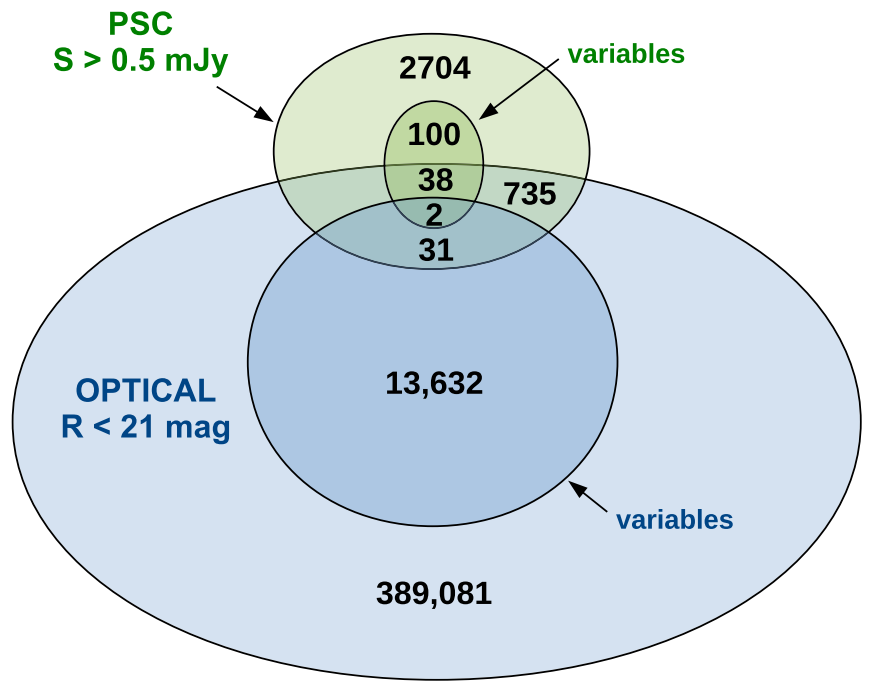

Figure 20. Venn diagram showing the number of persistent and variable optical sources from PTF and radio sources from the PSC. The sets plotted here are not to scale.

compared the SDSS (limit $r \sim 22 \mathrm{mag}$ ) and the FIRST (5 $\sigma \sim 1$ mJy) surveys in $1230 \mathrm{deg}^{2}$ of the sky and matched about $30 \%$ of the 108,000 FIRST radio sources. McMahon et al. (2002) looked at the 382,892 FIRST sources in the north Galactic cap and looked for optical matches using the APM scans of POSS-I plates $(R \sim 20 \mathrm{mag}, B \sim 21.5 \mathrm{mag}$ ) for an $18 \%$ identification rate $(70,000$ sources). The optical match rate improves substantially in deep, narrow surveys. For example, Huynh et al. (2008) use Australia Telescope Compact Array (ATCA) data taken toward the Hubble Deep Field (HDF) at 20, 11, 6, and $3.6 \mathrm{~cm}$, establishing a $66 \%$ matching of optical counterparts to $I=23.5 \mathrm{mag}$. There is a strong color dependence, with the matching rate increasing from the blue to the near-infrared (El Bouchefry \& Cress 2007; Smolcic et al. 2008). Match rates approach $100 \%$ with the use of deep infrared data (Bonzini et al. 2012).

The near-real-time optical transient search carried out via image subtraction during the 3 month high-cadence PTF observations resulted in approximately 8 million detections. Following standard practice, about 0.9 million of these (corresponding to only $\sim 50,000$ unique optical sources) were identified in machine learning software as unlikely to be image subtraction artifacts, and further filtering was carried out using several stringent selection criteria (at least two detections within $1 \mathrm{hr}$, no coincidence with stellar counterparts or AGNs, etc.) and automated classifiers (Bloom et al. 2012). This was followed by human inspection of the subtracted images, light curves, and automated classifications, and the list was narrowed down to 193 candidates for further follow-up. Out of the 193 candidates, only 10 sources were ultimately followed up spectroscopically, among which are 8 confirmed supernovae, PTF12gzk (SN Ic, $z=0.014$ ), 12jaa (IIb, 0.024), 12giy (Ia, 0.029), 12hwb (Ia, 0.056), 12hmx (Ia, 0.085), 12iet (II, 0.095), 12ild (Ia, 0.17), and 12itq (Ia, 0.22). The multiwavelength observations of PTF12gzk have been discussed at length by Ben-Ami et al. (2012) and Horesh et al. (2013). In Figure 19 we plot the optical light curves of these eight supernovae, as well as the upper limits to their spectral luminosity in the radio. The radio nondetections are in accordance with the expected flux densities of Type II, Ic, and Ia supernovae (Table 1). The 
radio detection and subsequent nondetections of PTF12gzk from Horesh et al. (2013) are also shown for reference, and these data emphasize the need for deep radio observations for optically bright supernovae such as the ones generally found by PTF. After all the survey observations were complete, we compiled optical light curves of sources using the catalogs hosted at IPAC (Section 4). In order to enable robust variability search, we selected only those optical sources having more than 16 reliable observations (unflagged in SExtractor) and $R$-band magnitudes between 10 and 23. There are 402,747 such sources in the PTF database, with the $90 \%$ completeness of this sample corresponding to approximately an $R$-band limiting magnitude of $21.802(0.2 \%)$ of these sources have counterparts in the PSC (i.e., 22\% of the PSC sources have optical counterparts). A total of $13,667(3.4 \%)$ are optical variables. ${ }^{28}$ Only 42 radio sources have optical variable counterparts among the sample of 3652, while only two of these are variable also in the radio. Thus, given the limiting magnitude of $R=21$ mag in optical and our source detection threshold of $\sim 0.5 \mathrm{mJy}$ in the radio, we find that the overlap between optical variables and radio variable sources is extremely small. These demographics are succinctly presented in a Venn diagram in Figure 20.

Here we are less concerned about optical-only transients and will focus instead on the optical variability properties of the radio transients and variables. A study of optical-only variables and transients from synoptic surveys is better done elsewhere in the literature (e.g., Drake et al. 2009; Rau et al. 2009). We have obtained photometric data from PTF and SDSS after searching for counterparts as described above. A compilation of the optical light curves of the radio variable sources is given in Figure 21. A majority of the PTF light curves reveal submagnitude variability, while the SDSS light curves show gradual submagnitude or magnitude-level variability on timescales of years. This is characteristic of AGNs, where fluctuations in the accretion rate or other causes give rise to optical variability on a wide range of timescales. Coupled with radio variability information, it seems likely that all of these sources are AGNs, excepting the ones associated with known stars.

\section{SUMMARY AND DISCUSSION}

We have carried out a dedicated radio transient survey in a $50 \mathrm{deg}^{2}$ region of the SDSS Stripe 82. This survey is a pilot for the CNSS, a multi-epoch survey of the entire $\sim 270 \mathrm{deg}^{2}$ of Stripe 82 , which is currently under way. The pilot observations were carried out with the Jansky VLA between 2 and $4 \mathrm{GHz}$ over four epochs spaced logarithmically in time, i.e., having cadences of 1 week, 1 month, and $1.5 \mathrm{yr}$. The median rms noise per epoch is between 50 and $90 \mu \mathrm{Jy}$. With this pilot we have successfully demonstrated the near-real-time calibration, imaging, and transient search capability with the Jansky VLA data. We have found 142 sources displaying fractional variability beyond $30 \%$. Based on radio follow-up observations, radio and optical variability, radio spectral indices, and multiwavelength archival photometric data, almost all of these are likely to be due to shocks in AGN jets. We have also found two bona fide radio transients associated with Galactic objects, a flare from an RS CVn binary and a dKe flare. Comparison of our pilot survey

\footnotetext{
${ }^{28}$ Given the optical light curves, sources having a $\chi^{2}$ probability less than 1 in 500,000 and variability $>30 \%$, i.e., a standard deviation of $>0.28$ mag, were selected as optical variables. These criteria were designed to be similar to the radio variability criteria.
}

data with the FIRST and VLA-Stripe 82 surveys has (unexpectedly) revealed a few additional, highly variable and transient sources on timescales of 5-20 yr. These sources, most of which either are previously known radio sources or have optical AGN hosts, are likely associated with renewed AGN activity. Two other transients, found serendipitously, suggest that there are indeed many more transients in this data set than the ones that we have found through our stringent selection criteria. We encourage the astronomical community to take advantage of the data from this pilot radio survey (see Section 3.2.5 for the data release website) for finding these hidden transients and for other science.

A high-cadence optical survey using PTF was carried out over 3 months, contemporaneous with the first three epochs of the radio survey. The motivation behind this joint survey was the selection of radio transients for rapid follow-up, preliminary classification of radio variables and transients based on optical light curves, and a direct comparison of the dynamic radio and optical skies. As noted by previous radio and optical studies (see Section 6), 20\% of the persistent radio sources have an optical counterpart above the PTF limiting magnitude of 21 , while $50 \%$ have counterparts above the SDSS limiting magnitude of 23.5 in the red filter. We have found primarily slowly evolving radio transients and, within the sensitivity limits of our PTF and pilot Jansky VLA surveys, very little overlap between the optical and radio variable skies. Only $0.05 \%$ of the radio point sources and $<0.001 \%$ of the optical sources are variable at both frequencies. The fraction of strong variables per square degree of the radio decimetric sky is at least an order of magnitude smaller than those in the optical sky. We will now use the radio data to assess the degree of variability and the transient rate of the radio sky, and make recommendations for future surveys.

\subsection{Comparison of Variability with Previous Surveys}

We found only a small fraction $\left(142 / 3652=3.9_{-0.9}^{+0.5} \%\right)$ of the point sources varying by $>30 \%$ on week-month-year timescales. A majority of these are variable only on a 1.5 year timescale, and as described in Section 5.2, this variability fraction is to be taken as the upper limit. Several previous studies at $1.4 \mathrm{GHz}$ (Frail et al. 1994; de Vries et al. 2004; Croft et al. 2010; Bannister et al. 2011a, 2011b; Thyagarajan et al. 2011; Mooley et al. 2013) have shown that the fraction of variables on timescales between minutes and years, and flux densities between $0.1 \mathrm{mJy}$ and $100 \mathrm{mJy}$, is $\sim 1 \%$ or less. Similar to these studies, ${ }^{29}$ we see that the $3 \mathrm{GHz}$ sky is not highly variable on timescales longer than a few days. Radio follow-up observations, optical and radio light curves, and multiwavelength archival data of the radio variable sources suggest that almost all of these are shock-related flaring in the jets of AGNs.

Our choice of modulation index for measuring variability and selection threshold introduces a bias toward selecting sources with large flux densities. A source in the PSC would have to have a mean flux density of $1.4 \mathrm{mJy}$ in order to detect a $30 \%$ fractional variability, while at the $7 \sigma$ source detection limit of $\sim 0.5 \mathrm{mJy}$, a source would have to have $|m|>3 / 4$ $\left(f_{\text {var }}>120 \%\right)$ in order to be identified as a significant variable. Fifteen strong variables (i.e., sources having $|m|>2 / 3$, or

\footnotetext{
29 See http://tauceti.caltech.edu/kunal/radio-transient-surveys/index.html for a description of past variability studies.
} 

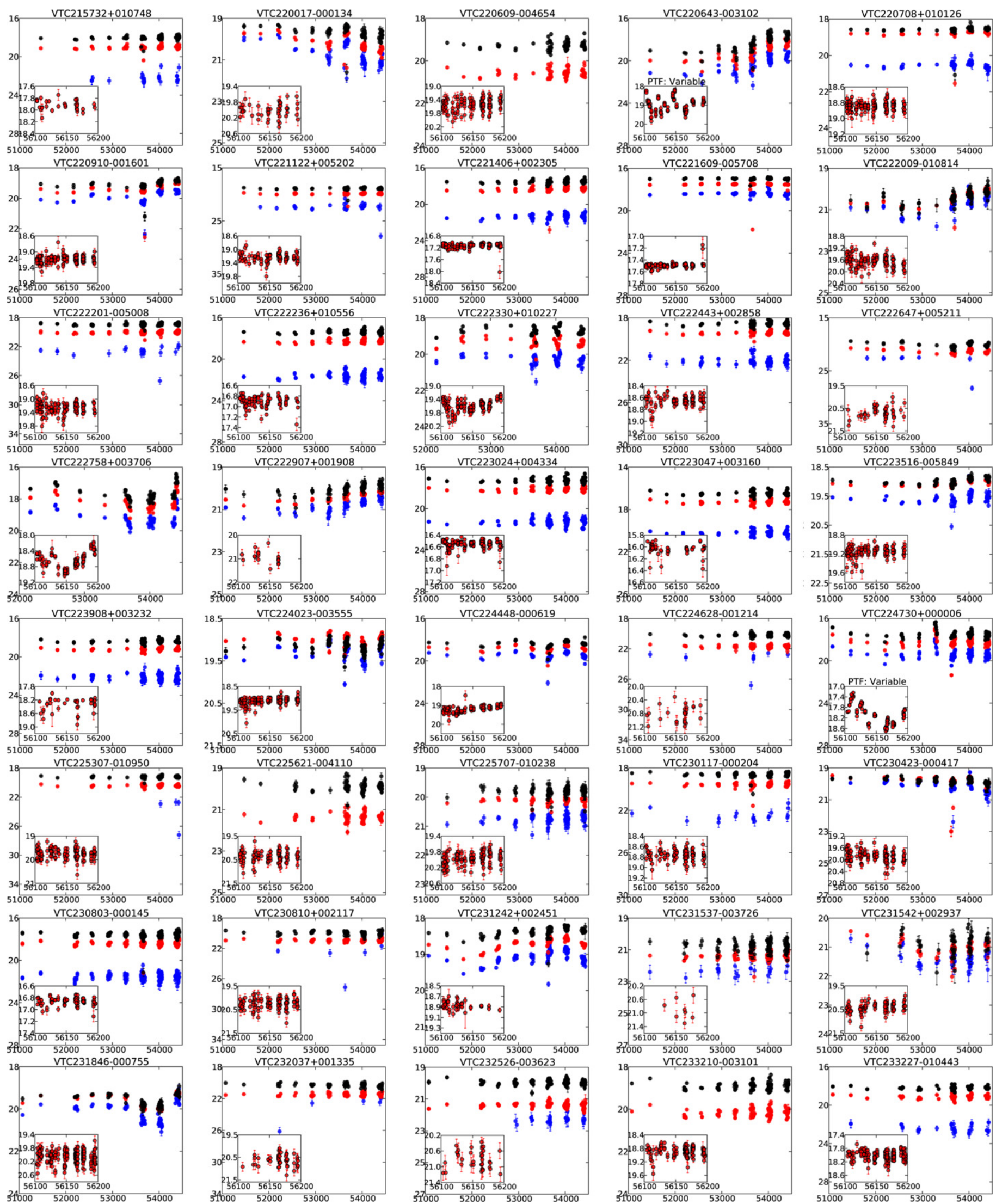

Figure 21. Well-sampled optical light curves of radio variable sources from SDSS (showing variability on timescales of yr) and PTF (inset; showing variability on timescales of weeks). A majority of these light curves reveal submagnitude or magnitude-level variability on timescales of months to yr, indicative of AGNs. For SDSS, the $u$-, $r$-, and $z$-band light curves are shown as blue, red, and black circles with error bars, respectively. For PTF, the $R$-band light curve is shown. The title of the inset describes whether the source is classified as a variable in PTF based on our variability criteria. See Section 6 for more details. The $y$-axis in each plot is the magnitude, and the $x$-axis is the Modified Julian Date (MJD). The $x$-axis runs from 51,000 to 54,500 in all the panels and from 56,100 and 56,200 in all insets. Note that the MJDs 51,000, 54,000, 56,100, and 56,200 correspond to 1998 July, 2006 September, 2012 June, and 2012 October, respectively. 
$f_{\text {var }}>100 \%$ ) were identified in our survey, but only 3000 sources in the PSC are bright enough ( $>0.55 \mathrm{mJy})$ to have been identified as a strong variable. We can therefore conclude that the fraction of strong variables is less than $1 \%$.

Radio variability appears to be a function of timescale; the variability on hour-day timescales contrasts with the variability on week-year timescales. Ofek et al. (2011) found that $\sim 30 \%$ of point sources brighter than $1.5 \mathrm{mJy}$ at $5 \mathrm{GHz}$ were variable, with the majority of the sources varying on timescales $<10$ days. This variability is primarily small amplitude (modulation indices of less than 0.2). The structure function of the variable sources constructed by Ofek et al. (2011) shows a sharp rise on timescales of $<1$ day, a shallow but steady rise between 1 and 10 days, and a roughly constant value beyond 10 days. A large fraction of variables displaying small variability amplitudes on short timescales were also found by the MASIV survey (Lovell et al. 2008) conducted at $5 \mathrm{GHz}$. The findings of Ofek et al. (2011) and Lovell et al. (2008) suggest that the low-amplitude variability on timescales of $<10$ days is mostly extrnisic. Among the Ofek et al. (2011) radio sources, only $0.3 \%$ have modulation indices greater than 0.2 on timescales less than $2 \mathrm{yr}$. The variability fraction that we find on week-month timescales in our pilot survey is similar $(\lesssim 1 \%)$ and agrees with the variability fraction found in narrow-deep surveys by Frail et al. (1994), Carilli et al. (2003), and Mooley et al. (2013), wide-field surveys such as Williams et al. (2013), and other studies between 1 and $5 \mathrm{GHz}$ on week-month-year timescales. On a 1.5 year timescale we find the variability fraction to be less than $4 \%$. Recently, Hodge et al. (2013) compared the $1.4 \mathrm{GHz}$ VLA-Stripe 82 and FIRST surveys and found $\sim 12 \%(6 \%$ per epoch $\times 2$ epochs) of the sources having fractional variablility larger than 0.3 on timescales between 7 and $22 \mathrm{yr}$, the majority of which were AGNs. While it is possible that, on these longer timescales, the fraction of variable sources in the radio sky is large, some of the Hodge et al. (2013) variables could be artificial and solely due to angular resolution differences between the VLA-Stripe 82 and FIRST surveys. ${ }^{30}$ Indeed, Becker et al. (2010), Bannister et al. (2011b), and Croft et al. (2010) find that the fraction of variables on similar timescales is a few percent or less.

Radio variability (especially extrinsic) is expected to have a frequency dependence, but this aspect of variability has not been extensively studied. We can only use the results of past blind surveys to conclude that the fraction of strong variables is less than a few percent between frequencies of 1 and $5 \mathrm{GHz}$, between flux densities of $\sim 0.3$ and $\sim 100 \mathrm{mJy}$, and over a wide gamut of timescales (between 1 day and several years).

In our pilot survey, we found a single AGN, VTC233002-002736, with flux density $>3 \mathrm{mJy}$ at $1.4 \mathrm{GHz}$, that appears to have increased in flux density by more than a factor of 10 over the past $15 \mathrm{yr}$. Similar objects may have been found earlier, by Bannister et al. (2011a, 2011b), in the SUMSS survey (e.g., J201524-395949 or J060938-333508). It is likely that such phenomena are a result of episodes of enhanced accretion leading to increased jet activity (see KunertBajraszewska et al. 2006, 2010; Czerny et al. 2009; Elitzur

\footnotetext{
${ }^{30}$ However, Hodge et al. (2013) assert that the fractional variability of 0.30 used in their work is equivalent to a fractional variability of 0.22 in de Vries et al. (2004) owing to a bias that gets introduced from using integrated flux densities. They also note that the distribution of variability amplitudes seen in their sample is roughly consistent with the extragalactic sample of de Vries et al. (2004).
}

et al. 2014; Keel et al. 2015; LaMassa et al. 2015, for discussions of possibly related phenomena). Assuming a timescale of $\sim 20 \mathrm{yr}$ for an enhanced accretion episode, and given the fact that $50 \mathrm{deg}^{2}$ of the sky has 2000 AGNs with flux density >3 mJy (White et al. 1997; Hopkins et al. 2002), we can estimate the period of occurrence of such episodes over the lifetime of an AGN: $\sim 40,000 \mathrm{yr}$. This is remarkably consistent with previous studies (Reynolds \& Begelman 1997; Czerny et al. 2009; Kunert-Bajraszewska et al. 2010) suggesting some young radio-loud AGNs to have short-lived jets operating on timescales of $10^{4}-10^{5} \mathrm{yr}$.

\subsection{Transient Rates}

We searched our four-epoch data set for transients and found only a single source in the PSC (VTC223612+001006; RSCVn) that was present in one epoch and absent in the rest. Our transient search was carried out over a single-epoch area of $\sim 52 \mathrm{deg}^{2}$, but the sensitivity is not uniform across this area. For the first three epochs, our transient search was conducted on single-pointing images out to a radius $\left(r_{\max }\right)$ of $\sim 8^{\prime}$ from the pointing center. Although the fourth epoch has fairly uniform rms noise, the comparison with the first three epochs for transient search diminishes the significance of the added sensitivity for this epoch. We can approximate the transient rate larger than a threshold flux density, $\kappa(>S)$, using the formulation from Appendix C of Ofek et al. (2011). We use a Gaussian primary beam response with half-width at halfmaximum $\left(r_{\mathrm{HP}}\right)$ of $7 ! 5$ :

$$
\begin{aligned}
\kappa(>S) & =\kappa_{0}\left(S / S_{0}\right)^{-3 / 2} \\
\kappa_{0} & =\frac{3 N_{b} \ln (2)}{2 \pi r_{\mathrm{HP}}^{2}}\left(1-e^{-3 r_{\max }^{2} \ln (2) / r_{\mathrm{HP}}^{2}}\right) .
\end{aligned}
$$

The $2 \sigma$ upper and lower limits for the number of transient events, $N_{b}$, in $50 \mathrm{deg}^{2}$ given that we found one event are 5.683 and $2.3 \times 10^{-2}$ (Gehrels 1986). Since the mean rms noise (flat sky) for the first three epochs of our survey is $70 \mu \mathrm{Jy}$, we use a $7 \sigma$ detection threshold of $0.5 \mathrm{mJy}$. For $\mathrm{RS} \mathrm{CVn}$ variables (active binaries) we calculate a rate of $\kappa(>0.5 \mathrm{mJy})=0.0081$ ${ }_{-0.0079}^{+0.0381}$ events $\mathrm{deg}^{-2}$. For all other types of transients we derive a $95 \%$ confidence upper limit of $\kappa(>0.5 \mathrm{mJy})<0.024$ events $\operatorname{deg}^{-2}$.

The $\log N-\log S$ plots for Galactic and extragalactic slow radio transients are shown in Figure 22. The top panel shows the upper limits to the transient rates ${ }^{31}$ derived from previous radio surveys (colored wedges), the rates derived from radio transient detections (filled circles with errorbars), and the theoretically expected/empirically estimated transient rates (dashed gray lines; see Table 1 for more details). It should be noted that the expected transient rates are not sharp lines but are probability surfaces in the $\log N-\log S$ diagram with the "most probable" rates reported as dashed lines. The dashed line labeled "TDE" represents Swift J1644+57-like Zauderer et al. (2011, 2013) events. Upper limits from a few radio surveys that do not probe any new part of the phase space are not shown in this figure. All observed quantities are color-coded according to the observing frequency. The solid gray line is the rate claimed by Bower et al. (2007), plotted for reference. The upper limit from our pilot survey and the phase space probed by the full CNSS survey are shown as thick green wedges. The phase

\footnotetext{
31 See http://www.tauceti.caltech.edu/kunal/radio-transient-surveys/index. html for more details.
} 

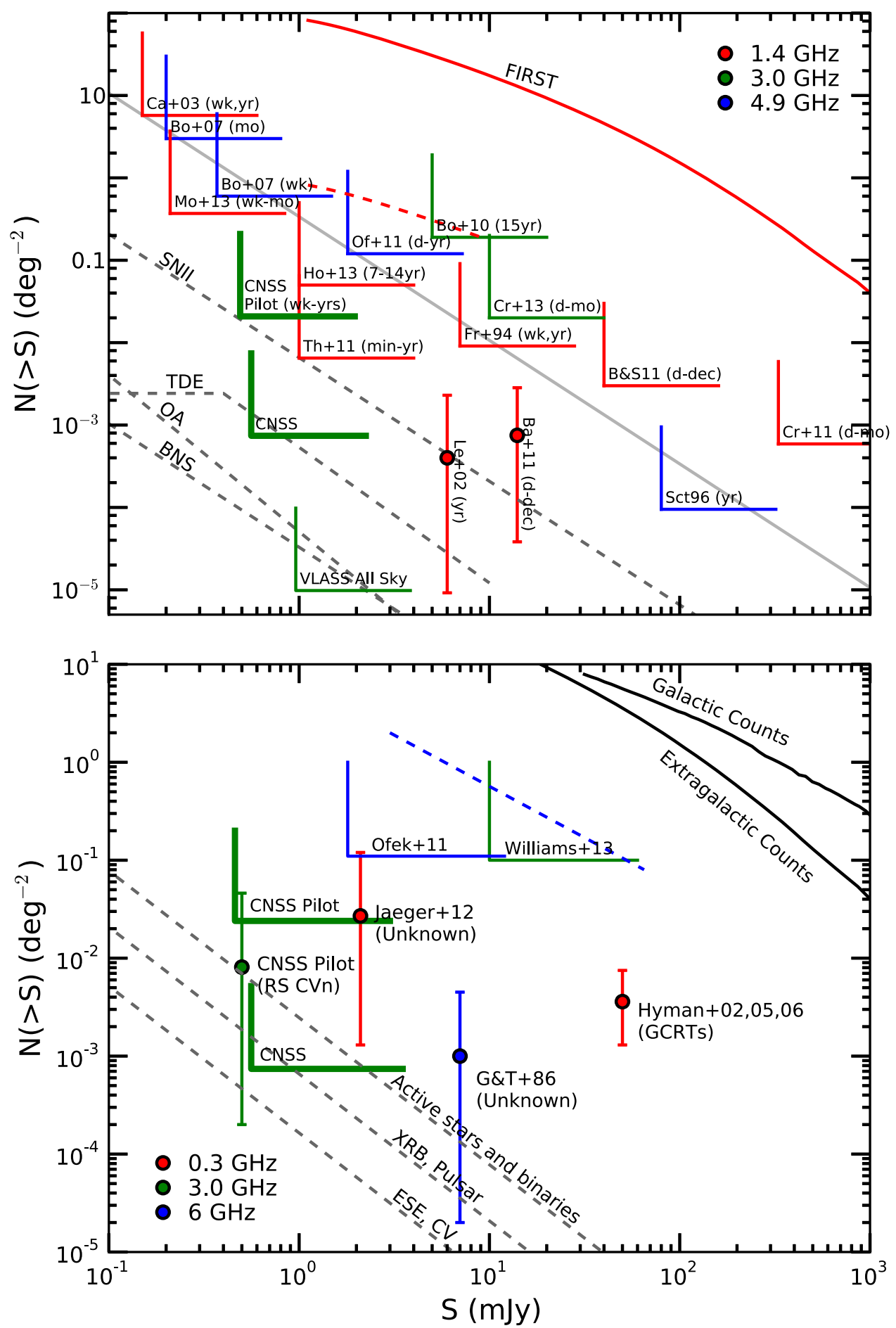

Figure 22. Top: the phase space of slow extragalactic transients. The panel shows the upper limits to the transient rates from previous radio surveys (colored wedges; 95\% confidence), the rates derived from radio transient detections ( $2 \sigma$ error bars), and the expected transient rates. The transient detection labeled as "Le+02" represents an SN II having a peak radio luminosity of $3 \times 10^{27} \mathrm{erg} \mathrm{s}^{-1} \mathrm{~Hz}^{-1}$ and an evolution timescale of $\sim 15 \mathrm{yr}$ (Levinson et al. 2002; Gal-Yam et al. 2006). The one labeled "Ba+11" is a nuclear transient, SUMSS J060938-333508, with a peak radio luminosity of $6 \times 10^{29} \mathrm{erg} \mathrm{s}^{-1} \mathrm{~Hz}^{-1}$ and an evolution timescale of $<5 \mathrm{yr}$ (Bannister et al. 2011a, 2011b, K. Bannister 2015, private communication). All observed quantities are color-coded according to the observing frequency. The solid gray line is the rate claimed by Bower et al. (2007), plotted for reference. The upper limit to the extragalactic transient rate from our pilot survey (this work) and the phase space probed by the full CNSS survey are shown as thick green wedges. The phase space probed by the VLA Sky Survey all-sky tier (VLASS) is also shown. The solid red line denotes the source counts from the FIRST survey, and the dashed red line denotes the approximate counts for strong variables at $1.4 \mathrm{GHz}(1 \%$ of the persistent sources). Bottom: the Galactic transient phase space. Symbols have similar meanings as in the top panel. Black solid lines denote the source counts from the FIRST and the MAGPIS $1.4 \mathrm{GHz}$ surveys. The source counts for variable Galactic sources approximated from Becker et al. (2010) are shown as a blue dashed line. The transient rate for active binaries resulting from our pilot survey is shown by the green error bar, and the upper limit for the rate of all other classes of Galactic transients is denoted by a thick green wedge. See Section 7.2 for more details. 
space probed by the VLA Sky Survey (VLASS) all-sky tier ${ }^{32}$ is shown as a thin green wedge. The source counts from the FIRST survey are represented by the solid red line, and the dashed red line denotes $1 \%$ of these persistent sources, representing strong variable sources at $1.4 \mathrm{GHz}$ (e.g., Mooley et al. 2013, and references therein). Bannister et al. (2011a, 2011b) report only a single extragalactic transient, SUMSS J060938-333508, found to be a nuclear source from ATCA follow-up observations (K. Bannister 2015, private communication). Hence, the transient rate is $7.5 \times 10^{-4}$ events $\mathrm{deg}^{-2}$. Thyagarajan et al. (2011) report 57 transients, but some of these are Galactic and others have indefinite classifications. Hence, we adopt a 95\% confidence level upper limit of 71 transients. The bottom panel of Figure 22 shows the Galactic transient phase space. Symbols have similar meanings as for the extragalactic plot (top panel). For reference, the source counts from the FIRST and the MAGPIS $1.4 \mathrm{GHz}$ (White et al. 2005) surveys are denoted by black solid lines. The approximate source counts for variable Galactic sources from Becker et al. (2010) are denoted by the blue dashed line. The transient rate for active binaries derived from this work is shown by the green error bar, and the upper limit for all other classes of Galactic transients is denoted by a thick green wedge. It is evident from these $\log N-\log S$ diagrams that our pilot survey is not sensitive and wide enough to discover extragalactic explosive transients, but it is already in the regime where stellar flares are expected.

Our contemporaneous optical survey with PTF allows us to make a direct comparison between the transient optical and radio skies. Within the limiting sensitivities of our experiment, the transient skies at these two frequencies appear to be quite distinct. In the 3 months of high-cadence PTF search, eight spectroscopically confirmed supernovae were discovered, none of which were recovered in our radio survey. Conversely, three stellar flares were detected in the radio, but their optical counterparts are saturated in PTF. We thus conclude that future joint radio and optical searches, such as MeerKAT and MeerLICHT, ${ }^{33}$ may only be beneficial if shallow optical surveys are combined with deep radio surveys or vice versa, or if both surveys are deep ( $\mu$ Jy-level sensitivity). We find that deep multiwavelength photometric data and spectroscopy are very important for host and progenitor classification of extragalactic radio transients, and this should inform future slow transient searches. Given the expensive radio observing time, we have attempted to distinguish between AGNs and other transients based on optical light curves from PTF and SDSS. Although in the present study we have optical light curves only for a small fraction of our radio sources (owing to the completeness of optical counterparts), we expect to have $100 \%$ completeness in the LSST era, when radio transient classification based on optical light curves may be a feasible approach.

The radio sky at frequencies of a few $\mathrm{GHz}$ appears to be quiet, with less than a percent of the persistent sources being strong variables. From Table 1 and Figure 22 we find that the rates for a majority of classes of slow radio transients are less than one transient per 10,000 persistent sources. We also find that the fraction of strong variables and explosive transients among persistent sources in the optical sky is similar to the

\footnotetext{
32 See Table 4 of the VLASS proposal for a summary of the all-sky tier, https://safe.nrao.edu/wiki/pub/JVLA/VLASS/VLASS_final.pdf.

33 https://www.astro.ru.nl/wiki/research/meerlicht
}

radio. However, the large number of persistent optical sources per square degree of the sky (down to the limiting sensitivity of PTF, $R=21 \mathrm{mag}$, for example; see Figure 20) implies that the number of varying optical sources is much larger than the number of varying radio sources (down to the limiting sensitivity of our Jansky VLA survey, $\sim 0.5 \mathrm{mJy}$ ). Accordingly, we conclude that the false-positive foreground for future EM$\mathrm{GW}$ searches is lower in the radio than in the optical. ${ }^{34}$

The rates for extragalactic transients, like core-collapse supernovae and BNS mergers, are significantly low (Table 1), and it is not surprising that we found none of these transients in our pilot radio survey. Surveys over large areas of the sky are thus motivated by the search for such exotica. Therefore, we recommend wide-field surveys like that of the entire $\sim 270 \mathrm{deg}^{2}$ of Stripe 82 with the Jansky VLA (the CNSS survey, which is currently under way), all-sky surveys like the VLASS (the allsky tier has been recently approved), or similar surveys with ASKAP, MeerKAT, and WSRT/Apertif. Also, shallow radio surveys are preferred to deep surveys owing to the added advantage of restricting the transient population to a lowredshift space, thus making optical counterpart search feasible.

The authors extend sincere thanks to Joan Wrobel and other scheduling staff at the NRAO in Socorro for extensive help with the scheduling of the VLA observations, and to James Robnett and other computing staff for their untiring assistance with the data storage and allocation of computing resources. The authors also wish to thank Luis Ho, Branimir Sesar, Eran Ofek, Sanjay Bhatnagar, Urvashi Rau, Kumar Golap, Vivek Dhawan, Craig Walker, Talvikki Hovatta, Tim Pearson, Anthony Readhead, Chuck Steidel, and Allison Strom for insightful discussions. The contribution of PTF collaboration members to optical data processing and optical follow-up observations relevant for this project is acknowledged. K.P.M. is grateful to NRAO for the Grote Reber Fellowship, and to Yamini Jangir for going over this manuscript and providing useful suggestions. The National Radio Astronomy Observatory is a facility of the National Science Foundation operated under cooperative agreement by Associated Universities, Inc. S.R.K.'s research in part is supported by NASA and NSF. Some of the data presented herein were obtained at the W. M. Keck Observatory, which is operated as a scientific partnership among the California, Institute of Technology, the University of California and the National Aeronautics and Space Administration. The Observatory was made possible by the generous financial support of the W. M. Keck Foundation. This research has made extensive use of ADS, CDS (Vizier and SIMBAD), NED, SDSS, and IRSA. We thank the anonymous referee for comments that helped in improving certain parts of the manuscript.

\section{APPENDIX}

Given a total observing time $T$, we can do either a wideshallow or a narrow-deep survey, where wide/narrow refers to the area covered, and shallow/deep refers to the sensitivity in terms of limiting flux density. To find which of these will explore the largest volume, consider two surveys, one that

\footnotetext{
34 This statement holds even for optical and radio surveys that have the same limiting flux density, because the number of optical sources per square degree of the sky is expected to be larger than the number of radio sources. Note that the PTF limiting sensitivity of $R=21 \mathrm{mag}$ corresponds to $12 \mu \mathrm{Jy}$.
} 
observes a single pointing for time $T$, and the other that observes $N$ pointings with time $T / N$ alloted for each pointing. We know that the limiting flux density $\left(S_{0}\right)$, integration time $(\tau)$, maximum distance $\left(d_{0}\right)$, and the total volume $\left(V_{0}\right)$ probed by a survey are related as

$$
\begin{gathered}
S_{0} \propto \frac{1}{\sqrt{\tau}} \propto \frac{1}{d_{0}^{2}} \Rightarrow V_{0} \propto d_{0}^{3} \propto \tau^{3 / 4} \\
\therefore \frac{V_{1}}{V_{2}}=\frac{T^{3 / 4}}{N \times(T / N)^{3 / 4}}=\frac{1}{N^{1 / 4}} .
\end{gathered}
$$

Thus, we see that the volume probed by a wide-shallow survey $\left(V_{2}\right)$ is larger than that seen by a narrow-deep survey $\left(V_{1}\right)$ by a factor of the number of pointings to the power of $1 / 4$. However, note that the distance probed in these two kinds of surveys goes as $d_{1} / d_{2}=\sqrt{N}$. This means that if we want to probe transient populations concentrated at large distances (redshifts), it is best to do a narrow-deep survey. Also see Macquart (2014) for survey parameter optimization to maximize the yield of slow transients having well-defined distributions in space.

\section{REFERENCES}

Abazajian, K. N., Adelman-McCarthy, J. K., Agueros, M. A., et al. 2009, ApJS, 182, 543

Aller, M. F., Aller, H. D., Hughes, P. A., et al. 1999, ApJ, 512, 601

Annis, J., Soares-Santos, M., Strauss, M. A., et al. 2014, ApJ, 794, 120

Arshakian, T. G., Leon-Tavares, J., Böttcher, M., et al. 2012, A\&A, 537, 32

Augusteijn, T., Karatasos, K., Papadakis, M., et al. 1992, A\&A, 265, 177

Bannister, K. W., Murphy, T., Gaensler, B. M., Hunstead, R. W., \& Chatterjee, S. 2011a, MNRAS, 412, 634

Bannister, K. W., Murphy, T., Gaensler, B. M., Hunstead, R. W., \& Chatterjee, S. 2011b, MNRAS, 418, 2813

Becker, R. H., Helfand, D. J., White, R. L., \& Proctor, D. D. 2010, ApJ, 140,157

Ben-Ami, S., Gal-Yam, A., Filippenko, A. V., et al. 2012, ApJ, 760, 33

Benz, A. O., \& Güdel, M. 1994, A\&A, 285, 621

Berger, E., Kulkarni, S. R., Frail, D. A., \& Soderberg, A. M. 2003, ApJ, 599, 408

Bevington, P. R., \& Robinson, D. K. 2003, Data Reduction and Error Analysis for the Physical Sciences (3rd ed.; Boston: McGraw-Hill)

Bloom, J. S., Richards, J. W., Nugent, P. E., et al. 2012, PASP, 124, 1175

Boffi, F. R., \& Branch, D. 1995, PASP, 107, 347

Bonzini, M., Mainieri, V., Padovani, P., et al. 2012, ApJS, 203, 15

Booth, R. S., \& Jonas, J. L. 2012, AfrSk, 16, 101

Bourke, S., Mooley, K., \& Hallinan, G. 2014, ASPC, 485, 367

Bower, G. C., Saul, D., Bloom, J. S., et al. 2007, ApJ, 666, 346

Cameron, P. B., Chandra, P., Ray, A., et al. 2005, Natur, 434, 1112

Carilli, C. L., Ivison, R. J., \& Frail, D. A. 2003, ApJ, 590, 192

Cenko, S. B., Krimm, H. A., Horesh, A., et al. 2012, ApJ, 753, 77

Cenko, S. B., Kulkarni, S. R., Horesh, A., et al. 2013, ApJ, 769, 130

Chandra, P., \& Frail, D. A. 2012, ApJ, 746, 156

Chen, X., Rachen, J. P., López-Caniego, M., et al. 2013, A\&A, 553, 107

Chomiuk, L., Krauss, M. I., Rupen, M. P., et al. 2012a, ApJ, 761, 173

Chomiuk, L., Soderberg, A. M., Moe, M., et al. 2012b, ApJ, 750, 164

Condon, J. J., Cotton, W. D., Greisen, E. W., et al. 1998, AJ, 115, 1693

Croft, S., Bower, G. C., Ackermann, R., et al. 2010, ApJ, 719, 45

Cutri, R. M., Wright, E. L., Conrow, T., et al. 2012, http://wise2.ipac.caltech. edu/docs/release/allsky/expsup/index.html

Czerny, B., Siemiginowska, A., Janiuk, A., Nikiel-Wroczyński, B., \& Stawarz, L. 2009, ApJ, 698, 840

Darbha, S., Metzger, B. D., Quataert, E., et al. 2010, MNRAS, 409, 846

Dawson, K. S., Schlegel, D. J., Ahn, C. P., et al. 2013, AJ, 145, 10

de Vries, W. H., Becker, R. H., White, R. L., \& Helfand, D. J. 2004, AJ, 127,2565

Dehnen, W. 1998, AJ, 115, 2384

Dennett-Thorpe, J., \& de Bruyn, A. G. 2002, Natur, 415, 57

Drake, A. J., Djorgovski, S. G., Mahabal, A., et al. 2009, ApJ, 696, 870

El Bouchefry, K., \& Cress, C. M. 2007, AN, 328, 577
Elitzur, M., Ho, L. C., \& Trump, J. R. 2014, MNRAS, 438, 3340 Elvis, M., Wilkes, B. J., McDowell, J. C., et al. 1994, ApJS, 95, 1 Fender, R. P., Muxlow, T. W. B., Garrett, M. A., et al. 2006, MNRAS, 367, 6 Fiedler, R., Dennison, B., Johnston, K. J., et al. 1994, ApJ, 430, 581 Findeisen, K., Hillenbrand, L., Ofek, E., et al. 2013, ApJ, 768, 93 Fong, W., Berger, E., Metzger, B. D., et al. 2014, ApJ, 780, 118 Frail, D. A., Kulkarni, S. R., Hurley, K. C., et al. 1994, ApJ, 437, 43 Frail, D. A., Kulkarni, S. R., Ofek, E. O., Bower, G. C., \& Nakar, E. 2012, ApJ, 747, 70

Franzen, T. M. O., Banfield, J. K., Hales, C. A., et al. 2015, MNRAS, 453, 4020

Fromm, C. M., Perucho, M., Ros, E., et al. 2011, A\&A, 531, 95

Gaensler, B. M., Kouveliotou, C., Gelfand, J. D., et al. 2005, Natur, 434, 1104

Gal-Yam, A., Ofek, E. O., Poznanski, D., et al. 2006, ApJ, 639, 331

Gehrels, N. 1986, ApJ, 303, 336

Gehrels, N., Ramirez-Ruiz, E., Fox, D. B., et al. 2009, ARA\&A, 47, 567

Ghirlanda, G., Burlon, D., Ghisellini, G., et al. 2014, PASA, 31, 22

Güdel, M. 2002, ARA\&A, 40, 217

Hallinan, G., Bourke, S., Lane, C., et al. 2007, ApJ, 663, 25

Hancock, P. J., Gaensler, B. M., \& Murphy, T. 2011, ApJ, 735, 35

Hancock, P. J., Murphy, T., Gaensler, B. M., Hopkins, A., \& Curran, J. R. 2012, MNRAS, 422, 1812

Harding, L. K., Hallinan, G., Boyle, R. P., et al. 2013, ApJ, 779, 101

Henry, G. W., \& Newsom, M. S. 1996, PASP, 108, 242

Hjorth, J., Watson, D., Fynbo, P. U. J., et al. 2005, Natur, 437, 859

Hodge, J. A., Becker, R. H., White, R. L., \& Richards, G. T. 2013, ApJ, 769,125

Hodge, J. A., Becker, R. H., White, R. L., Richards, G. T., \& Zeimann, G. R. 2011, AJ, 142, 3

Hog, E., Fabricius, C., \& Makarov, V. V. 2000, A\&A, 355, 27

Hopkins, A. M., Miller, C. J., Connolly, A. J., et al. 2002, AJ, 123, 1086

Horesh, A., Kulkarni, S. R., Corsi, A., et al. 2013, ApJ, 778, 63

Hovatta, T., Nieppola, E., Tornikoski, M., et al. 2008, A\&A, 485, 51

Huguenin, G. R., Taylor, J. H., Helfand, D. J., et al. 1973, ApJ, 181, 139

Huynh, M. T., Hopkins, A. M., Lenc, E., et al. 2012, PASA, 29, 229

Huynh, M. T., Jackson, C. A., Norris, R. P., Fernandez-Soto, A., et al. 2008, AJ, 135, 2470

Huynh, M. T., Jackson, C. A., Norris, R. P., \& Prandoni, I. 2005, AJ, 130,1373

Hyman, S. D., Lazio, T. J. W., \& Kassim, N. E. 2002, AJ, 123, 1497

Hyman, S. D., Lazio, T. J. W., Kassim, N. E., et al. 2005, Natur, 434, 50

Hyman, S. D., Roy, S., Pal, S., et al. 2007, ApJ, 660, 121

Hyman, S. D., Wijnands, R., Lazio, T. J. W., et al. 2009, ApJ, 696, 280

Ivezic, Z., Menou, K., Knapp, G. R., et al. 2002, AJ, 124, 2364

Johnson, D. R. H., \& Soderblom, D. R. 1987, AJ, 93, 864

Johnston, S., Taylor, R., Bailes, M., et al. 2008, ExA, 22, 151

Kantharia, N. G., Anupama, G. C., Prabhu, T. P., et al. 2007, ApJ, 667, 171

Kasliwal, M. M. 2012, PASA, 29, 482

Keel, W. C., Maksym, P., Bennert, V. N., et al. 2015, AJ, 149, 155

Kesden, M. 2012, PhRvD, 85, 4037

Kharchenko, N. V., \& Roeser, S. 2009, yCat, 1280, 0

Körding, E., Rupen, M., Knigge, C., et al. 2008, Sci, 320, 1318

Kovari, Zs., Vilardell, F., Ribas, I., et al. 2007, AN, 328, 904

Kunert-Bajraszewska, M., Gawroński, M. P., Labiano, A., \& Siemiginowska, A. 2010, MNRAS, 408, 2261

Kunert-Bajraszewska, M., Marecki, A., \& Thomasson, P. 2006, A\&A, 450,945

Laher, R. R., Surace, J., Grillmair, C. J., et al. 2014, PASP, 126, 674

LaMassa, S. M., Cales, S., Moran, E. C., et al. 2015, ApJ, 800, 144

LaMassa, S. M., Urry, C. M., Cappelluti, N., et al. 2013, MNRAS, 436, 3581

Law, N. M., Kulkarni, S. R., Dekany, R. G., et al. 2009, PASP, 121, 1395

Lazio, J. W., Kimball, A., Barger, A. J., et al. 2014, PASP, 126, 196

Levinson, A., Ofek, E. O., Waxman, E., \& Gal-Yam, A. 2002, ApJ, 576, 923

Lister, M. L., Aller, M. F., Aller, H. D., et al. 2013, AJ, 146, 120

Lovell, J. E. J., Rickett, B. J., Macquart, J.-P., et al. 2008, ApJ, 689, 108

Macquart, J.-P. 2014, arXiv:1406.5790

Marscher, A. P., \& Gear, W. K. 1985, ApJ, 298, 114

Mason, B. D., Wycoff, G. L., \& Hartkopf, W. I. 2001, AJ, 122, 3466

McMahon, R. G., White, R. L., Helfand, D. J., \& Becker, R. H. 2002, ApJS, 143,1

Metzger, B. D., Piro, A. L., \& Quataert, E. 2009, MNRAS, 396, 1659

Metzger, B. D., Williams, P. K. G., \& Berger, E. 2015, ApJ, 806, 224

Michelson, P. F., Atwood, W. B., Ritz, S., et al. 2010, RPPh, 73, 7

Miller-Jones, J. C. A., Blundell, K. M., Rupen, M. P., et al. 2004, ApJ, 600,368 
Miller-Jones, J. C. A., Sivakoff, G. R., Altamirano, D., et al. 2012, MNRAS, 421, 468

Mooley, K. P., Frail, D. A., Ofek, E. O., et al. 2013, ApJ, 768, 165

Motch, C., Pakull, M. W., Mouchet, M., et al. 1989, A\&A, 219, 158

Murphy, T., Chatterjee, S., Kaplan, D. L., et al. 2013, PASA, 30, 6

Nakar, E., \& Piran, T. 2011, Natur, 472, 82

Ofek, E. O., Frail, D. A., Breslauer, B., et al. 2011, ApJ, 740, 65

Ofek, E. O., Laher, R., Law, N., et al. 2012, PASP, 124, 62

Oosterloo, T., Verheijen, M., \& van Cappellen, W. 2010, arXiv:1007.5141

Padovani, P., Miller, N., Kellermann, K. I., et al. 2011, ApJ, 740, 20

Panagia, N., Van Dyk, S. D., Weiler, K. W., et al. 2006, ApJ, 646, 369

Patterson, J. 2011, MNRAS, 411, 2695

Perez-Torres, M. A., Lundqvist, P., Beswick, R., et al. 2014, ApJ, 792, 38

Perley, R. A., Chandler, C. J., Butler, B. J., \& Wrobel, J. M. 2011, ApJ, 739, L1

Piran, T., Nakar, E., \& Rosswog, S. 2013, MNRAS, 430, 2121

Piro, A. L., \& Kulkarni, S. R. 2013, ApJL, 762, 17

Polletta, M., Tajer, M., Maraschi, L., et al. 2007, ApJ, 663, 81

Rahmer, G., Smith, R., Velur, V., et al. 2008, Proc. SPIE, 7014, 163

Randall, K. E., Hopkins, A. M., Norris, R. P., et al. 2012, MNRAS, 421, 1644

Rau, A., Kulkarni, S. R., Law, N. M., et al. 2009, PASP, 121, 1334

Reynolds, C. S., \& Begelman, M. C. 1997, ApJ, 487, 135

Richards, J. L., Max-Moerbeck, W., Pavlidou, V., et al. 2011, ApJS, 194, 29

Roy, N., Chomiuk, L., Sokoloski, J. L., et al. 2012, BASI, 40, 293
Smith, A. M., Lynn, S., Sullivan, M., et al. 2011, MNRAS, 412, 1309

Smolcic, V., Schinnerer, E., Scodeggio, M., et al. 2008, ApJS, 177, 14

Soderberg, A. M., Chakraborti, S., Pignata, G., et al. 2010, Natur, 463, 513

Stockdale, C., Weiler, K. W., Panagia, N., et al. 2009, Astro2010: The

Astronomy and Astrophysics Decadal Survey, Science White Papers, no. 288

Student 1908, Biometrika, 6, 1

Tanvir, N. R., Levan, A. J., Fruchter, A. S., et al. 2013, Natur, 500, 547

Teraesranta, H., Tornikoski, M., Mujunen, A., et al. 1998, A\&AS, 132, 305

Thyagarajan, N., Helfand, D. J., White, R. L., \& Becker, R. H. 2011, ApJ, 742, 49

Torres, C. A. O., Quast, G. R., \& da Silva, L. 2006, A\&A, 460, 695

Türler, M., Courvoisier, T. J.-L., \& Paltani, S. 2000, A\&A, 361, 850

Valtaoja, E., Terasranta, H., Urpo, S., et al. 1992, A\&A, 254, 80

Voges, W., Aschenbach, B., \& Boller, T. 1999, A\&A, 349, 389

Weiler, K. W., Panagia, N., Montes, M. J., et al. 2002, ARA\&A, 40, 387

White, R. L., Becker, R. H., \& Helfand, D. J. 2005, ApJ, 130, 586

White, R. L., Becker, R. H., Helfand, D. J., \& Gregg, M. D. 1997, ApJ, 475,479

Williams, P. K. G., Bower, G. C., Croft, S., et al. 2013, ApJ, 762, 85

Woo, J.-K., \& Urry, C. M. 2002, ApJ, 579, 530

Wright, E. L., Eisenhardt, P. R. M., et al. 2010, AJ, 140, 1868

Zauderer, B. A., Berger, E., Margutti, R., et al. 2013, ApJ, 767, 152

Zauderer, B. A., Berger, E., Soderberg, A. M., et al. 2011, Natur, 476, 425 